\title{
Einstein@Home all-sky search for periodic gravitational waves in LIGO S5 data
}

J. Aasi, ${ }^{1}$ J. Abadie, ${ }^{1}$ B. P. Abbott,${ }^{1}$ R. Abbott, ${ }^{1}$ T. D. Abbott,${ }^{2}$ M. Abernathy, ${ }^{3}$ T. Accadia, ${ }^{4}$ F. Acernese,${ }^{5 a, 5 c}$ C. Adams, ${ }^{6}$ T. Adams,${ }^{7}$ P. Addesso, ${ }^{8}$ R. Adhikari, ${ }^{1}$ C. Affeldt, ${ }^{9,10}$ M. Agathos, ${ }^{11 a}$ K. Agatsuma, ${ }^{12}$ P. Ajith, ${ }^{1}$ B. Allen,,${ }^{9,10,13}$ A. Allocca, ${ }^{14 a, 14 c}$ E. Amador Ceron, ${ }^{13}$ D. Amariutei,${ }^{15}$ S. B. Anderson, ${ }^{1}$ W. G. Anderson, ${ }^{13}$ K. Arai, ${ }^{1}$ M. C. Araya, ${ }^{1}$ S. Ast, ${ }^{9,10}$ S. M. Aston, ${ }^{6}$ P. Astone, ${ }^{16 a}$ D. Atkinson, ${ }^{17}$ P. Aufmuth,,${ }^{9,10}$ C. Aulbert, ${ }^{9,10}$ B. E. Aylott,${ }^{18}$ S. Babak, ${ }^{19}$ P. Baker, ${ }^{20}$

G. Ballardin, ${ }^{21}$ S. Ballmer, ${ }^{22}$ Y. Bao,${ }^{15}$ J. C. B. Barayoga,${ }^{1}$ D. Barker,${ }^{17}$ F. Barone,${ }^{5 a, 5 c}$ B. Barr,${ }^{3}$ L. Barsotti, ${ }^{23}$ M. Barsuglia, ${ }^{24}$ M. A. Barton, ${ }^{17}$ I. Bartos, ${ }^{25}$ R. Bassiri, ${ }^{3,26}$ M. Bastarrika, ${ }^{3}$ A. Basti, ${ }^{14 a, 14 b}$ J. Batch, ${ }^{17}$ J. Bauchrowitz, ${ }^{9,10}$ Th. S. Bauer, ${ }^{11 \mathrm{a}}$ M. Bebronne, ${ }^{4}$ D. Beck,${ }^{26}$ B. Behnke, ${ }^{19}$ M. Bejger, ${ }^{27 \mathrm{c}}$ M. G. Beker, ${ }^{11 \mathrm{a}}$ A. S. Bell, ${ }^{3}$ C. Bell, ${ }^{3}$ I. Belopolski, ${ }^{25}$ M. Benacquista, ${ }^{28}$ J. M. Berliner, ${ }^{17}$ A. Bertolini, $,{ }^{9}, 10$ J. Betzwieser, ${ }^{6}$ N. Beveridge, ${ }^{3}$ P. T. Beyersdorf, ${ }^{29}$ T. Bhadbade, ${ }^{26}$ I. A. Bilenko, ${ }^{30}$ G. Billingsley, ${ }^{1}$ J. Birch, ${ }^{6}$ R. Biswas, ${ }^{28}$ M. Bitossi, ${ }^{14 a}$ M. A. Bizouard,${ }^{31 a}$ E. Black, ${ }^{1}$ J. K. Blackburn, ${ }^{1}$ L. Blackburn, ${ }^{32}$ D. Blair, ${ }^{33}$ B. Bland, ${ }^{17}$ M. Blom, ${ }^{11 a}$ O. Bock,${ }^{9,10}$ T. P. Bodiya, ${ }^{23}$ C. Bogan,${ }^{9}{ }^{10}$ C. Bond, ${ }^{18}$ R. Bondarescu, ${ }^{34}$ F. Bondu, ${ }^{35 b}$ L. Bonelli, ${ }^{14 a, 14 b}$ R. Bonnand, ${ }^{36}$ R. Bork, ${ }^{1}$ M. Born, ${ }^{9,10}$ V. Boschi, ${ }^{14 a}$ S. Bose,${ }^{37}$ L. Bosi, ${ }^{38 a}$

B. Bouhou, ${ }^{24}$ S. Braccini, ${ }^{14 a}$ C. Bradaschia, ${ }^{14 a}$ P. R. Brady, ${ }^{13}$ V. B. Braginsky, ${ }^{30}$ M. Branchesi, ${ }^{39 a, 39 b}$ J. E. Brau, ${ }^{40}$ J. Breyer, ${ }^{9,10}$ T. Briant, ${ }^{41}$ D. O. Bridges, ${ }^{6}$ A. Brillet, ${ }^{35 a}$ M. Brinkmann,,${ }^{9,10}$ V. Brisson, ${ }^{31 a}$ M. Britzger, ${ }^{9,10}$ A. F. Brooks, ${ }^{1}$ D. A. Brown, ${ }^{22}$ T. Bulik, ${ }^{27 b}$ H. J. Bulten, ${ }^{11 a, 11 b}$ A. Buonanno, ${ }^{42}$ J. Burguet-Castell, ${ }^{43}$ D. Buskulic, ${ }^{4}$ C. Buy, ${ }^{24}$ R. L. Byer, ${ }^{26}$ L. Cadonati, ${ }^{44}$ G. Cagnoli, ${ }^{36}$ G. Cagnoli, ${ }^{28}$ E. Calloni, ${ }^{5 a, 5 b}$ J. B. Camp,${ }^{32}$ P. Campsie, ${ }^{3}$ K. Cannon, ${ }^{45}$ B. Canuel, ${ }^{21}$ J. Cao, ${ }^{46}$ C. D. Capano, ${ }^{42}$ F. Carbognani, ${ }^{21}$ L. Carbone, ${ }^{18}$ S. Caride,${ }^{47}$ S. Caudill, ${ }^{48}$ M. Cavaglià, ${ }^{49}$ F. Cavalier, ${ }^{31 a}$ R. Cavalieri, ${ }^{21}$ G. Cella, ${ }^{14 a}$ C. Cepeda, ${ }^{1}$ E. Cesarini, ${ }^{39 b}$ T. Chalermsongsak, ${ }^{1}$ P. Charlton,${ }^{50}$ E. Chassande-Mottin, ${ }^{24}$ W. Chen,${ }^{46}$ X. Chen, ${ }^{33}$ Y. Chen, ${ }^{51}$ A. Chincarini, ${ }^{52}$ A. Chiummo, ${ }^{21}$ H. S. Cho, ${ }^{53}$ J. Chow, ${ }^{54}$ N. Christensen, ${ }^{55}$ S. S. Y. Chua, ${ }^{54}$ C. T. Y. Chung, ${ }^{56}$

S. Chung, ${ }^{33}$ G. Ciani, ${ }^{15}$ F. Clara,${ }^{17}$ D. E. Clark,${ }^{26}$ J. A. Clark,${ }^{44}$ J. H. Clayton, ${ }^{13}$ F. Cleva, ${ }^{35 a}$ E. Coccia, ${ }^{57 a, 57 b}$

P.-F. Cohadon, ${ }^{41}$ C. N. Colacino, ${ }^{14 a, 14 b}$ A. Colla,${ }^{16 a, 16 b}$ M. Colombini, ${ }^{16 b}$ A. Conte, ${ }^{16 a, 16 b}$ R. Conte,${ }^{58}$ D. Cook, ${ }^{17}$ T. R. Corbitt, ${ }^{23}$ M. Cordier, ${ }^{29}$ N. Cornish, ${ }^{20}$ A. Corsi, ${ }^{1}$ C. A. Costa ${ }^{48,59}$ M. Coughlin, ${ }^{55}$ J.-P. Coulon, ${ }^{35 a}$ P. Couvares, ${ }^{22}$ D. M. Coward ${ }^{33}$ M. Cowart, ${ }^{6}$ D. C. Coyne, ${ }^{1}$ J. D. E. Creighton, ${ }^{13}$ T. D. Creighton, ${ }^{28}$ A. M. Cruise, ${ }^{18}$ A. Cumming, ${ }^{3}$ L. Cunningham, ${ }^{3}$ E. Cuoco, ${ }^{21}$ R. M. Cutler, ${ }^{18}$ K. Dahl,,${ }^{9,10}$ M. Damjanic, ${ }^{9,10}$ S. L. Danilishin, ${ }^{33}$ S. D'Antonio, ${ }^{57 a}$ K. Danzmann, ${ }^{9,10}$ V. Dattilo, ${ }^{21}$ B. Daudert, ${ }^{1}$ H. Daveloza,${ }^{28}$ M. Davier, ${ }^{31 a}$ E. J. Daw,${ }^{60}$ R. Day, ${ }^{21}$ T. Dayanga,${ }^{37}$ R. De Rosa ${ }^{5,5 b}$ D. DeBra ${ }^{26}$ G. Debreczeni,${ }^{61}$ J. Degallaix, ${ }^{36}$ W. Del Pozzo,${ }^{11 a}$ T. Dent, ${ }^{7}$ V. Dergachev, ${ }^{1}$ R. DeRosa, ${ }^{48}$ S. Dhurandhar, ${ }^{62}$ L. Di Fiore, ${ }^{5 a}$ A. Di Lieto, ${ }^{14 a}, 14 \mathrm{~b}$ I. Di Palma, ${ }^{9,10}$ M. Di Paolo Emilio, ${ }^{57 a, 57 c}$ A. Di Virgilio, ${ }^{14 a}$ M. Díaz, ${ }^{28}$ A. Dietz, ${ }^{49}$ A. Dietz,${ }^{4}$ F. Donovan, ${ }^{23}$ K. L. Dooley, ${ }^{9}{ }^{10}$ S. Doravari, ${ }^{1}$ S. Dorsher, ${ }^{63}$ M. Drago, ${ }^{64 a, 64 b}$ R. W. P. Drever, ${ }^{65}$ J. C. Driggers, ${ }^{1}$ Z. Du, ${ }^{46}$ J.-C. Dumas,${ }^{33}$ S. Dwyer ${ }^{23}$ T. Eberle, ${ }^{9,10}$ M. Edgar, ${ }^{3}$ M. Edwards, ${ }^{7}$ A. Effler, ${ }^{48}$ P. Ehrens, ${ }^{1}$ G. Endrőczi ${ }^{61}$ R. Engel,,${ }^{1}$ T. Etzel, ${ }^{1}$ K. Evans,${ }^{3}$ M. Evans,${ }^{23}$ T. Evans,${ }^{6}$ M. Factourovich ${ }^{25}$ V. Fafone,${ }^{57 a, 57 b}$ S. Fairhurst, ${ }^{7}$ B. F. Farr, ${ }^{66}$ M. Favata, ${ }^{13}$ D. Fazi, ${ }^{66}$ H. Fehrmann, ${ }^{96}$ D. Feldbaum, ${ }^{15}$ I. Ferrante,,${ }^{14 a, 14 b}$ F. Ferrini, ${ }^{21}$ F. Fidecaro, ${ }^{14 a, 14 b}$ L. S. Finn,${ }^{34}$ I. Fiori, ${ }^{21}$ R. P. Fisher, ${ }^{22}$ R. Flaminio, ${ }^{36}$ S. Foley, ${ }^{23}$ E. Forsi, ${ }^{6}$ N. Fotopoulos, ${ }^{1}$ J.-D. Fournier, ${ }^{35 a}$ J. Franc, ${ }^{36}$ S. Franco, ${ }^{31 \mathrm{a}}$ S. Frasca, ${ }^{16 a, 16 \mathrm{~b}}$ F. Frasconi, ${ }^{14 \mathrm{a}}$ M. Frede, ${ }^{9,10}$ M. A. Frei, ${ }^{67}$ Z. Frei ${ }^{68}$ A. Freise,${ }^{18}$ R. Frey, ${ }^{40}$ T. T. Fricke,,${ }^{9,10}$ D. Friedrich, ${ }^{9}, 10$ P. Fritschel, ${ }^{23}$ V. V. Frolov, ${ }^{6}$ M.-K. Fujimoto, ${ }^{12}$ P. J. Fulda, ${ }^{18}$ M. Fyffe, ${ }^{6}$ J. Gair, ${ }^{69}$ M. Galimberti, ${ }^{36}$ L. Gammaitoni ${ }^{38 a, 38 b}$ J. Garcia, ${ }^{17}$ F. Garufi, ${ }^{5 a, 5 b}$ M. E. Gáspár, ${ }^{61}$ G. Gelencser, ${ }^{68}$ G. Gemme, ${ }^{52}$ E. Genin,${ }^{21}$ A. Gennai, ${ }^{14 a}$ L. Á. Gergely, ${ }^{70}$ S. Ghosh, ${ }^{37}$ J. A. Giaime,,${ }^{6,4}$ S. Giampanis, ${ }^{13}$ K. D. Giardina,${ }^{6}$ A. Giazotto, ${ }^{14 a}$ S. Gil-Casanova, ${ }^{43}$ C. Gill, ${ }^{3}$ J. Gleason, ${ }^{15}$ E. Goetz, ${ }^{9,10}$ G. González, ${ }^{48}$ M. L. Gorodetsky, ${ }^{30}$ S. Goßler, ${ }^{9,10}$ R. Gouaty, ${ }^{4}$ C. Graef, ${ }^{9,10}$ P. B. Graff, ${ }^{36}$ M. Granata, ${ }^{36}$ A. Grant, ${ }^{3}$ C. Gray, ${ }^{17}$ R. J. S. Greenhalgh, ${ }^{71}$ A. M. Gretarsson, ${ }^{72}$ C. Griffo, ${ }^{2}$ H. Grote,,${ }^{9,10}$ K. Grover, ${ }^{18}$

S. Grunewald, ${ }^{19}$ G. M. Guidi, ${ }^{39 a, 39 b}$ C. Guido, ${ }^{6}$ R. Gupta, ${ }^{62}$ E. K. Gustafson, ${ }^{1}$ R. Gustafson, ${ }^{47}$ J. M. Hallam, ${ }^{18}$ D. Hammer, ${ }^{13}$ G. Hammond, ${ }^{3}$ J. Hanks,${ }^{17}$ C. Hanna, ${ }^{1,73}$ J. Hanson, ${ }^{6}$ J. Harms,${ }^{65}$ G. M. Harry, ${ }^{74}$ I. W. Harry, ${ }^{22}$ E. D. Harstad, ${ }^{40}$ M. T. Hartman, ${ }^{15}$ K. Haughian, ${ }^{3}$ K. Hayama, ${ }^{12}$ J.-F. Hayau, ${ }^{35 b}$ J. Heefner, ${ }^{1}$ A. Heidmann, ${ }^{41}$ H. Heitmann, ${ }^{35 a}$ P. Hello, ${ }^{31 a}$ M. A. Hendry, ${ }^{3}$ I. S. Heng, ${ }^{3}$ A. W. Heptonstall, ${ }^{1}$ V. Herrera, ${ }^{26}$ M. Heurs,,${ }^{9} 10$ M. Hewitson, ${ }^{9,10}$ S. Hild ${ }^{3}$ D. Hoak ${ }^{44}$ K. A. Hodge, ${ }^{1}$ K. Holt,${ }^{6}$ M. Holtrop,${ }^{75}$ T. Hong ${ }^{51}$ S. Hooper, ${ }^{33}$ J. Hough, ${ }^{3}$ E. J. Howell, ${ }^{33}$ B. Hughey, ${ }^{13}$ S. Husa, ${ }^{43}$ S. H. Huttner, ${ }^{3}$ T. Huynh-Dinh,,${ }^{6}$ D. R. Ingram,${ }^{17}$ R. Inta, ${ }^{54}$ T. Isogai, ${ }^{55}$ A. Ivanov, ${ }^{1}$ K. Izumi, ${ }^{12}$ M. Jacobson, ${ }^{1}$ E. James, ${ }^{1}$ Y. J. Jang, ${ }^{43}$ P. Jaranowski, ${ }^{27 d}$ E. Jesse, ${ }^{72}$ W. W. Johnson, ${ }^{48}$ D. I. Jones, ${ }^{76}$ R. Jones, ${ }^{3}$

R. J. G. Jonker, ${ }^{11 a}$ L. Ju, ${ }^{33}$ P. Kalmus, ${ }^{1}$ V. Kalogera, ${ }^{66}$ S. Kandhasamy, ${ }^{63}$ G. Kang, ${ }^{77}$ J. B. Kanner, ${ }^{32,42}$ M. Kasprzack,${ }^{21,31 a}$ R. Kasturi, ${ }^{78}$ E. Katsavounidis, ${ }^{23}$ W. Katzman, ${ }^{6}$ H. Kaufer,,${ }^{9} 10$ K. Kaufman, ${ }^{51}$ K. Kawabe, ${ }^{17}$ S. Kawamura, ${ }^{12}$

F. Kawazoe, ${ }^{9,10}$ D. Keitel, ${ }^{9,10}$ D. Kelley, ${ }^{22}$ W. Kells, ${ }^{1}$ D. G. Keppel, ${ }^{1}$ Z. Keresztes, ${ }^{70}$ A. Khalaidovski, ${ }^{9,10}$ F. Y. Khalili, ${ }^{30}$ E. A. Khazanov, ${ }^{79}$ B. K. Kim, ${ }^{77}$ C. Kim, ${ }^{80}$ H. Kim, ${ }^{9}, 10$ K. Kim, ${ }^{81}$ N. Kim, ${ }^{26}$ Y. M. Kim,${ }^{53}$ P. J. King, ${ }^{1}$ D. L. Kinzel, ${ }^{6}$ J. S. Kissel, ${ }^{23}$ S. Klimenko, ${ }^{15}$ J. Kline, ${ }^{13}$ K. Kokeyama, ${ }^{48}$ V. Kondrashov, ${ }^{1}$ S. Koranda, ${ }^{13}$ W. Z. Korth, ${ }^{1}$ I. Kowalska, ${ }^{27 b}$ 
D. Kozak, ${ }^{1}$ V. Kringel,${ }^{9,10}$ B. Krishnan, ${ }^{19}$ A. Królak, ${ }^{27 a, 27 e}$ G. Kuehn, ${ }^{9,10}$ P. Kumar, ${ }^{22}$ R. Kumar, ${ }^{3}$ R. Kurdyumov, ${ }^{26}$ P. Kwee, ${ }^{23}$ P. K. Lam, ${ }^{54}$ M. Landry, ${ }^{17}$ A. Langley,${ }^{65}$ B. Lantz,${ }^{26}$ N. Lastzka, ${ }^{9,10}$ C. Lawrie, ${ }^{3}$ A. Lazzarini, ${ }^{1}$ P. Leaci, ${ }^{19}$ C. H. Lee ${ }^{53}$ H. K. Lee, ${ }^{81}$ H. M. Lee ${ }^{82}$ J. R. Leong,,${ }^{910}$ I. Leonor, ${ }^{40}$ N. Leroy,${ }^{31 a}$ N. Letendre, ${ }^{4}$ V. Lhuillier,${ }^{17}$ J. Li, ${ }^{46}$ T. G. F. Li ${ }^{11 \text { a }}$ P. E. Lindquist, ${ }^{1}$ V. Litvine, ${ }^{1}$ Y. Liu ${ }^{46}$ Z. Liu, ${ }^{15}$ N. A. Lockerbie,${ }^{83}$ D. Lodhia, ${ }^{18}$ J. Logue,${ }^{3}$ M. Lorenzini, ${ }^{39 a}$ V. Loriette, ${ }^{31 \mathrm{~b}}$ M. Lormand, ${ }^{6}$ G. Losurdo, ${ }^{39 \mathrm{a}}$ J. Lough, ${ }^{22}$ M. Lubinski, ${ }^{17}$ H. Lück,,${ }^{9,10}$ A. P. Lundgren, ${ }^{9,10}$ J. Macarthur, ${ }^{3}$ E. Macdonald, ${ }^{3}$ B. Machenschalk, ${ }^{9,10}$ M. MacInnis, ${ }^{23}$ D. M. Macleod, ${ }^{7}$ M. Mageswaran, ${ }^{1}$ K. Mailand, ${ }^{1}$ E. Majorana, ${ }^{16 a}$ I. Maksimovic, ${ }^{31 b}$ V. Malvezzi, ${ }^{57 a}$ N. Man, ${ }^{35 a}$ I. Mandel,${ }^{18}$ V. Mandic, ${ }^{63}$ M. Mantovani, ${ }^{14 a, 14 c}$ F. Marchesoni, ${ }^{38 a}$ F. Marion, ${ }^{4}$ S. Márka, ${ }^{25}$ Z. Márka, ${ }^{25}$ A. Markosyan, ${ }^{26}$ E. Maros, ${ }^{1}$ J. Marque, ${ }^{21}$ F. Martelli,,${ }^{39 a, 39 b}$ I. W. Martin, ${ }^{3}$ R. M. Martin, ${ }^{15}$ J. N. Marx, ${ }^{1}$ K. Mason, ${ }^{23}$ A. Masserot, ${ }^{4}$ F. Matichard ${ }^{23}$ L. Matone, ${ }^{25}$ R. A. Matzner, ${ }^{84}$ N. Mavalvala,${ }^{23}$ G. Mazzolo, ${ }^{9,10}$ R. McCarthy, ${ }^{17}$ D. E. McClelland,${ }^{54}$ S. C. McGuire,${ }^{85}$ G. McIntyre, ${ }^{1}$ J. McIver, ${ }^{44}$ G. D. Meadors ${ }^{47}$ M. Mehmet,${ }^{9,10}$ T. Meier, ${ }^{9,10}$ A. Melatos,${ }^{56}$ A. C. Melissinos, ${ }^{86}$ G. Mendell,${ }^{17}$ D. F. Menéndez,${ }^{34}$ R. A. Mercer, ${ }^{13}$ S. Meshkov, ${ }^{1}$ C. Messenger, ${ }^{7}$ M. S. Meyer, ${ }^{6}$ H. Miao,${ }^{51}$ C. Michel,${ }^{36}$ L. Milano,${ }^{5 a, 5 b}$ J. Miller,${ }^{54}$ Y. Minenkov, ${ }^{57 a}$ C. M. F. Mingarelli, ${ }^{18}$ V. P. Mitrofanov,${ }^{30}$ G. Mitselmakher,${ }^{15}$ R. Mittleman,${ }^{23}$ B. Moe, ${ }^{13}$ M. Mohan, ${ }^{21}$

S. R. P. Mohapatra, ${ }^{44}$ D. Moraru, ${ }^{17}$ G. Moreno, ${ }^{17}$ N. Morgado, ${ }^{36}$ A. Morgia, ${ }^{57 a, 57 b}$ T. Mori, ${ }^{12}$ S. R. Morriss, ${ }^{28}$ S. Mosca, ${ }^{5 a, 5 b}$ K. Mossavi, ${ }^{9,10}$ B. Mours, ${ }^{4}$ C. M. Mow-Lowry, ${ }^{54}$ C. L. Mueller ${ }^{15}$ G. Mueller,${ }^{15}$ S. Mukherjee,${ }^{28}$ A. Mullavey, ${ }^{48,54}$ H. Müller-Ebhardt, ${ }^{9,10}$ J. Munch, ${ }^{87}$ D. Murphy, ${ }^{25}$ P. G. Murray, ${ }^{3}$ A. Mytidis, ${ }^{15}$ T. Nash, ${ }^{1}$ L. Naticchioni, ${ }^{16 a, 16 b}$ V. Necula, ${ }^{15}$ J. Nelson, ${ }^{3}$ I. Neri, ${ }^{38 a, 38 b}$ G. Newton, ${ }^{3}$ T. Nguyen, ${ }^{54}$ A. Nishizawa, ${ }^{12}$ A. Nitz, ${ }^{22}$ F. Nocera, ${ }^{21}$ D. Nolting, ${ }^{6}$ M. E. Normandin, ${ }^{28}$ L. Nuttall, ${ }^{7}$ E. Ochsner, ${ }^{13}$ J. O'Dell, ${ }^{71}$ E. Oelker, ${ }^{23}$ G. H. Ogin, ${ }^{1}$ J. J. Oh, ${ }^{88}$ S. H. Oh, ${ }^{88}$ R. G. Oldenberg, ${ }^{13}$ B. O'Reilly, ${ }^{6}$ R. O'Shaughnessy, ${ }^{13}$ C. Osthelder, ${ }^{1}$ C. D. Ott, ${ }^{51}$ D. J. Ottaway, ${ }^{87}$ R. S. Ottens,${ }^{15}$ H. Overmier, ${ }^{6}$ B. J. Owen, ${ }^{34}$ A. Page,${ }^{18}$ L. Palladino, ${ }^{57 a, 57 c}$ C. Palomba, ${ }^{16 a}$ Y. Pan, ${ }^{42}$ F. Paoletti, ${ }^{14 a, 21}$ R. Paoletti, ${ }^{14 a}$ M. A. Papa,${ }^{13,19}$ M. Parisi,${ }^{5,5 b}$ A. Pasqualetti, ${ }^{21}$ R. Passaquieti, ${ }^{14 a, 14 b}$ D. Passuello, ${ }^{14 \mathrm{a}}$ M. Pedraza, ${ }^{1}$ S. Penn,${ }^{78}$ A. Perreca, ${ }^{22}$ G. Persichetti, ${ }^{5 a, 5 b}$ M. Phelps,${ }^{1}$ M. Pichot,${ }^{35 a}$ M. Pickenpack,,${ }^{9,10}$ F. Piergiovanni,${ }^{39 a, 39 b}$ V. Pierro, ${ }^{8}$ M. Pihlaja ${ }^{63}$ L. Pinard,${ }^{36}$ I. M. Pinto, ${ }^{8}$ M. Pitkin, ${ }^{3}$ H. J. Pletsch,,${ }^{9,10}$ M. V. Plissi, ${ }^{3}$ R. Poggiani, ${ }^{14 a, 14 b}$ J. Pöld, ${ }^{9,10}$ F. Postiglione,${ }^{58}$ C. Poux,${ }^{1}$ M. Prato,${ }^{52}$ V. Predoi, ${ }^{7}$ T. Prestegard,${ }^{63}$ L. R. Price, ${ }^{1}$ M. Prijatelj, ${ }^{9,10}$ M. Principe,${ }^{8}$ S. Privitera, ${ }^{1}$ R. Prix,${ }^{9,10}$ G. A. Prodi, ${ }^{64 a, 64 b}$ L. G. Prokhorov, ${ }^{30}$ O. Puncken,,${ }^{9,10}$ M. Punturo, ${ }^{38 a}$ P. Puppo, ${ }^{16 a}$ V. Quetschke, ${ }^{28}$ R. Quitzow-James, ${ }^{40}$ F. J. Raab, ${ }^{17}$ D. S. Rabeling, ${ }^{11 \mathrm{a}, 11 \mathrm{~b}}$ I. Rácz, ${ }^{61}$ H. Radkins, ${ }^{17}$ P. Raffai, ${ }^{25,68}$ M. Rakhmanov, ${ }^{28}$ C. Ramet, ${ }^{6}$ B. Rankins,${ }^{49}$ P. Rapagnani, ${ }^{16 a, 16 b}$ V. Raymond,${ }^{66}$ V. Re,${ }^{57 a, 57 b}$ C. M. Reed,${ }^{17}$ T. Reed,${ }^{89}$ T. Regimbau, ${ }^{35 a}$ S. Reid, ${ }^{3}$ D. H. Reitze, ${ }^{1}$ F. Ricci, ${ }^{16 a, 16 b}$ R. Riesen, ${ }^{6}$ K. Riles, ${ }^{47}$ M. Roberts, ${ }^{26}$ N. A. Robertson,,${ }^{1,3}$ F. Robinet, ${ }^{31 \mathrm{a}}$ C. Robinson, ${ }^{7}$ E. L. Robinson, ${ }^{19}$ A. Rocchi, ${ }^{57 a}$ S. Roddy, ${ }^{6}$ C. Rodriguez, ${ }^{66}$ M. Rodruck, ${ }^{17}$ L. Rolland, ${ }^{4}$ J. G. Rollins, ${ }^{1}$ J. D. Romano, ${ }^{28}$ R. Romano, ${ }^{5 a, 5 \mathrm{c}}$ J. H. Romie, ${ }^{6}$ D. Rosińska, ${ }^{27 c, 27 f}$ C. Röver, ${ }^{9,10}$ S. Rowan, ${ }^{3}$ A. Rüdiger,${ }^{9,10}$ P. Ruggi, ${ }^{21}$ K. Ryan, ${ }^{17}$ F. Salemi, ${ }^{9,10}$ L. Sammut,${ }^{56}$ V. Sandberg, ${ }^{17}$ S. Sankar, ${ }^{23}$ V. Sannibale, ${ }^{1}$ L. Santamaría, ${ }^{1}$ I. Santiago-Prieto, ${ }^{3}$ G. Santostasi, ${ }^{90}$ E. Saracco, ${ }^{36}$ B. S. Sathyaprakash, ${ }^{7}$ P. R. Saulson, ${ }^{22}$ R. L. Savage, ${ }^{17}$ R. Schilling, ${ }^{9,10}$ R. Schnabel, ${ }^{9,10}$ R. M. S. Schofield, ${ }^{40}$ B. Schulz, ${ }^{9,10}$ B. F. Schutz, ${ }^{7,19}$ P. Schwinberg, ${ }^{17}$ J. Scott, ${ }^{3}$ S. M. Scott, ${ }^{54}$ F. Seifert, ${ }^{1}$ D. Sellers, ${ }^{6}$ D. Sentenac, ${ }^{21}$ A. Sergeev, ${ }^{79}$ D. A. Shaddock, ${ }^{54}$ M. Shaltev, ${ }^{9,10}$ B. Shapiro, ${ }^{23}$ P. Shawhan, ${ }^{42}$ D. H. Shoemaker, ${ }^{23}$ T. L. Sidery, ${ }^{18}$ X. Siemens, ${ }^{13}$ D. Sigg, ${ }^{17}$ D. Simakov, ${ }^{9,10}$ A. Singer, ${ }^{1}$ L. Singer, ${ }^{1}$ A. M. Sintes,${ }^{43}$ G. R. Skelton, ${ }^{13}$ B. J. J. Slagmolen, ${ }^{54}$ J. Slutsky, ${ }^{4}$ J. R. Smith, ${ }^{2}$ M. R. Smith, ${ }^{1}$ R. J. E. Smith, ${ }^{18}$ N. D. Smith-Lefebvre, ${ }^{15}$ K. Somiya, ${ }^{51}$ B. Sorazu, ${ }^{3}$ F. C. Speirits, ${ }^{3}$ L. Sperandio, ${ }^{57 a, 57 b}$ M. Stefszky, ${ }^{54}$ E. Steinert, ${ }^{17}$ J. Steinlechner, ${ }^{9,10}$ S. Steinlechner, ${ }^{9,10}$ S. Steplewski, ${ }^{37}$ A. Stochino, ${ }^{1}$ R. Stone,${ }^{28}$ K. A. Strain, ${ }^{3}$ S. E. Strigin,${ }^{30}$ A. S. Stroeer, ${ }^{28}$ R. Sturani, ${ }^{39 a, 39 b}$ A. L. Stuver, ${ }^{6}$ T.Z. Summerscales, ${ }^{91}$ M. Sung, ${ }^{48}$ S. Susmithan, ${ }^{33}$ P. J. Sutton, ${ }^{7}$ B. Swinkels, ${ }^{21}$ G. Szeifert,${ }^{68}$ M. Tacca, ${ }^{21}$ L. Taffarello, ${ }^{64 c}$

D. Talukder ${ }^{37}$ D. B. Tanner, ${ }^{15}$ S. P. Tarabrin,,${ }^{9} 10$ R. Taylor, ${ }^{1}$ A. P. M. ter Braack, ${ }^{11 a}$ P. Thomas, ${ }^{17}$ K. A. Thorne,${ }^{6}$

K. S. Thorne, ${ }^{51}$ E. Thrane, ${ }^{63}$ A. Thüring, ${ }^{9,10}$ C. Titsler, ${ }^{34}$ K. V. Tokmakov ${ }^{83}$ C. Tomlinson, ${ }^{60}$ A. Toncelli, ${ }^{14 a, 14 b}$ M. Tonelli, ${ }^{14 \mathrm{a}, 14 \mathrm{~b}}$ O. Torre, ${ }^{14 \mathrm{a}, 14 \mathrm{c}}$ C. V. Torres, ${ }^{28}$ C. I. Torrie, ${ }^{1,3}$ E. Tournefier, ${ }^{4}$ F. Travasso, ${ }^{38 \mathrm{a}, 38 \mathrm{~b}}$ G. Traylor, ${ }^{6} \mathrm{M}$. Tse, ${ }^{25}$ D. Ugolini,${ }^{92}$ H. Vahlbruch, ${ }^{9,10}$ G. Vajente, ${ }^{14 a, 14 b}$ J. F. J. van den Brand, ${ }^{11 a, 11 b}$ C. Van Den Broeck, ${ }^{11 \mathrm{a}}$ S. van der Putten, ${ }^{11 \mathrm{a}}$ A. A. van Veggel, ${ }^{3}$ S. Vass, ${ }^{1}$ M. Vasuth, ${ }^{61}$ R. Vaulin, ${ }^{23}$ M. Vavoulidis, ${ }^{31 \mathrm{a}}$ A. Vecchio, ${ }^{18}$ G. Vedovato, ${ }^{64 \mathrm{c}} \mathrm{J}$. Veitch, ${ }^{7}$ P. J. Veitch ${ }^{87}$ K. Venkateswara, ${ }^{93}$ D. Verkindt, ${ }^{4}$ F. Vetrano, ${ }^{39 a, 39 b}$ A. Viceré, ${ }^{39 a, 39 b}$ A. E. Villar, ${ }^{1}$ J.-Y. Vinet, ${ }^{35 a}$ S. Vitale, ${ }^{11 a}$ H. Vocca, ${ }^{38 a}$ C. Vorvick,${ }^{17}$ S. P. Vyatchanin, ${ }^{30}$ A. Wade,${ }^{54}$ L. Wade,${ }^{13}$ M. Wade,${ }^{13}$ S. J. Waldman, ${ }^{23}$ L. Wallace, ${ }^{1}$ Y. Wan,${ }^{46}$ M. Wang, ${ }^{18}$ X. Wang, ${ }^{46}$ A. Wanner, ${ }^{9,10}$ R. L. Ward, ${ }^{24}$ M. Was, ${ }^{31 a}$ M. Weinert, ${ }^{9,10}$ A. J. Weinstein, ${ }^{1}$ R. Weiss, ${ }^{23}$ T. Welborn, ${ }^{6}$ L. Wen, ${ }^{33,51}$ P. Wessels, ${ }^{9,10}$ M. West, ${ }^{22}$ T. Westphal, ${ }^{9,10}$ K. Wette,,${ }^{9,10}$ J. T. Whelan, ${ }^{67}$ S. E. Whitcomb, ${ }^{1,33}$ D. J. White, ${ }^{60}$ B. F. Whiting, ${ }^{15}$ K. Wiesner,,${ }^{9,10}$ C. Wilkinson, ${ }^{17}$ P. A. Willems, ${ }^{1}$ L. Williams,${ }^{15}$ R. Williams, ${ }^{1}$ B. Willke, ${ }^{9,10}$ M. Wimmer, ${ }^{9,10}$ L. Winkelmann, ${ }^{9,10}$ W. Winkler, ${ }^{9,10}$ C. C. Wipf, ${ }^{23}$ A. G. Wiseman, ${ }^{13}$ H. Wittel,,${ }^{9,10}$ G. Woan, ${ }^{3}$ R. Wooley, ${ }^{6}$ J. Worden, ${ }^{17}$ J. Yablon, ${ }^{66}$ I. Yakushin, ${ }^{6}$ H. Yamamoto, ${ }^{1}$ K. Yamamoto, ${ }^{64 b, 64 d}$ C. C. Yancey, ${ }^{42}$ H. Yang, ${ }^{51}$ 
D. Yeaton-Massey, ${ }^{1}$ S. Yoshida, ${ }^{94}$ M. Yvert, ${ }^{4}$ A. Zadrożny, ${ }^{27 e}$ M. Zanolin, ${ }^{72}$ J.-P. Zendri, ${ }^{64 c}$ F. Zhang, ${ }^{46}$ L. Zhang, ${ }^{1}$ C. Zhao, ${ }^{33}$ N. Zotov, ${ }^{89}$ M. E. Zucker, ${ }^{23}$ J. Zweizig, ${ }^{1}$ and D. P. Anderson ${ }^{95}$

(The LIGO Scientific Collaboration and the Virgo Collaboration)

${ }^{1}$ LIGO-California Institute of Technology, Pasadena, California 91125, USA

${ }^{2}$ California State University Fullerton, Fullerton, California 92831, USA

${ }^{3}$ SUPA, University of Glasgow, Glasgow, G12 8QQ, United Kingdom

${ }^{4}$ Laboratoire d'Annecy-le-Vieux de Physique des Particules (LAPP), Université de Savoie, CNRS/IN2P3, F-74941 Annecy-Le-Vieux, France

${ }^{5 a}$ INFN-Sezione di Napoli, I-80126 Napoli, Italy

${ }^{5 \mathrm{~b}}$ Università di Napoli 'Federico II', Complesso Universitario di Monte S. Angelo, I-80126 Napoli, Italy

${ }^{5 \mathrm{c}}$ Università di Salerno, Fisciano, I-84084 Salerno, Italy

${ }^{6}$ LIGO-Livingston Observatory, Livingston, Louisiana 70754, USA

${ }^{7}$ Cardiff University, Cardiff CF24 3AA, United Kingdom

${ }^{8}$ University of Sannio at Benevento, I-82100 Benevento, Italy and INFN (Sezione di Napoli), I-80126 Napoli, Italy

${ }^{9}$ Albert-Einstein-Institut, Max-Planck-Institut für Gravitationsphysik, D-30167 Hannover, Germany

${ }^{10}$ Leibniz Universität Hannover, D-30167 Hannover, Germany

${ }^{11 a}$ Nikhef, Science Park, Amsterdam, The Netherlands

${ }^{11 \mathrm{~b}}$ VU University Amsterdam, De Boelelaan 1081, 1081 HV Amsterdam, The Netherlands

${ }^{12}$ National Astronomical Observatory of Japan, Tokyo 181-8588, Japan

${ }^{13}$ University of Wisconsin-Milwaukee, Milwaukee, Wisconsin 53201, USA

${ }^{14 a}$ INFN, Sezione di Pisa, I-56127 Pisa, Italy

${ }^{14 \mathrm{~b}}$ Università di Pisa, I-56127 Pisa, Italy

${ }^{14 \mathrm{c}}$ Università di Siena, I-53100 Siena, Italy

${ }^{15}$ University of Florida, Gainesville, Florida 32611, USA

${ }^{16 a}$ INFN, Sezione di Roma, I-00185 Roma, Italy

${ }^{16 \mathrm{~b}}$ Università 'La Sapienza', I-00185 Roma, Italy

${ }^{17}$ LIGO-Hanford Observatory, Richland, Washington 99352, USA

${ }^{18}$ University of Birmingham, Birmingham B15 2TT, United Kingdom

${ }^{19}$ Albert-Einstein-Institut, Max-Planck-Institut für Gravitationsphysik, D-14476 Golm, Germany

${ }^{20}$ Montana State University, Bozeman, Montana 59717, USA

${ }^{21}$ European Gravitational Observatory (EGO), I-56021 Cascina (PI), Italy

${ }^{22}$ Syracuse University, Syracuse, New York 13244, USA

${ }^{23}$ LIGO-Massachusetts Institute of Technology, Cambridge, Massachusetts 02139, USA

${ }^{24}$ APC, AstroParticule et Cosmologie, Université Paris Diderot, CNRS/IN2P3, CEA/Irfu, Observatoire de Paris,

Sorbonne Paris Cité, 10, rue Alice Domon et Léonie Duquet, 75205 Paris Cedex 13, France

${ }^{25}$ Columbia University, New York, New York 10027, USA

${ }^{26}$ Stanford University, Stanford, California 94305, USA

${ }^{27}$ IM-PAN, 00-956 Warsaw, Poland

${ }^{27 \mathrm{~b}}$ Astronomical Observatory Warsaw University, 00-478 Warsaw, Poland

${ }^{27 \mathrm{c}}$ CAMK-PAN, 00-716 Warsaw, Poland

${ }^{27 \mathrm{~d}}$ Biatystok University, 15-424 Biatystok, Poland

${ }^{27 \mathrm{e}}$ NCBJ, 05-400 Świerk-Otwock, Poland

${ }^{27 \mathrm{f}}$ Institute of Astronomy, 65-265 Zielona Góra, Poland

${ }^{28}$ The University of Texas at Brownsville, Brownsville, Texas 78520, USA

${ }^{29}$ San Jose State University, San Jose, California 95192, USA

${ }^{30}$ Moscow State University, Moscow 119992, Russia

${ }^{31 \mathrm{a}}$ LAL, Université Paris-Sud, IN2P3/CNRS, F-91898 Orsay, France

${ }^{31 \mathrm{~b}}$ ESPCI, CNRS, F-75005 Paris, France

${ }^{32}$ NASA/Goddard Space Flight Center, Greenbelt, Maryland 20771, USA

${ }^{33}$ University of Western Australia, Crawley, Western Australia 6009, Australia

${ }^{34}$ The Pennsylvania State University, University Park, Pennsylvania 16802, USA

${ }^{35 a}$ Université Nice-Sophia-Antipolis, CNRS, Observatoire de la Côte d'Azur, F-06304 Nice, France

${ }^{35 \mathrm{~b}}$ Institut de Physique de Rennes, CNRS, Université de Rennes 1, 35042 Rennes, France

${ }^{36}$ Laboratoire des Matériaux Avancés (LMA), IN2P3/CNRS, F-69622 Villeurbanne, Lyon, France

${ }^{37}$ Washington State University, Pullman, Washington 99164, USA

${ }^{38}$ INFN, Sezione di Perugia, I-06100 Perugia, Italy

${ }^{38 \mathrm{~b}}$ Università di Perugia, I-06123 Perugia, Italy 
${ }^{39 a}$ INFN, Sezione di Firenze, I-50019 Sesto Fiorentino, Italy

${ }^{39 \mathrm{~b}}$ Università degli Studi di Urbino 'Carlo Bo', I-61029 Urbino, Italy

${ }^{40}$ University of Oregon, Eugene, Oregon 97403, USA

${ }^{41}$ Laboratoire Kastler Brossel, ENS, CNRS, UPMC, Université Pierre et Marie Curie, 4 Place Jussieu, F-75005 Paris, France

${ }^{42}$ University of Maryland, College Park, Maryland 20742 USA

${ }^{43}$ Universitat de les Illes Balears, E-07122 Palma de Mallorca, Spain

${ }^{44}$ University of Massachusetts-Amherst, Amherst, Massachusetts 01003, USA

${ }^{45}$ Canadian Institute for Theoretical Astrophysics, University of Toronto, Toronto, Ontario M5S 3H8, Canada

${ }^{46}$ Tsinghua University, Beijing 100084, China

${ }^{47}$ University of Michigan, Ann Arbor, Michigan 48109, USA

${ }^{48}$ Louisiana State University, Baton Rouge, Louisiana 70803, USA

${ }^{49}$ The University of Mississippi, University, Mississippi 38677, USA

${ }^{50}$ Charles Sturt University, Wagga Wagga, New South Wales 2678, Australia

${ }^{51}$ Caltech-CaRT, Pasadena, California 91125, USA

${ }^{52}$ INFN, Sezione di Genova; I-16146 Genova, Italy

${ }^{53}$ Pusan National University, Busan 609-735, Korea

${ }^{54}$ Australian National University, Canberra, Australian Capital Territory 0200, Australia

${ }^{55}$ Carleton College, Northfield, Minnesota 55057, USA

${ }^{56}$ The University of Melbourne, Parkville, Victoria 3010, Australia

${ }^{57 a}$ INFN, Sezione di Roma Tor Vergata, I-00133 Roma, Italy

${ }^{57 b}$ Università di Roma Tor Vergata, I-00133 Roma, Italy

${ }^{57 c}$ Università dell'Aquila, I-67100 L'Aquila, Italy

${ }^{58}$ University of Salerno, I-84084 Fisciano (Salerno), Italy and INFN (Sezione di Napoli), I-80126 Napoli, Italy

${ }^{59}$ Instituto Nacional de Pesquisas Espaciais, 12227-010-São José dos Campos, Sao Paulo, Brazil

${ }^{60}$ The University of Sheffield, Sheffield S10 2TN, United Kingdom

${ }^{61}$ WIGNER RCP, RMKI, Konkoly Thege Miklós út 29-33, H-1121 Budapest, Hungary

${ }^{62}$ Inter-University Centre for Astronomy and Astrophysics, Pune 411007, India

${ }^{63}$ University of Minnesota, Minneapolis, Minnesota 55455, USA

${ }^{64 \mathrm{a}}$ INFN, Gruppo Collegato di Trento, I-38123 Povo, Trento, Italy

${ }^{64 \mathrm{~b}}$ Università di Trento, I-38050 Povo, Trento, Italy

${ }^{64 \mathrm{c}}$ INFN, Sezione di Padova, I-35131 Padova, Italy

${ }^{64 d}$ Università di Padova, I-35131 Padova, Italy

${ }^{65}$ California Institute of Technology, Pasadena, California 91125, USA

${ }^{66}$ Northwestern University, Evanston, Illinois 60208, USA

${ }^{67}$ Rochester Institute of Technology, Rochester, New York 14623, USA

${ }^{68}$ Eötvös Loránd University, Budapest 1117, Hungary

${ }^{69}$ University of Cambridge, Cambridge CB2 1TN, United Kingdom

${ }^{70}$ University of Szeged, 6720 Szeged, Dóm tér 9, Hungary

${ }^{71}$ Rutherford Appleton Laboratory, HSIC, Chilton, Didcot, Oxon OX11 OQX, United Kingdom

${ }^{72}$ Embry-Riddle Aeronautical University, Prescott, Arizona 86301, USA

${ }^{73}$ Perimeter Institute for Theoretical Physics, Ontario N2L 2Y5, Canada

${ }^{74}$ American University, Washington, DC 20016, USA

${ }^{75}$ University of New Hampshire, Durham, New Hampshire 03824, USA

${ }^{76}$ University of Southampton, Southampton SO17 1BJ, United Kingdom

${ }^{77}$ Korea Institute of Science and Technology Information, Daejeon 305-806, Korea

${ }^{78}$ Hobart and William Smith Colleges, Geneva, New York 14456, USA

${ }^{79}$ Institute of Applied Physics, Nizhny Novgorod 603950, Russia

${ }^{80}$ Lund Observatory, Box 43, SE-221 00 Lund, Sweden

${ }^{81}$ Hanyang University, Seoul 133-791, Korea

${ }^{82}$ Seoul National University, Seoul 151-742, Korea

${ }^{83}$ University of Strathclyde, Glasgow G1 $1 X Q$, United Kingdom

${ }^{84}$ The University of Texas at Austin, Austin, Texas 78712, USA

${ }^{85}$ Southern University and A\&M College, Baton Rouge, Louisiana 70813, USA

${ }^{86}$ University of Rochester, Rochester, New York 14627, USA

${ }^{87}$ University of Adelaide, Adelaide, South Australia 5005, Australia

${ }^{88}$ National Institute for Mathematical Sciences, Daejeon 305-390, Korea

${ }^{89}$ Louisiana Tech University, Ruston, Louisiana 71272, USA

${ }^{90}$ McNeese State University, Lake Charles, Louisiana 70609, USA

${ }^{91}$ Andrews University, Berrien Springs, Michigan 49104, USA

${ }^{92}$ Trinity University, San Antonio, Texas 78212, USA 


\footnotetext{
${ }^{93}$ University of Washington, Seattle, Washington 98195-4290, USA

${ }^{94}$ Southeastern Louisiana University, Hammond, Louisiana 70402, USA

${ }^{95}$ University of California at Berkeley, Berkeley, California 94720, USA
}

(Received 17 August 2012; published 13 February 2013)

This paper presents results of an all-sky search for periodic gravitational waves in the frequency range $[50,1190] \mathrm{Hz}$ and with frequency derivative range of $\sim[-20,1.1] \times 10^{-10} \mathrm{~Hz} \mathrm{~s}^{-1}$ for the fifth LIGO science run (S5). The search uses a noncoherent Hough-transform method to combine the information from coherent searches on time scales of about one day. Because these searches are very computationally intensive, they have been carried out with the Einstein@Home volunteer distributed computing project. Postprocessing identifies eight candidate signals; deeper follow-up studies rule them out. Hence, since no gravitational wave signals have been found, we report upper limits on the intrinsic gravitational wave strain amplitude $h_{0}$. For example, in the $0.5 \mathrm{~Hz}$-wide band at $152.5 \mathrm{~Hz}$, we can exclude the presence of signals with $h_{0}$ greater than $7.6 \times 10^{-25}$ at a $90 \%$ confidence level. This search is about a factor 3 more sensitive than the previous Einstein@Home search of early S5 LIGO data.

DOI: 10.1103/PhysRevD.87.042001

\section{INTRODUCTION}

A promising class of sources for detectable gravitational wave signals is rapidly rotating neutron stars with nonaxisymmetric deformations [1-5]. Such objects are expected to emit long-lived continuous-wave (CW) signals. In the rest frame of the neutron star, these waves have a constant amplitude and are quasimonochromatic with a slowly decreasing intrinsic frequency. They are received at Earth-based detectors with a Doppler modulation due to the relative motion between the source and the detector. Consequently the observed phase evolution depends on the intrinsic signal frequency, the first frequency time derivative (also called spin down), and the source sky position; these parameters shall collectively be called the phase evolution parameters. While using higher-order frequency derivatives could potentially improve the astrophysical detection efficiency in a part of the parameter space (see Sec. III), we shall not consider them in this paper for computational reasons. Finally, the received signal has a time-dependent amplitude modulation due to the (time-dependent) relative geometry of the wave and the detector.

The previous two decades have seen the construction and operation of several kilometer-scale laser interferometric gravitational wave detectors [6-11]. The detectors and the data analysis tools have steadily improved over this period. These have made it possible to search for various gravitational wave signals with ever-improving sensitivity. In this paper we focus on data from the fifth science run (S5) of the LIGO (Laser Interferometer Gravitational Wave Observatory) detectors, collected between the GPS times of 815155213 s (Fri Nov 0416:00:00 UTC 2005) and

Published by the American Physical Society under the terms of the Creative Commons Attribution 3.0 License. Further distribution of this work must maintain attribution to the author(s) and the published article's title, journal citation, and DOI.
PACS numbers: 04.80.Nn, 95.55.Ym, 97.60.Gb, 07.05.Kf

875145614 s (Sun Sep 3000:00:00 UTC 2007). The LIGO network [6] consists of three detectors: one at Livingston, Louisiana, USA, with an arm length of $4 \mathrm{~km}$ (L) and two in the same vacuum envelope at Hanford, Washington, USA, with arm lengths of $4 \mathrm{~km}(\mathrm{H})$ and $2 \mathrm{~km}$, respectively. Only data from $\mathrm{H}$ and $\mathrm{L}$ detectors are used in this paper. The Virgo and GEO 600 detectors also collected data during the same time interval but are not used in this analysis, which is optimized for two detectors with similar sensitivities.

A coherent strategy for extracting faint $\mathrm{CW}$ signals buried in noisy data using standard maximum-likelihood techniques in the presence of "nuisance parameters" was derived in Ref. [12]. The resulting detection statistic is the so-called $\mathcal{F}$-statistic, which has since been generalized to the case of multiple detectors $[13,14]$. The $\mathcal{F}$-statistic has also been shown to arise as a special case in a more general Bayesian framework [15]. Using the $\mathcal{F}$-statistic means that we need to search explicitly only over the phase evolution parameters.

Coherent wide-parameter-space searches utilizing the $\mathcal{F}$-statistic have been carried out since the second LIGO science run [16,17]. The amplitude sensitivity of this type of search improves as the square root of the time baseline. However, the template bank spacing decreases dramatically with the time baseline, and the computational requirements of the search increase rapidly. Even with a coherent time baseline of just a few days, a widefrequency-band all-sky search is computationally extremely challenging. It becomes completely unfeasible if one considers instead time baselines on the order of months.

As is often the case with computationally bound problems, hierarchical approaches have been proposed [18-20]. In these strategies, the entire data set is split into shorter segments. Each segment is analyzed coherently, and afterwards the information from the different segments is combined incoherently (which means that the phase 
information is lost). The amplitude sensitivity grows at best with the fourth root of the number of segments. Such methods have been used in previous wide-parameter-space searches published by the LIGO and Virgo Collaborations [21-26].

A subset of these searches [21,22,25,26] used segments sufficiently short (1800 s) that the signal remains within a single Fourier frequency bin in each segment. In these cases, a simple Fourier transform suffices for each segment. Three different variants of such methods have been developed that combine the results from the different short segments incoherently: the "stack-slide," the "Houghtransform" and the "PowerFlux" schemes. The stack-slide procedure $[18,20]$ averages the normalized power from the Fourier transform of 30-minute segments [short time baseline Fourier transform (SFT)] of the calibrated detector strain data. The PowerFlux scheme [22,25] can be seen as a variation of the stack-slide method, where the power is weighted before summing. The weights are chosen according to the detector noise and antenna pattern to maximize the signal-to-noise ratio (SNR). The Hough-transform method $[19,27]$ sums weighted binary counts, depending upon whether the normalized power in an SFT bin exceeds a certain threshold.

As the segment duration is increased, it becomes necessary to account for signal modulations within each segment by computing the $\mathcal{F}$-statistic over a grid in the space of phase evolution parameters. This results in a significant increase in the computational requirements of the search. The distributed volunteer computing project Einstein@Home [28] has been created to address this need. Two previous papers $[23,24]$ report on results of such CW searches from the fourth LIGO science run and from the first two months of S5, respectively. The method used was based on the computation of the coherent $\mathcal{F}$-statistic on data segments from either the $\mathrm{H}$ or $\mathrm{L}$ detectors separately, and only parameter space points with values of $2 \mathcal{F}$ larger than 25 were returned back to the Einstein@Home server for further inspection. The threshold value of 25 limited the ultimate sensitivity of that search: if a signal was not loud enough to surpass that threshold on at least some of the segments it would not be detected. The threshold value was set by bandwidth constraints on the size of the results file uploaded back to the server by the host, i.e., on the maximum number of significant points that could be returned. These results were subsequently combined by a coincidence scheme, performed offline in the postprocessing phase. In contrast, in the search presented here, the combination of the results from the coherent searches takes place directly on the host machines using a Hough-transform scheme. This makes it possible to use a much lower threshold on $2 \mathcal{F}$, equal to 5.2, that defines the parameter space points to be passed on to the Hough transform. Moreover, in this search, data from the $\mathrm{H}$ and $\mathrm{L}$ detectors are coherently combined $[13,14]$. Finally, more data was searched in this analysis compared to any previous Einstein@Home search. The Einstein@Home runs presented here refer to searches based on the first (S5R3) and second year of S5 LIGO data. This latter search was run on Einstein@Home in two separate steps, called S5R5 and S5R6. Since the S5R6 run used the same data as S5R5, but extended the search region above $1 \mathrm{kHz}$, in this paper we simply refer to these two runs as S5R5.

The paper is structured as follows. In Sec. II, we briefly review the Hough-transform method. Section III describes the Einstein@Home distributed search used to analyze the data set. Section IV gives a detailed description of the S5R5 postprocessing, which is based on the pioneer S5R3 postprocessing (described in Appendix B). Upper limit computations from the more-sensitive S5R5 data are provided in Sec. V. The study of some hardware-injected signals is presented in Sec. VI. In Sec. VII we make some concluding remarks.

\section{THE DATA ANALYSIS METHOD}

\section{A. The waveform model}

Let us begin by briefly describing the standard signal model for CW signals. In the rest frame of the neutron star, the gravitational wave signal is elliptically polarized with constant amplitudes $A_{+, \times}$for the two polarizations $h_{+, \times}(t)$. Thus, we can find a frame such that

$$
h_{+}(t)=A_{+} \cos \phi(t), \quad h_{\times}(t)=A_{\times} \sin \phi(t) .
$$

The two amplitudes are related to an overall amplitude $h_{0}$ and the inclination angle $\iota$ between the line of sight to the neutron star and its rotation axis,

$$
A_{+}=\frac{1}{2} h_{0}\left(1+\cos ^{2} \iota\right), \quad A_{\times}=h_{0} \cos \iota .
$$

The value of $h_{0}$ is model dependent. For emission due to nonaxisymmetric distortions, the amplitude $h_{0}$ depends on the ellipticity $\varepsilon$ of the star defined as

$$
\varepsilon=\frac{\left|I_{x x}-I_{y y}\right|}{I_{z z}}
$$

Here $I_{z z}$ is the principal moment of inertia of the star, and $I_{x x}$ and $I_{y y}$ are the moments of inertia about the other axes. The amplitude is given by

$$
h_{0}=\frac{4 \pi^{2} G}{c^{4}} \frac{I_{z z} f^{2} \varepsilon}{d},
$$

where $f$ is the frequency of the emitted GW signal (which is also twice the rotational frequency of the star), $G$ is Newton's constant, $c$ is the speed of light and $d$ is the distance to the star. The distribution of $\varepsilon$ for neutron stars is uncertain and model dependent since the breaking strain for a neutron star crust is highly uncertain (see, e.g., Refs. [2,29-31] for further discussion). 
Energy loss from the emission of gravitational and/or electromagnetic waves, as well as possible local acceleration of the source, causes the signal frequency arriving at the solar system to evolve. To first order, it can be expressed as

$$
\hat{f}(\tau)=f_{0}+\dot{f}\left(\tau-\tau_{0}\right),
$$

where $\tau$ is the arrival time of a wave front at the solar system barycenter ( $\mathrm{SSB}), f_{0}$ is the frequency at a fiducial reference time $\tau_{0}$, and $\dot{f}$ denotes the first time derivative of the frequency. The astrophysical implications of ignoring higher-order derivatives in this Taylor expansion will be discussed later. The phase of the signal, $\phi(t)$, follows directly from the frequency evolution with an initial phase $\phi_{0}$ at the reference time (see, e.g., Ref. [19]).

As the detector on the Earth moves relative to the SSB, the arrival time of a wave front at the detector, $t$, differs from the SSB time $\tau^{1}$ :

$$
\tau(t)=t+\frac{\vec{r}(t) \cdot \vec{n}}{c}+\Delta_{E \odot}-\Delta_{S \odot} .
$$

Here $\vec{r}(t)$ is the position vector of the detector in the SSB frame, and $\vec{n}$ is the unit vector pointing to the neutron star; $\Delta_{E \odot}$ and $\Delta_{S \odot}$ are respectively the relativistic Einstein and Shapiro time delays [32]. In standard equatorial coordinates with right ascension $\alpha$ and declination $\delta$, the components of the unit vector $\vec{n}$ are given by $(\cos \alpha \cos \delta, \sin \alpha \cos \delta, \sin \delta)$.

Ignoring the relativistic corrections, the instantaneous frequency $f(t)$ of a CW signal, as observed at time $t$ by a detector on Earth, is described by the well-known Doppler shift equation:

$$
f(t)=\hat{f}(\tau)+\hat{f}(\tau) \frac{\vec{v}(\tau) \cdot \vec{n}}{c},
$$

where $\vec{v}(\tau)$ is the detector velocity with respect to the SSB frame; $\vec{v}(\tau)$ is the sum of two components, from the yearly Earth motion around the Sun $\left(\vec{v}_{y}\right)$ and from the rotation of Earth around its axis $\left(\vec{v}_{d}\right)$.

Finally, the received signal at the detector is

$$
h(t)=F_{+}(t ; \vec{n}, \psi) h_{+}(t)+F_{\times}(t ; \vec{n}, \psi) h_{\times}(t),
$$

where $F_{+, \times}$are the detector beam pattern functions which depend on the sky position $\vec{n}$ and the relative polarization angle $\psi$ of the wave frame $[12,33]$. The wave frame is a right-handed Cartesian coordinate system based on the direction of propagation of the gravitational wave. Its $z$ axis is along the direction of propagation, and its $x$ and $y$ axes are along the principal directions of polarization of the wave. There are thus altogether eight signal parameters, which include the four phase evolution parameters $\left(f_{0}, \dot{f}, \alpha, \delta\right)$, and four other parameters $\left(h_{0}, \iota, \psi, \phi_{0}\right)$.

\footnotetext{
${ }^{1}$ Proper motion of the source can safely be neglected for distances greater than $\sim 10 \mathrm{pc}$.
}

\section{B. The Hough-transform algorithm}

For completeness, we summarize the Hough detection statistic in this section. Further details of the method are given in Ref. [19] and previous searches with this method applied to short coherent times are reported in Refs. [21,22].

The Hough transform is a well-known technique used mainly in digital image processing for robust extraction of patterns. Such a procedure is employed here for identifying points in the time-frequency plane that match the pattern expected from a signal. The time-frequency data in our case is the $\mathcal{F}$-statistic computed as a function of signal frequency, for each of the data segments of duration $T_{\text {seg }}$, over a grid of points in the space of $(\alpha, \delta, \dot{f})$. The grid in $(\alpha, \delta, \dot{f})$ space used for this $\mathcal{F}$-statistic computation is called the coarse grid because its resolution is determined by the coherent time baseline $T_{\text {seg }}$ and makes no reference to the full observation time or the number of segments $N_{\mathrm{seg}}$. The result of this computation is thus a collection of $\mathcal{F}$-statistic values $\mathcal{F}_{\alpha, \delta, f}^{i}(k)$ where the integers $k$ and $i$ label a frequency bin (with spacing $\delta f$ as defined below) and a data segment, respectively.

The frequency and frequency derivative spacings for the coarse grid are based on choosing the maximum allowed fractional loss in the $\mathcal{F}$-statistic when the signal and template points are slightly mismatched. This leads naturally to the notion of a metric in parameter space $[34,35]$ and this has been studied for the CW case in Ref. [14]. The grid spacings in $f, \dot{f}$ are given respectively by $[36,37]$

$$
\delta f=\frac{\sqrt{12 m}}{\pi T_{\mathrm{seg}}}
$$

and

$$
\delta \dot{f}=\frac{\sqrt{720 m}}{\pi T_{\mathrm{seg}}^{2}},
$$

where $m$ represents the nominal single dimension mismatch value [14,38]. For all the Einstein@Home runs discussed here, $m$ has been taken equal to 0.3. The span $T_{\text {seg }}$ of each segment has been set equal to 25 hours for all the runs. The frequency resolution, given by Eq. (9), is $\delta f \sim 6.7 \mu \mathrm{Hz}$ for all the Einstein@ Home runs described in this paper. As we shall see shortly, for technical reasons it turned out to be necessary to use a finer spacing for $\dot{f}$ than given by Eq. (10).

In combining these $N_{\text {seg }}$ different $\mathcal{F}$-statistic vectors, it is necessary to use a finer grid in $(\alpha, \delta, \dot{f})$ centered around each coarse grid point. Our implementation of the Houghtransform algorithm assumes that the fine grid is a Cartesian product of a rectangular sky grid and one dimensional grids in $f$ and $\dot{f}$. Moreover the fine sky grid is assumed to be aligned with the $\alpha$ and $\delta$ directions in the sky. In order to completely cover the sky with the different 


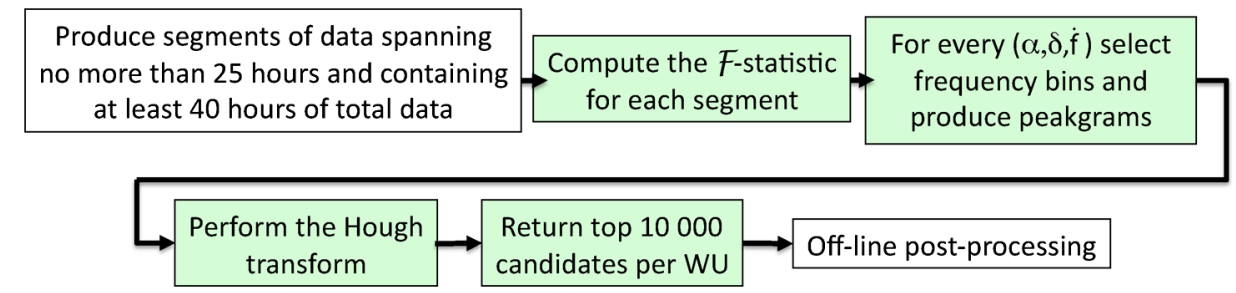

FIG. 1 (color online). High-level schematic of the pipeline used in the searches. For each data segment, the multi-detector $\mathcal{F}$-statistic is computed and frequency bins are selected setting a threshold on the $\mathcal{F}$-statistic. Such selected frequency bins are then used to create the Hough map. The output is a set of candidates in the parameter space. The color-filled boxes indicate the steps performed on the volunteer computers. The acronym "WU" is defined in Sec. III A and refers to the independent computing tasks into which we partition the computational work of the search.

fine sky grids, it is thus simplest to choose a rectangular coarse sky grid aligned with the $(\alpha, \delta)$ directions. We choose a coarse grid such that the spacing in $\delta$ is a constant and the spacing in $\alpha$ is proportional to $(\cos \delta)^{-1}$. This ensures that each cell of the coarse grid covers a fixed solid angle.

Since the coherent integration time is very close to a sidereal day, it is reasonable to assume that the Hough skypatch size $d \theta$ (which in our case is the same as the coarse sky-grid resolution) is determined by $v_{d}$, the Earth's rotation speed at the equator. At frequency $\hat{f}$ we have [19]

$$
d \theta=\frac{c}{v_{d} \hat{f} T_{\mathrm{seg}}} .
$$

In practice, this estimate was verified by Monte-Carlo studies with signal injections, and we used

$$
d \theta_{\mathcal{F}}= \begin{cases}d \theta & \text { for S5R3 } \\ \mathcal{R} d \theta & \text { for S5R5 }\end{cases}
$$

where the factor $\mathcal{R}=\sqrt{3}$ increases the size of the sky patch $d \theta$ for the S5R5 run. The Monte-Carlo studies showed that this grid spacing for S5R5 corresponded approximately to a mismatch of $m \approx 0.3$ so that, for any other value of $m$, the spacing should be approximately $\sqrt{m / 0.3} \mathcal{R} d \theta$.

We also need to set the resolution of the refined sky grid used by the Hough algorithm. Since the full observation time is of the order of a year, the relevant scale here is set by the speed $v_{y}$ of Earth as it orbits the Sun. Following [19], the resolution for the fine sky grid at a frequency $\hat{f}$ is given by

$$
d \theta_{\mathrm{H}}=\frac{c \delta f}{\wp \hat{f} v_{y}}
$$

The parameter $\wp$ scales the resolution compared to the conservative estimate $c \delta f /\left(\hat{f} v_{y}\right)$ and in practice, again based on Monte-Carlo studies, we used $\wp=0.5$. One can see that the increase in the number of sky position points from the coarse $\mathcal{F}$-statistic grid to the fine Hough grid is
$\mathcal{N}_{\text {sky }}^{\text {ref }}=\left(d \theta_{\mathcal{F}} / d \theta_{\mathrm{H}}\right)^{2}=\left(\frac{\pi}{\sqrt{12 m}} \wp \frac{v_{y}}{v_{d}}\right)^{2} \sim \mathcal{O}\left(10^{4}\right)$

Taking $m=0.3$ and $\wp=0.5$ yields $\mathcal{N}_{\text {sky }}^{\text {ref }} \simeq 8444$ for the S5R5 run.

Finally, let us turn to the coarse and fine grids for $\dot{f}$. Ideally, we should refine the coarse $\dot{f}$ grid spacing of Eq. (10) by a factor $N_{\text {seg }}$ [19]. However, in our implementation of the Hough transform, using this refinement turns out to increase the maximum memory footprint of the different searches and would make it unsuitable for Einstein@Home. As a compromise, it was decided not to use any refinement in $\dot{f}$ and instead to use a finer resolution for the coarse grid. Based on Monte-Carlo analyses, an acceptable value for the $\dot{f}$ spacing turns out to be

$$
(\delta \dot{f})_{\mathrm{S} 5 \mathrm{R} 5}=\frac{\sqrt{3.3 m}}{T_{\mathrm{seg}}^{2}} \sim 1.2 \times 10^{-10} \mathrm{~Hz} \mathrm{~s}^{-1} .
$$

This value was used for S5R5. However, for S5R3, this was incorrectly set to

$$
(\delta \dot{f})_{\mathrm{S} 5 \mathrm{R} 3}=\frac{\sqrt{33 m}}{T_{\mathrm{seg}}^{2}} \sim 3.8 \times 10^{-10} \mathrm{~Hz} \mathrm{~s}^{-1},
$$

leading to a corresponding loss in sensitivity for S5R3.

The flow chart of the search algorithm used for this search is depicted in Fig. 1. The input data set is composed of 30-minute baseline SFTs. This set is partitioned in subsets such that no more than 25 hours of data are spanned by each segment and such that there is overall (including data from both detectors) at least 40 hours of data in each segment. Let $T_{\mathrm{obs}}$ be the observation time spanned by the $N_{\text {seg }}$ segments constructed in this way. ${ }^{2}$ The multidetector $\mathcal{F}$-statistic is computed for each segment at each point of the search parameter space $(f, \dot{f}, \alpha, \delta)$. The next step consists of selecting parameter space points for which the $\mathcal{F}$-statistic is above the fixed threshold. For every set of

\footnotetext{
${ }^{2}$ Note that $T_{\text {seg }} \neq T_{\text {obs }} / N_{\text {seg }}$ because of the gaps that are unavoidably present in the data stream, corresponding to times when the interferometers were not in lock, and to the selection of 25-hour segments containing the requisite amount of data.
} 
$(f, \dot{f}, \alpha, \delta)$, we assign a value $n_{i}=1$ or 0 in the $i$ th segment depending on whether the corresponding $\mathcal{F}$-statistic is above the threshold 5.2 or not; this threshold turns out to be optimal [19]. The values $n_{i}(f)$ are called a "peakgram," which is the input to the Hough transform.

The final statistic used by the Hough search is a weighted sum of binary counts $n_{i}$, giving the so-called Hough number count $n_{\mathrm{c}}$, expressed by [22]

$$
n_{\mathrm{c}}=\sum_{i=0}^{N_{\mathrm{seg}}-1} w_{i} n_{i}
$$

The weight $w_{i}$ for a frequency $f$ and for a particular sky location is determined from the average antenna response and average detector noise over the duration of the $i$ th segment. Since the input data in each segment consists of SFTs, we perform the averaging over each SFT. Let $N_{i}$ be the number of SFTs in the $i$ th segment. Let $S_{h}^{i, \gamma}$ be the single-sided power spectral density (PSD) of the $\gamma$ th SFT in the $i$ th segment, averaged over a narrow frequency band containing the search frequency. The $w_{i}$ are given by

$$
w_{i} \propto \sum_{\gamma=0}^{N_{i}-1}\left(F_{+(i, \gamma)}^{2}+F_{\times(i, \gamma)}^{2}\right) w_{i, \gamma},
$$

where $F_{+(i, \gamma)}$ and $F_{\times(i, \gamma)}$ are the detector antenna pattern functions for the $\gamma$ th SFT in the $i$ th segment, and

$$
w_{i, \gamma}=\frac{1}{S_{h}^{i, \gamma}} \times\left(\frac{1}{N_{i}} \sum_{\beta=0}^{N_{i}-1} \frac{1}{S_{h}^{i, \beta}}\right)^{-1} .
$$

It is easy to see that in the hypothetical case when the data is exactly stationary, so all the $S_{h}^{i, \gamma}$ are identically equal to each other, then $w_{i, \gamma}=1$. More realistically, the $w_{i, \gamma}$ are approximately unity for stationary data and the use of the harmonic mean in Eq. (19) ensures that the $w_{i, \gamma}$ do not deviate too far from unity in the presence of nonstationary noise.

The weight normalization is

$$
\sum_{i=0}^{N_{\mathrm{seg}}-1} w_{i}=N_{\mathrm{seg}}
$$

which ensures that the Hough number count $n_{\mathrm{c}}$ lies within the range $\left[0, N_{\mathrm{seg}}\right]$. In Ref. [22] it is shown that the weights $w_{i}$, first derived in Ref. [39], maximize the sensitivity, averaged over the orientation of the source (see Appendix A for a further discussion of the weights and some technical problems that were encountered in the search).

From the Hough number count $n_{\mathrm{c}}$ we define the significance (or critical ratio), $\mathrm{CR}$,

$$
\mathrm{CR}=\frac{n_{\mathrm{c}}-\bar{n}_{\mathrm{c}}}{\sigma},
$$

which measures the significance of $n_{\mathrm{c}}$ as the deviation from the expected value $\bar{n}_{\mathrm{c}}$, measured in units of the noise fluctuations $\sigma$ in Gaussian stationary noise in the absence of any signal. In these circumstances, $\bar{n}_{\mathrm{c}}=N_{\mathrm{seg}} p$ and $p$ is the probability that a $\chi^{2}$ variable with four degrees of freedom (as $2 \mathcal{F}$ in absence of a signal) exceeds the threshold of 5.2. In case of unity weighting, the standard deviation is simply that of the binomial distribution: $\sigma=\sqrt{N_{\text {seg }} p(1-p)}$. When the weights are used, the standard deviation is given by

$$
\sigma=\sqrt{\|\vec{w}\|^{2} p(1-p)}
$$

where $\|\vec{w}\|^{2}=\sum_{i=0}^{N_{\text {seg }}-1} w_{i}^{2}$ [22]. The CR is the detection statistic returned by the hierarchical searches presented here.

\section{THE EINSTEIN@HOME DISTRIBUTED SEARCH}

The Einstein@Home project is built upon the BOINC (Berkeley Open Infrastructure for Network Computing) architecture [40-42], a system that exploits the idle time on volunteer computers to solve scientific problems that require large amounts of computer power. During the S5R5 run, Einstein@Home had approximately 225000 registered volunteers and approximately 750000 registered host machines that contributed a total of approximately 25000 CPU (central processing unit) years.

\section{A. Details of the S5R5 search}

The computational load is partitioned in independent computing tasks, called "workunits" (WUs), each of which is analyzed by a volunteer machine. In particular, 7369434 and 10945955 WUs have been generated for the S5R3 and S5R5 runs, respectively. The S5R5 run was launched on January 13, 2009 and ended on October 30, 2009. It used 10560 S5 LIGO SFTs, collected between the GPS times of 852443819 s (Wed Jan 1005:56:45 GMT 2007) and 875278812 s (Mon Oct 0112:59:58 GMT 2007). The analyzed data consists of 5550 and 5010 SFTs from the LIGO $\mathrm{H}$ and $\mathrm{L}$ interferometers, respectively. The number of data segments used for the S5R5 run is 121, each spanning no more than $T_{\text {seg }}=25$ hours and with at least 40 hours of data, as already said. Similar details for the S5R3 run can be found in Appendix B.

The total search frequency range of the S5R5 search is $[50,1190] \mathrm{Hz}$, with a frequency resolution $\delta f \sim 6.7 \mu \mathrm{Hz}$ and spin-down resolution $\delta \dot{f} \sim 0.12 \mathrm{nHz} \mathrm{s}^{-1}$. Each WU analyzes a constant frequency band $B \simeq 20 \mathrm{mHz}$, the full spin- down interval, ranging roughly from $-2 \mathrm{nHz} \mathrm{s}^{-1}$ to $0.11 \mathrm{nHz} \mathrm{s}^{-1}$, and a region of the sky, as we shall see in Sec. III C.

The original data contained instrumental artifacts in narrow frequency bands that were known before the launch of the Einstein@Home run. Those bands were identified 
TABLE I. Instrumental lines identified and "cleaned" before the Einstein@Home runs. The different columns represent (I) the source of the line; (II) the central frequency of the instrumental line; (III) the number of harmonics; (IV) low-frequency-side (LFS) of the knockout band; (V) high-frequency-side (HFS) of the knockout band; (VI) the interferometer where the instrumental lines were identified. Note that when there are higher harmonics, the knockout band width remains constant.

\begin{tabular}{llcccc}
\hline \hline Cause & $f_{\mathrm{L}}(\mathrm{Hz})$ & Harmonics & LFS $(\mathrm{Hz})$ & HFS $(\mathrm{Hz})$ & $\mathrm{IFO}$ \\
\hline Calibration & 46.7 & 1 & 0.0 & 0.0 & $\mathrm{H}$ \\
Calibration & 54.7 & 1 & 0.0 & 0.0 & $\mathrm{~L}$ \\
Mains & 60 & 19 & 1 & 1 & $\mathrm{H}, \mathrm{L}$ \\
Wire & 345 & 1 & 5 & 5 & $\mathrm{~L}$ \\
Wire & 346 & 1 & 4 & 4 & $\mathrm{H}$ \\
Calibration & 393.1 & 1 & 0.0 & 0.0 & $\mathrm{H}$ \\
Calibration & 396.7 & 1 & 0.0 & 0.0 & $\mathrm{~L}$ \\
Wire & 686.5 & 1 & 1.0 & 1.0 & $\mathrm{~L}$ \\
Wire & 686.9 & 1 & 0.3 & 0.3 & $\mathrm{H}$ \\
Wire & 688.2 & 1 & 0.3 & 0.3 & $\mathrm{H}$ \\
Wire & 689.5 & 1 & 0.5 & 0.6 & $\mathrm{H}$ \\
Wire & 693.7 & 1 & 0.7 & 0.7 & $\mathrm{~L}$ \\
Wire & 694.75 & 1 & 1.25 & 1.25 & $\mathrm{H}$ \\
Wire & 1029.5 & 1 & 0.25 & 0.25 & $\mathrm{~L}$ \\
Wire & 1030.55 & 1 & 0.1 & 0.1 & $\mathrm{H}$ \\
Wire & 1031.0 & 1 & 0.5 & 0.5 & $\mathrm{~L}$ \\
Wire & 1032.18 & 1 & 0.04 & 0.04 & $\mathrm{H}$ \\
Wire & 1032.58 & 1 & 0.1 & 0.1 & $\mathrm{H}$ \\
Wire & 1033.6 & 1 & 0.2 & 0.2 & $\mathrm{~L}$ \\
Wire & 1033.7 & 1 & 0.1 & 0.1 & $\mathrm{H}$ \\
Wire & 1033.855 & 1 & 0.05 & 0.05 & $\mathrm{H}$ \\
Wire & 1034.6 & 1 & 0.4 & 0.4 & $\mathrm{H}$ \\
Wire & 1041.0 & 1 & 1.0 & 1.0 & $\mathrm{~L}$ \\
Wire & 1041.23 & 1 & 0.1 & 0.1 & $\mathrm{H}$ \\
Wire & 1042.00 & 1 & 0.5 & 0.2 & $\mathrm{H}$ \\
Wire & 1043.4 & 1 & 0.2 & 0.2 & $\mathrm{H}$ \\
Calibration & 1144.3 & 1 & 0.0 & 0.0 & $\mathrm{H}$ \\
Calibration & 1151.5 & 1 & 0.0 & 0.0 & $\mathrm{~L}$ \\
\hline \hline & & 1 & & & \\
\hline
\end{tabular}

and the corresponding frequency bins in the SFTs were replaced with white Gaussian noise at the same level as the neighboring frequencies. Table I shows which bands were treated in this manner and what instrumental artifact they harbored. These control bands are useful to compare and contrast the results obtained on real data against pure theoretical noise. Measurements and studies after the Einstein@Home run refined the frequencies and widths of these artifacts and identified additional ones; the final lists of artifacts are given in Appendix C, and were used to discard candidates (as we shall see in Sec. IV B). The "cleaning" process affected $\sim 27 \mathrm{~Hz}$ of search bandwidth, in addition to bands that were eliminated later in postprocessing.

The output data files from each WU are stored as ZIPcompressed ASCII text files containing the 10000 most significant candidates ranked according to the significance [as defined in Eq. (21)] over the parameter space searched by that WU. The decision to keep the top 10000 candidates was based on the maximum upload volume from the hosts to the Einstein@Home servers. All in all, on the order of $10^{11}$ candidates were returned to the Einstein@Home server from each run, corresponding roughly to $2.3 \mathrm{~TB}$ of data.

The files contain nine quantities for each candidate. The first four are the values (on the coarse grids) of the frequency $f$, sky position $(\alpha, \delta)$, and spin down $\dot{f}$. The fifth is the significance of the candidate as defined in Eq. (21). The remaining four quantities are connected with the refined sky grid centered on each coarse sky-grid point (recall that refinement is performed only on the sky). In particular, the location of the most significant point on the fine sky grid and the mean and standard deviation of the Hough number count values on all points of the fine grid are returned.

\section{B. Validation of returned candidates}

In order to eliminate potential errors, due to defective hardware and/or software or to fraud, BOINC is configured so that each WU is processed redundantly by computers owned by at least two different volunteers. An automated validation process checks the consistency of the results, ruling out those that are inconsistent, in which case new WUs are generated to run again independently. The first step of the validation is to check that the file syntax is correct and that the first four values, i.e., $(f, \alpha, \delta, \dot{f})$, are within the appropriate ranges. Next, for the pair of result files from each WU, the validator checks that the values of $(f, \alpha, \delta, \dot{f})$ agree to within floating point accuracy (the frequency is in fact checked to double precision). Finally, the significance values $\mathrm{CR}_{1}$ and $\mathrm{CR}_{2}$ from the two result files are compared and are validated if

$$
\Delta:=\left|\mathrm{CR}_{1}-\mathrm{CR}_{2}\right| /\left(\mathrm{CR}_{1}+\mathrm{CR}_{2}\right)<0.12 .
$$

In S5R5, about $0.045 \%$ of results that were processed by the validator were marked as "invalid," including both syntax errors in individual files and errors in comparisons of different results files. Excluding the syntax errors in individual files, the error rate arising from comparisons of pairs of distinct result files (most likely due to differences in floating point arithmetic on different computational platforms) was $\sim 0.015 \%$.

Is it possible that two invalid results could agree with each other and thus end up being marked as valid? While it is difficult to exclude this scenario with complete certainty, an upper limit for the probability of this happening is $(0.015 / 100)^{2} \approx 2.2 \times 10^{-8}$ (only the $0.015 \%$ error rate due to comparisons of distinct result files is relevant here). As mentioned earlier, there were a total of $\sim 1.1 \times$ $10^{7}$ WUs. It is therefore unlikely that even a single pair of result files would be incorrect and still pass validation.

The threshold of 0.12 on the value of $\Delta$ defined above turns out to be much looser than necessary. The differences 
in the actual observed values of $\Delta$ from a pair of matching result files are usually much smaller. The observed standard deviation of $\Delta$ turns out to be $\sim 0.012$, i.e., an order of magnitude smaller than the threshold. In addition, the standard deviation of the difference $\mathrm{CR}_{1}-\mathrm{CR}_{2}$ is measured to be $\sim 0.15$, which corresponds to a standard deviation of $\sim 0.7$ in terms of the number count. As we shall see later (see, e.g., Fig. 3), the loudest events in every $0.5 \mathrm{~Hz}$ band have an average loudest number count $\gtrsim 70$. Thus, these differences correspond to a $\lesssim 1 \%$ effect in the number count at the 1- $\sigma$ level, and we expect this to have a negligible effect on our analysis.

\section{Workunit design}

The design of the WUs must satisfy certain requirements. The first is that the WUs must be balanced, i.e., each WU must cover the same number of parameter space points so that they can be completed in roughly the same amount of time by a typical host machine. Second, the amount of data that must be downloaded by each host machine and the maximum memory footprint of each job must be within appropriate limits. Finally, one needs to choose the computational time for each WU on a typical host machine and the total time that the project should run, given the total computational power that is available. To meet these requirements, we need to understand how to split up the parameter space, and to measure the CPU core time spent by the search code on each part of the analysis.

We start with the basic parameters of the search, namely the total observation time $T_{\text {obs }}$, the coherent time baseline $T_{\text {seg }}$, the number of segments $N_{\text {seg }}$, the resolution for the coarse and fine sky grids, $d \theta_{\mathcal{F}}$ and $d \theta_{H}$, given by Eqs. (12) and (13), respectively, and the frequency and spin-down resolutions, $\delta f$ and $\delta \dot{f}$, given by Eqs. (9) and (15), respectively. Recall that the limit on the maximum memory footprint of each job already forced us to forego any refinement in $\dot{f}$.

Unlike the previous Einstein@Home search [24], where each WU searched the whole sky, here we choose each WU to cover a fixed frequency bandwidth $B$, the entire spindown search range, and a limited area of the sky. Let $\hat{f}_{b}$ be the highest frequency in the $b$ th search band. We want the computation time for every WU to be approximately the same, hence every WU must search the same number of coarse sky-grid points. Since the resolution in the sky, $d \theta_{\mathcal{F}}$, is inversely proportional to the frequency of the signal that we are searching for, WUs at higher frequencies will be searching smaller portions of the sky. Let $N_{b}$ be the number of coarse grid sky points over the whole sky for the $b$ th band,

$$
N_{b}=\frac{4 \pi}{d \theta_{\mathcal{F}}^{2}}=4 \pi\left(\frac{v_{d}}{c}\right)^{2} \frac{\hat{f}_{b}^{2} T_{\mathrm{seg}}^{2}}{\mathcal{R}^{2}}
$$

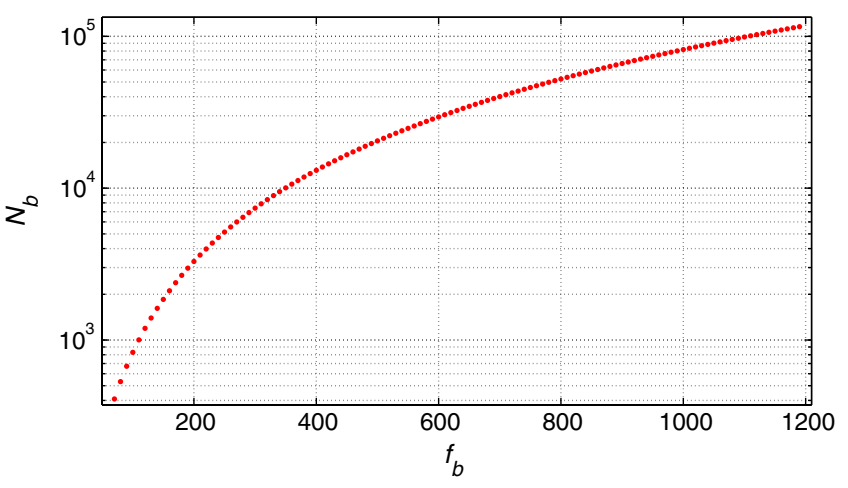

FIG. 2 (color online). Total number of $\mathcal{F}$-sky-grid points, for the S5R5 run, as a function of the frequency, given by Eq. (24).

which is shown in Fig. 2 for the S5R5 run. At frequency $\hat{f}_{b}$, we then partition the sky in $P_{b}$ parts, each containing $N_{\text {sky }}$ points,

$$
P_{b}=\frac{N_{b}}{N_{\mathrm{sky}}}=K \hat{f}_{b}^{2}
$$

with $K=4 \pi\left(\frac{v_{d}}{c}\right)^{2} T_{\text {seg }}^{2} \mathcal{R}^{-2} N_{\text {sky }}^{-1}$. In practice, to limit the number of sky-grid files needing to be downloaded by the host machines, the sky grids are constant over $10 \mathrm{~Hz}$ frequency bands and are determined based on the highest frequency in each band. A sky-grid file is a two-column ASCII file which specifies the positions in the sky (right ascension and declination) of the coarse grid templates to be searched for every frequency and spin-down value in a given $10 \mathrm{~Hz}$ band.

We choose a constant range of $\dot{f}$ values over the entire frequency band. To see what this implies for potential sources, we define the spin-down age for a system emitting at a frequency $f$ and spin down $\dot{f}$ to be $\tau=f /|\dot{f}|{ }^{3}$ It is clear that at a given search frequency, the minimum spindown age is determined by the maximum value of $|\dot{f}|$ included in the search. Our choice of a constant range of $\dot{f}$ means that the minimum spin-down age is frequency dependent,

$$
\tau_{\min }^{b}=\frac{f_{b}}{|\dot{f}|_{\max }} .
$$

We choose the minimum age at $50 \mathrm{~Hz}$ to be 800 years and a fixed range in $\dot{f}, \sim[-20,1.1] \times 10^{-10} \mathrm{~Hz} \mathrm{~s}^{-1}$.

\footnotetext{
${ }^{3}$ The reader is warned that the spin-down age as defined here can be rather different from the true chronological age of the star. For a neutron star with a present day frequency $f$ and spin down $\dot{f}$ due purely to the emission of GWs, the age would be $f / 4|\dot{f}|$; more generally this depends on the physical mechanisms that are responsible for the spin down [43]. Moreover, the notion of a spin-down age does not make sense for cases when $\dot{f}>0$. This might happen either due to accretion, or apparent spin up from acceleration in the vicinity of a globular cluster core, or because of proper motion leading to an apparently positive measured value of $\dot{f}$.
} 
The total number of WUs is simply the sum of the total number of sky partitions at each frequency $f_{b}$,

$$
\begin{aligned}
N_{\mathrm{WU}} & =\sum_{b} P_{b}=K \sum_{b} \hat{f}_{b}^{2} \approx \frac{K}{B} \int_{f_{\min }}^{f_{\max }} \hat{f}^{2} d f \\
& =\frac{K}{3 B}\left(f_{\max }^{3}-f_{\min }^{3}\right) .
\end{aligned}
$$

In the third step we have replaced the sum over frequencies with an integral between a minimum $\left(f_{\min }\right)$ and maximum $\left(f_{\max }\right)$ frequency. As a consistency check, we shall see later that $B \simeq 20 \mathrm{mHz}$, which is sufficiently small that this is a good approximation.

The total computing time for one WU can be expressed as

$$
\tau_{\mathrm{WU}}=N_{\mathrm{sky}} N_{\dot{f}}\left[\left(N_{f}+N_{\mathrm{sb}}\right) \tau_{\mathcal{F}}+N_{f} \tau_{\mathrm{H}}\right],
$$

where $N_{f}, N_{f}$, and $N_{\text {sky }}$ represent the number of coarse search frequency bins, spin-down values, and sky-grid points, respectively, $\tau_{\mathcal{F}}$ and $\tau_{\mathrm{H}}$ are the times needed to compute the $\mathcal{F}$-statistics and Hough number count for one point of the coarse grid; $N_{\mathrm{sb}}{ }^{4}$ represents additional "sideband" bins needed to compute the Hough-transform. The need for these sidebands can be understood by thinking about computing the Hough number count for a frequency near the edge of a given search band: that number count will involve summing the peakgrams along a curved track that can extend a small distance to either side of the target frequency.

The time $\tau_{\mathcal{F}}$ needed to compute the $\mathcal{F}$-statistic for one point of the coarse grid can be expressed as

$$
\tau_{\mathcal{F}}=\tau_{\mathcal{F}}^{1} N_{\mathrm{SFT}},
$$

where $N_{\mathrm{SFT}}$ is the total number of SFTs used in the search; $\tau_{\mathcal{F}}^{1}$ is the time needed to compute the $\mathcal{F}$-statistic per single SFT. The time $\tau_{\mathrm{H}}$ in Eq. (28), needed to compute the Hough number count corresponding to one point of the coarse grid, can be written as

$$
\tau_{\mathrm{H}}=\tau_{\mathrm{H}}^{1} N_{\text {seg }} \mathcal{N}_{\text {sky }}^{\text {ref }}
$$

where $\tau_{\mathrm{H}}^{1}$ is the time to sum the Hough number count per single data segment and per single point of the fine grid. Here $\mathcal{N}_{\text {sky }}^{\text {ref }}$ is an overall refinement factor, i.e., the number of grid points analyzed by the Hough algorithm for each

\footnotetext{
${ }^{4}$ The bins $N_{\mathrm{sb}}$ can be calculated by $\Delta f_{\mathrm{sb}} / \delta f$, where the average Hough "sidebands" $\left\langle\Delta f_{\mathrm{sb}}\right\rangle$ can be estimated from the Hough master equation. The frequency offset corresponding to the half diagonal distance $\Delta \vec{n}$, over one Hough sky patch, is $\left\langle\Delta f_{\mathrm{sb}}\right\rangle=(\hat{f} / c)\left\langle\left|\vec{v}_{y} \cdot \Delta \vec{n}\right|\right\rangle=\frac{v_{y}}{v_{d}} \frac{1}{\sqrt{2} T_{\text {seg }}}$. By using $T_{\text {seg }}=25$ hours, we get for S5R3 $\left\langle\Delta f_{\mathrm{sb}}\right\rangle \sim 5 \times 10^{-4} \mathrm{~Hz}$ and $N_{\mathrm{sb}} \sim 75$ bins, while for S5R5 $\left\langle\Delta f_{\mathrm{sb}}\right\rangle \sim 8.7 \times 10^{-4} \mathrm{~Hz}$ and $N_{\mathrm{sb}} \sim 130$ bins. However, to be conservative, $\left(2 \times N_{\mathrm{sb}}\right)$ bins were used for safety reasons and to take into account the changes of the velocity on the different segments.
}

coarse grid point. In our case, since the only refinement is over the sky, $\mathcal{N}_{\text {sky }}^{\text {ref }}$ is given by Eq. (14). The computational time is thus determined by the two timing constants $\tau_{\mathcal{F}}^{1}$ and $\tau_{\mathrm{H}}^{1}$. For our implementation of the algorithm, these constants were measured to be

$$
\tau_{\mathcal{F}}^{1}=180 \mathrm{~ns}, \quad \tau_{\mathrm{H}}^{1}=1.1 \mathrm{~ns} .
$$

The typical run time is found to vary by about $30 \%$ among different machines (at the 1-sigma level). The numbers in Eq. (31) are of course only average values for a typical host CPU core available at the time of the Einstein@Home runs.

The presence of $N_{\mathrm{sb}}$ leads to an overhead for the computation. We want to control this overhead and keep it below some acceptable level. Thus, we define the overhead $\epsilon$ to be the ratio between the time spent in computing the $\mathcal{F}$-statistic for the "sidebands" and the total computational time,

$$
\epsilon \equiv \frac{\Delta f_{\mathrm{sb}} \tau_{\mathcal{F}}}{B\left(\tau_{\mathcal{F}}+\tau_{\mathrm{H}}\right)+\Delta f_{\mathrm{sb}} \tau_{\mathcal{F}}} .
$$

The bandwidth $B$ needs to be sufficiently large so that $\epsilon$ is sufficiently small, but a too large value of $B$ can lead to excessively high download volumes for the Einstein@Home clients. From the above equation, we see that the frequency bandwidth $B$ can be determined by fixing $\epsilon$,

$$
B=\frac{\Delta f_{\mathrm{sb}} \tau_{\mathcal{F}}}{\tau_{\mathcal{F}}+\tau_{\mathrm{H}}}\left(\frac{1-\epsilon}{\epsilon}\right) .
$$

For all the Einstein@Home runs presented here we choose $\epsilon=5 \%$. For the S5R5 run, this leads to $B \simeq 20 \mathrm{mHz}$.

The total run time $\tau_{p}$ of the project is

$$
\tau_{p}=\frac{\tau_{\mathrm{WU}} N_{\mathrm{WU}}}{N_{\mathrm{CPU}}},
$$

where $N_{\mathrm{CPU}}$ represents the number of volunteer CPU cores. Given a certain number of CPU cores and having fixed $\tau_{p}$, the maximum search frequency $f_{\max }$ can be derived from the above equations to be

$$
f_{\max }^{3}=f_{\min }^{3}+3 \frac{\tau_{p} N_{\mathrm{CPU}}}{\tau_{\mathcal{F}}+\tau_{\mathrm{H}}} \frac{(1-\epsilon) \delta f}{\kappa N_{\dot{f}}},
$$

where $\kappa=4 \pi\left(\frac{v_{d}}{c}\right)^{2} T_{\text {seg }}^{2} \mathcal{R}^{-2}$. For the S5R3 and S5R5 runs, the nominal project duration was chosen to be 6 months. With the above choices, the search frequency ranges for S5R3 and S5R5 turn out to be respectively $[50,1200] \mathrm{Hz}$ and $[50,1190] \mathrm{Hz}$.

\section{Accuracy of spin-down model}

Let us briefly discuss the second-order spin-down $\ddot{f}$ which, as mentioned previously, is not a part of our search. For our frequency resolution $\delta f$, given by Eq. (9), and the full observation time $T_{\text {obs }}, \ddot{f}$ would have to be at least 


$$
\ddot{f}_{\min }=\delta f / T_{\mathrm{obs}}^{2} \approx 1.3 \times 10^{-20} \mathrm{~Hz} \mathrm{~s}^{-2}
$$

in order for the signal to move by a single frequency bin over the full observation time. On the other hand, for a minimum spin-down age $\tau_{\min }$ at a frequency $f$, a useful estimate for the range of $\ddot{f}$ that we should search is $f / \tau_{\text {min }}^{2}=\dot{f}_{\text {max }}^{2} / f$. Thus, $\ddot{f}$ is potentially more important at lower frequencies and higher spin-down values. Using the maximum value of $|\dot{f}|$ in the search and the minimum value of the search frequency gives us a value of $\ddot{f}$ that might be of astrophysical interest,

$\ddot{f}_{\text {ast }}=8 \times 10^{-20} \mathrm{~Hz} \mathrm{~s}^{-2}\left(\frac{|\dot{f}|_{\max }}{2.0 \times 10^{-9} \mathrm{~Hz} / \mathrm{s}}\right)^{2} \times\left(\frac{50 \mathrm{~Hz}}{f}\right)$.

Comparing with the minimum value of $\ddot{f}$ obtained above, we see that there is potentially a region in $(f, \dot{f})$ space where we could improve our astrophysical detection efficiency by including $\ddot{f}$; for our chosen range of $\dot{f}$ there is no effect of $\ddot{f}$ above $\sim 308 \mathrm{~Hz}$. It is important to note that the calculation of Eq. (36) is too conservative because it does not include any correlations between the phase evolution parameters. On the other hand, there is considerable uncertainty in the value of $\ddot{f}_{\text {ast }}$. If the neutron star has a braking index $n \equiv f \ddot{f} / \dot{f}^{2}$, then $\ddot{f}_{\text {ast }}$ increases by a factor $n$. If a star is spinning down purely due to gravitational wave emission, then $n=5$. On the other hand, for the Vela and Crab pulsars, observed values of $n$ are $\sim 1.4$ and $\sim 2.5$, respectively $[44,45]$.

The actual impact on our astrophysical reach is thus hard to quantify. Let us consider as an example the extreme case when the spin down is entirely due to gravitational radiation so that the braking index is $n=5$. For this case, a conservative estimate of the part of the spin-down range $|\dot{f}|_{\text {cons }}$ that is included in our search is

$$
\frac{-\dot{f}_{\text {cons }}}{2 \times 10^{-9} \mathrm{~Hz} \mathrm{~s}^{-1}} \leq 0.18 \sqrt{\frac{f}{50 \mathrm{~Hz}}} .
$$

Here we have used Eqs. (36) and (37) modified by the braking index factor. This corresponds to a minimum spindown value of $-1.8 \mathrm{nHz} \mathrm{s}^{-1}$ at the upper frequency of $1190 \mathrm{~Hz}$ and $-3.6 \times 10^{-10} \mathrm{~Hz} \mathrm{~s}^{-1}$ at $50 \mathrm{~Hz}$.

\section{S5R5 POSTPROCESSING}

As said earlier, roughly $10^{11}$ candidates from the S5R5 run were returned to the Einstein@Home server. They were then transferred to the 6720-CPU-core Atlas Computing Cluster [46] at the Albert Einstein Institute in Hannover and postprocessed. The goal is to filter the set of $10^{11}$ candidates, excluding false candidate events. The postprocessing strategy consists of the following steps:

(i) selection of 100 most significant candidates in $0.5 \mathrm{~Hz}$ frequency bands; (ii) removal of known instrumental noise artifacts;

(iii) removal of unknown data artifacts through the $\mathcal{F}$-statistic consistency veto;

(iv) follow-up of the most significant candidates with S5R3 data;

(v) fully coherent follow-up of the surviving candidates.

The items outlined above are described in the next subsections.

\section{A. Selecting the top candidates in frequency bands}

As is commonly done in CW searches, the results are examined separately in fixed-size search frequency bands; here we choose to perform the analysis in $0.5 \mathrm{~Hz}$ bands. As described earlier, in designing the WUs, we have previously been led to break up the frequency range in $\sim 20 \mathrm{mHz}$ bands and the sky has been partitioned as well. This was however done for purely technical reasons to make the search on Einstein@Home feasible. The choice of frequency bands for the postprocessing is based on different requirements. First, we would like the detector to have roughly constant sensitivity within each frequency band. Furthermore, as we shall see, the search does not result in a convincing detection candidate, and upper limits will be set over each of these frequency bands. Having a large number of very narrow bands would make the calculation of the upper limit very computationally intensive. The choice of $0.5 \mathrm{~Hz}$ is a compromise between these two requirements. This choice is in fact comparable to previous $\mathrm{CW}$ searches and will make comparisons straightforward. Finally, we note that all other things being equal, having a larger frequency band will in principle also lead to a decrease in sensitivity simply because of having a larger number of templates. This is however a relatively minor effect in the present case.

For each of the $0.5 \mathrm{~Hz}$-wide frequency bands, we select the 100 most significant candidates for further analysis, leading to a set of 228000 loudest S5R5 candidates. This choice was dictated by the available computational and human resources for the postprocessing. As will be illustrated in the following, at the end of the automated postprocessing procedure there will remain of order 10 candidates that survive all selection criteria. This is about the number that we can afford to follow-up manually with further investigations. As our follow-up procedures are further automated and optimized, it will become possible to consider lower thresholds and to inspect a correspondingly larger number of candidates.

The number count of such candidates is plotted in Fig. 3 as a function of the frequency. For most bands, it is consistent with expectations. The number count generally increases with increasing frequency because the number of sky points searched over increases [see Eq. (11)] and the maximum expected value of a random variable, over repeated trials, grows with the number of independent trials. 


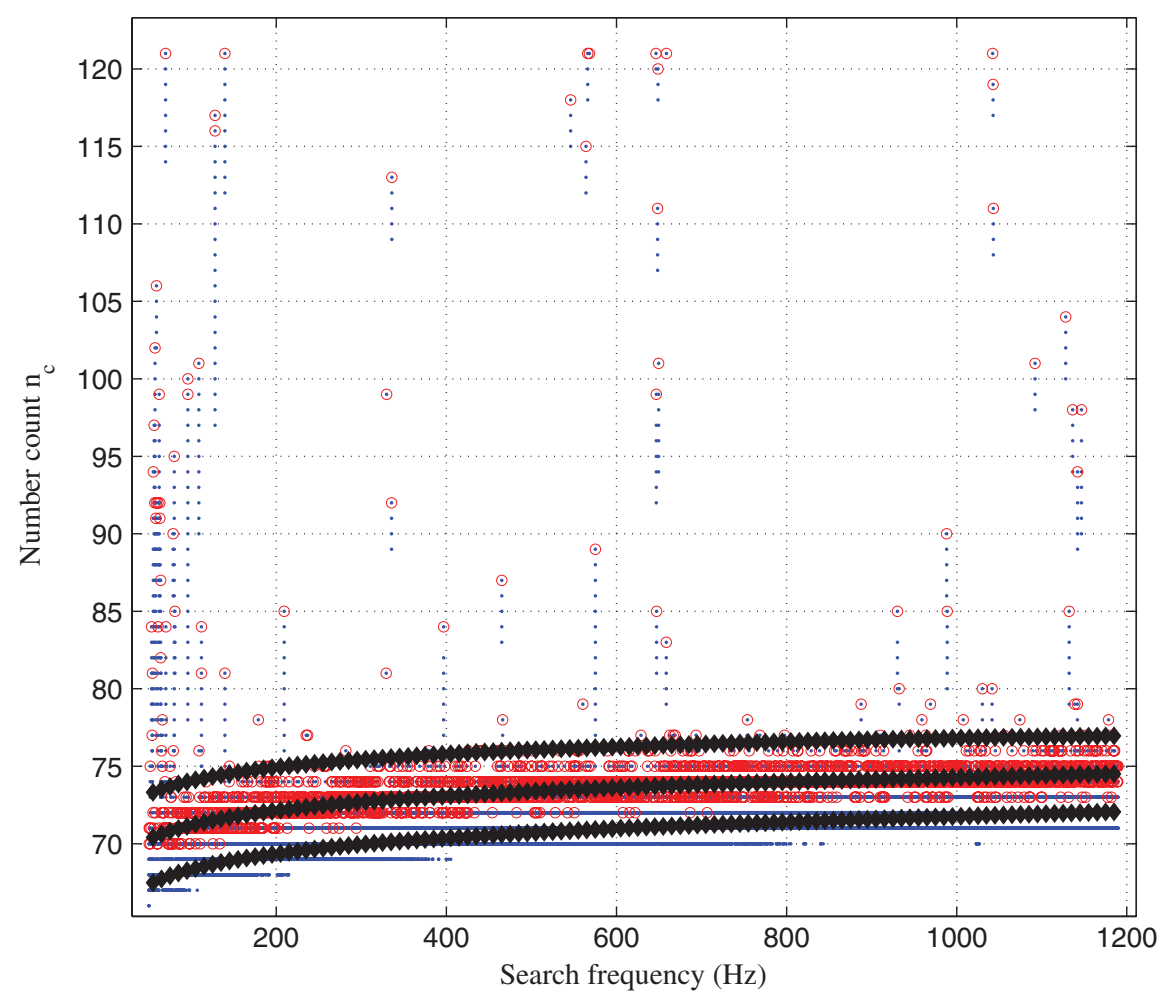

FIG. 3 (color). Number count of top 100 loudest candidates (blue dots) selected in $0.5 \mathrm{~Hz}$-wide frequency bands as a function of the search frequency, across the entire S5R5 search frequency range. The loudest (most significant) candidate in every $0.5 \mathrm{~Hz}$ band is indicated by a red circle. The expected values of the loudest candidates for Gaussian noise alone are shown by the central black curve. The lower and upper black curves show \pm 3 standard deviations from the expected value.

Let us ignore the effect of the weights, and take the Hough number count $n_{c}$ [defined in Eq. (17)] to be an integer random variable following a binomial distribution. The cumulative probability for obtaining $n_{c}$ or lower is

$$
F\left(n_{c}\right)=\sum_{n=0}^{n_{c}}\left(\begin{array}{c}
N_{\mathrm{seg}} \\
n
\end{array}\right) p_{\mathrm{seg}}^{n}\left(1-p_{\mathrm{seg}}\right)^{N_{\mathrm{seg}}-n}
$$

The binomial parameters are $N_{\text {seg }}=121$ and $p_{\text {seg }}=0.267$ (consistent with a $2 \mathcal{F}$ threshold of 5.2). The probability $p_{\max }$ that the maximum number count is $n_{c}$ over a set of $N_{\text {trials }}$ independent trials is

$$
p_{\text {max }}\left(n_{c}, N_{\text {trials }} \mid N_{\text {seg }}, p_{\text {seg }}\right)=F\left(n_{c}\right)^{N_{\text {trials }}}-F\left(n_{c}-1\right)^{N_{\text {trials }}} .
$$

We compute Eq. (40) as a function of $N_{\text {trials }}$, which we take to be the number of templates searched to cover $0.5 \mathrm{~Hz}$ bands, i.e., $7.5 \times 10^{4}$ frequency values $\times$ 18 spin-down values $\times 8444$ Hough pixels per $\mathcal{F}$ sky point $\times$ $N_{b}$, the total number of $\mathcal{F}$ sky points shown in Fig. 2. The number of Hough pixels has been computed using Eq. (14) with $m=0.3$ and $\wp=0.5$. Figure 3 shows the expected value of the maximum (central black curve), computed using the probability function given by Eq. (40), superimposed on our measurements (red circles) and confirming that there is broad agreement, in most bands, between our results and the expectations for Gaussian noise.

\section{B. Removing known data artifacts}

As a first step of the postprocessing pipeline, we eliminated from the list of top candidates any candidate whose frequency was too close to that of either a known artifact or to the cleaned noise bands described in Sec. III A. Specifically, we discarded those candidates whose detection statistic could have been constructed with contributions either from:

(i) data polluted in either of the two instruments by spurious disturbances; details of such detector disturbances are given in Appendix $\mathrm{C}$ and, in particular, a list of known spectral disturbances for the $\mathrm{H}$ and $\mathrm{L}$ instruments are listed in Tables VI and VII.

(ii) from fake noise that had been inserted by the cleaning process and hence could not host a CW signal (see Table I in Sec. III A).

After this veto, about $25 \%$ of the candidates were eliminated from the original set of 228000 loudest S5R5 candidates. More precisely, a total of 172038 S5R5 candidates survived this veto. The bandwidth removed due to the lines listed in Appendix C amounted to $\sim 243 \mathrm{~Hz}$; an additional $27 \mathrm{~Hz}$ was removed due to the cleaned noise bands. 


\section{The $\mathcal{F}$-statistic consistency veto}

We have thus far considered only known instrumental disturbances for vetoing candidates. However, we expect there to be more such disturbances present in the data that have not yet been explicitly identified. The idea is to discriminate between disturbances in a single detector and signals, which should produce consistent values of the $\mathcal{F}$-statistic in both detectors [47]. We refer to this method as the $\mathcal{F}$-statistic consistency veto.

For each of the 172038 S5R5 surviving candidates, the single-detector and multidetector $\mathcal{F}$-statistic was computed for each of the 121 data segments and then averaged over the segments. We refer to these averaged $2 \mathcal{F}$ values as $\left\langle 2 \mathcal{F}_{\mathrm{H}}\right\rangle$ and $\left\langle 2 \mathcal{F}_{\mathrm{L}}\right\rangle$ for the $\mathrm{H}$ and $\mathrm{L}$ detectors, respectively, and $\left\langle 2 \mathcal{F}_{\mathrm{HL}}\right\rangle$ for the coherent combination of the data from the two detectors. Candidates were discarded if either $\left\langle 2 \mathcal{F}_{\mathrm{H}}\right\rangle$ or $\left\langle 2 \mathcal{F}_{\mathrm{L}}\right\rangle$ were greater than $\left\langle 2 \mathcal{F}_{\mathrm{HL}}\right\rangle$. Using this veto, a small fraction $(4.1 \%)$ of candidates was eliminated, leaving 164971 surviving S5R5 candidates.

The impact of this veto is limited in this case due to the prior removal of the bulk of instrumental artifacts. However, the $\mathcal{F}$-statistic consistency veto represents an efficient method to remove disturbances that clearly stand out of the noise in the absence of independent instrumental evidence. Figure 4 shows the average $2 \mathcal{F}$ values for
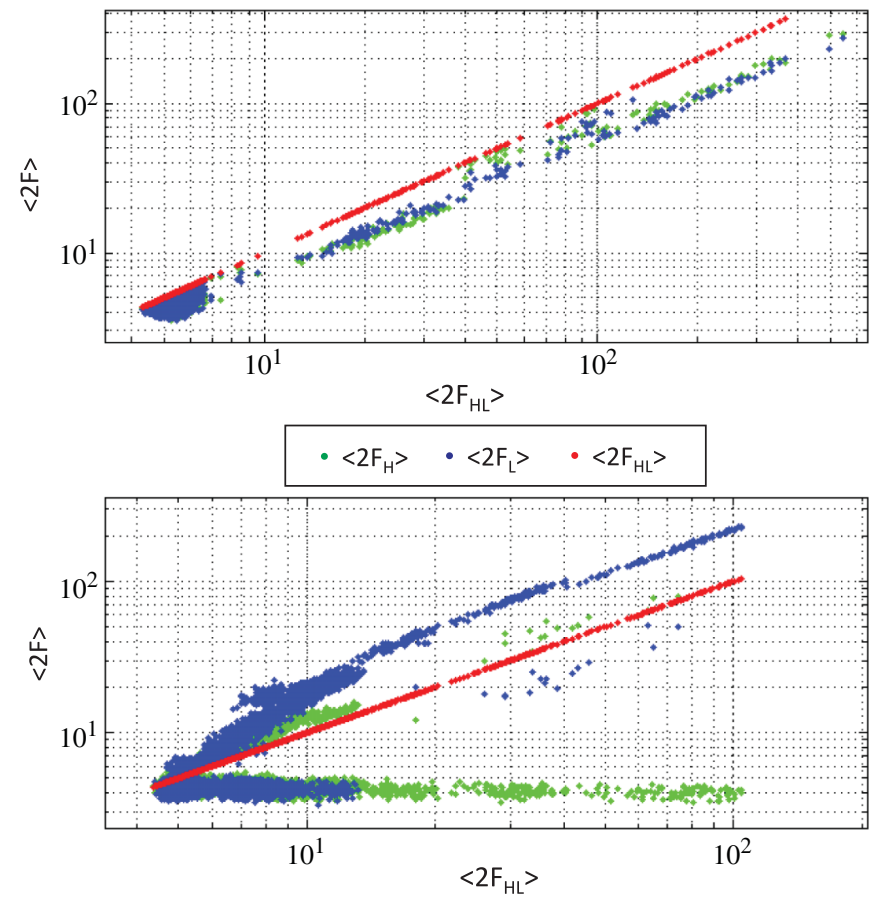

FIG. 4 (color). Values of $2 \mathcal{F}$ averaged over 121 data segments for the single-detector case, $\left\langle 2 \mathcal{F}_{\mathrm{H}}\right\rangle$ (green dots), $\left\langle 2 \mathcal{F}_{\mathrm{L}}\right\rangle$ (blue dots) and the multidetector case, $\left\langle 2 \mathcal{F}_{\mathrm{HL}}\right\rangle$ (red dots), against those for the combined multidetector statistic. The top (bottom) plot shows such values for 164971 (7067) surviving (vetoed) S5R5 candidates such that $\left\langle 2 \mathcal{F}_{\mathrm{H}}\right\rangle$ and (or) $\left\langle 2 \mathcal{F}_{\mathrm{L}}\right\rangle$ is less (greater) than $\left\langle 2 \mathcal{F}_{\mathrm{HL}}\right\rangle$.
164971 surviving (top plot) and 7067 vetoed (bottom plot) S5R5 candidates as a function of the multidetector average $2 \mathcal{F}$ values. By construction, all the surviving candidates in the top panel of Fig. 4 lie below the red dotted line, which defines the veto criterion.

\section{Distribution of candidates}

We have now applied all of our vetoes that try to remove instrumental artifacts. While there will of course remain other low amplitude instrumental spectral lines and hardware signal injections (described later in Sec. VI), we now need to deal with the possibility that, say, even Gaussian noise can mimic a signal in some cases. All remaining candidates will need to undergo detailed individual inspection and we will only be able to afford this for a few candidates. As our follow-up techniques become more refined, optimized and automated, we will be able to improve this part of the pipeline and dig deeper into the noise.

Figure 5 shows the histogram of $\left\langle 2 \mathcal{F}_{\mathrm{HL}}\right\rangle$ for the 164971 surviving candidates up to $\left\langle 2 \mathcal{F}_{\mathrm{HL}}\right\rangle$ values of 9 . The distribution actually extends up to $\left\langle 2 \mathcal{F}_{\mathrm{HL}}\right\rangle \sim 542.8$ but we show only the low $\left\langle 2 \mathcal{F}_{\mathrm{HL}}\right\rangle$-values distribution in order to explain our next choice of threshold. There are 166 candidates with $\left\langle 2 \mathcal{F}_{\mathrm{HL}}\right\rangle>9$ and they are all clustered at the two frequencies of $\sim 108.9 \mathrm{~Hz}$ and $\sim 575.2 \mathrm{~Hz}$, corresponding to two simulated signals injected in the data stream, as discussed in Sec. VI. The region $\left\langle 2 \mathcal{F}_{\mathrm{HL}}\right\rangle<6.5$ contains well over 99\% of the candidates and, as seen in Fig. 5, below 6.5 the density of candidates increases very sharply. We will take 6.5 as a threshold for the next step in our follow-up procedure.

The number of candidates expected to survive the $6.5 \mathrm{cut}$ on $\left\langle 2 \mathcal{F}_{\mathrm{HL}}\right\rangle$ in fixed $0.5 \mathrm{~Hz}$ bands increases with frequency because the number of sky locations searched scales with the square of the searched frequency. In order to compute

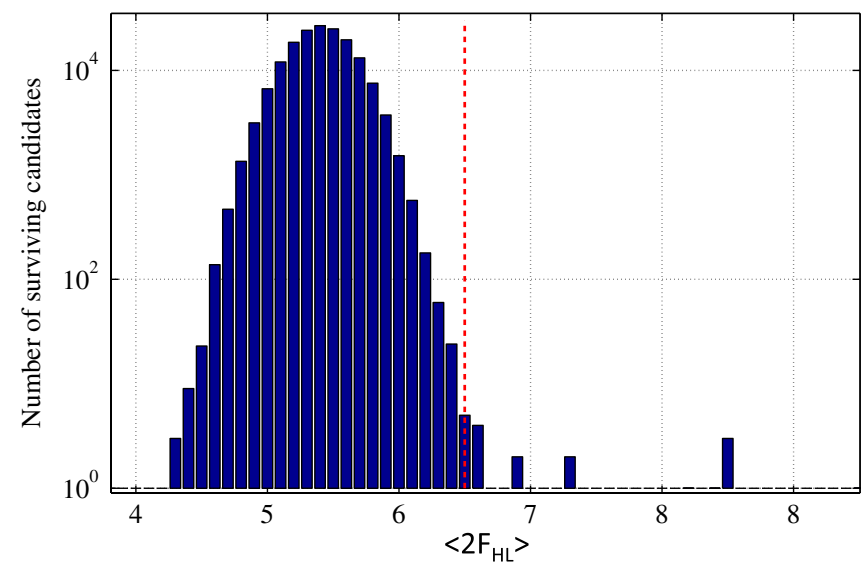

FIG. 5 (color online). Histogram of average multidetector $2 \mathcal{F}$ values for 164971S5R5 surviving candidates. The red dotted line draws the boundaries of the bulk of candidates due to instrumental noise and corresponds to the threshold $\left\langle 2 \mathcal{F}_{\mathrm{HL}}\right\rangle=6.5$. 

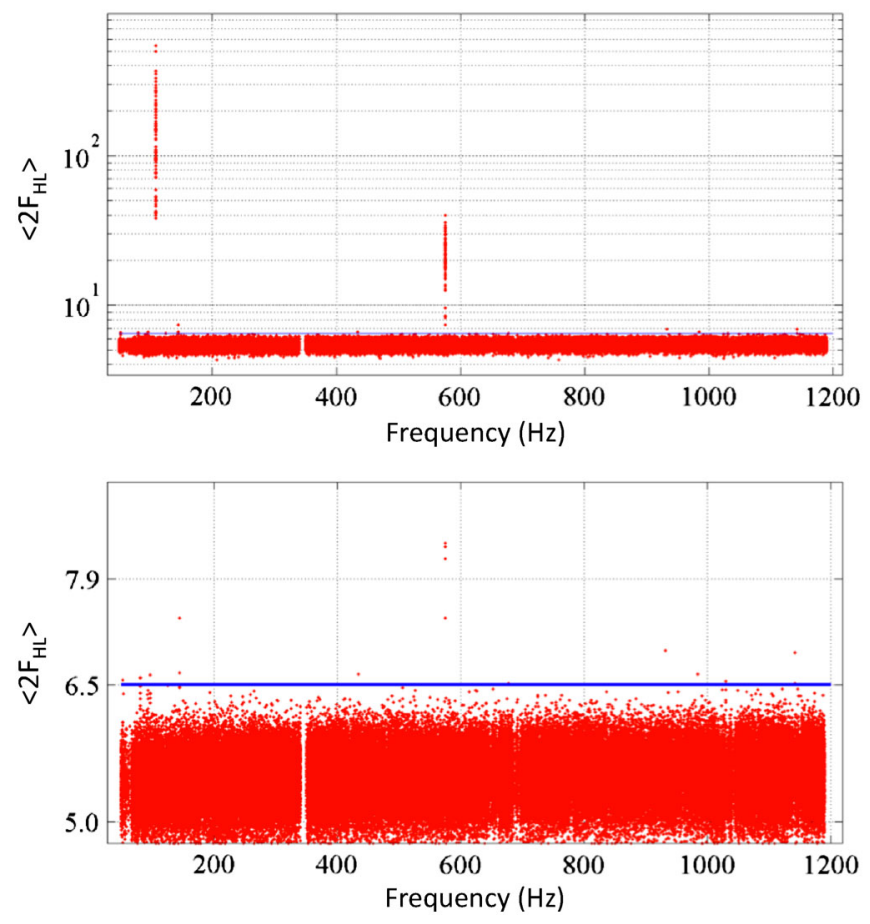

FIG. 6 (color online). Average multidetector $2 \mathcal{F}$ values of the S5R5 candidates surviving the $\mathcal{F}$-statistic consistency veto as a function of the frequency. The horizontal line represents the threshold value of $\left\langle 2 \mathcal{F}_{\mathrm{HL}}\right\rangle=6.5$. The bottom plot shows the top plot in the region close to the threshold.

the false alarm probability corresponding to this threshold in different frequency bands, we stress that in the absence of a signal, the value of $2 \mathcal{F}_{\mathrm{HL}}$ in the $i$ th segment, $2 \mathcal{F}_{\mathrm{HL}}^{(i)}$, follows a $\chi^{2}$ distribution with four degrees of freedom. Furthermore, since

$$
\left\langle 2 \mathcal{F}_{\mathrm{HL}}\right\rangle \times 121=\sum_{i=1}^{121} 2 \mathcal{F}_{\mathrm{HL}}^{(i)},
$$

it is clear that $\left\langle 2 \mathcal{F}_{\mathrm{HL}}\right\rangle \times 121$ is a $\chi^{2}$ random variable with $(4 \times 121)$ degrees of freedom. The false alarm probability corresponding to a threshold at $(6.5 \times 121)$ for such a random variable is $\sim 10^{-16}$. This corresponds to expected false alarm rates of about $0.1,0.6,2.6$, and $10 \%$ for searches in Gaussian noise over $0.5 \mathrm{~Hz}$ bands at 100 , 250, 500, and $1000 \mathrm{~Hz}$, respectively, considering the number of independent trials given by the number of searched templates in the respective bands. Disregarding the nonGaussian line features evident in Fig. 6, the ratio of the number of candidates observed above the 6.5 threshold at lower frequencies (say below $800 \mathrm{~Hz}$ ) to that at higher frequencies (say above $800 \mathrm{~Hz}$ ) is not inconsistent ${ }^{5}$ with the ratios of the false alarm rates computed above. We note that the false alarm probability given above overestimates

\footnotetext{
${ }^{5}$ Due to the low number statistics, it is hard to make a sharper statement.
}

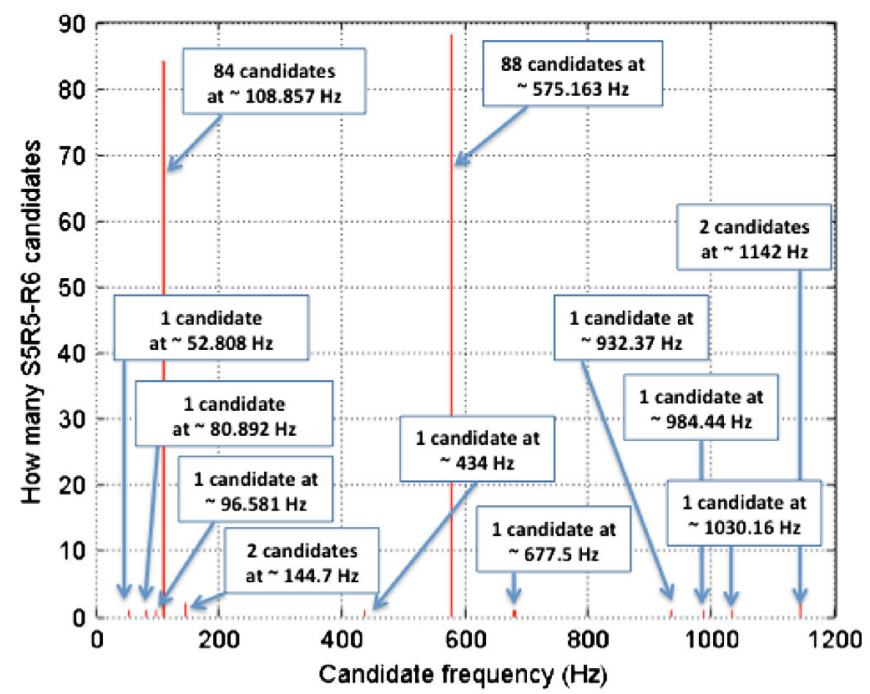

FIG. 7 (color online). Histogram of the S5R5 candidates that have been further investigated through deep follow-up study with the S5R3 data set.

the number of expected candidates above the 6.5 threshold because that threshold is not the only cut applied to the data. The previous cuts, discussed in the preceding sections, lower the actual false alarm probability of the surviving candidates.

There are 184 remaining S5R5 candidates, for which $\left\langle 2 \mathcal{F}_{\mathrm{HL}}\right\rangle>6.5$, and they are shown in Fig. 7 as a function of the frequency. They are clustered at twelve frequencies and only the most significant candidate from every cluster has been followed up.

\section{E. Following up candidates with S5R3 data}

For the next step in our postprocessing pipeline, recall that our underlying signal model of Eq. (1) assumes that the signal is long lived; thus its amplitude is constant in time and its intrinsic frequency evolves smoothly according to Eq. (5) with a constant spin down. This is an idealized model: although pulsars are the most stable clocks in the Universe, neutron stars are nonetheless known to glitch, to be perturbed by external agents, and in some cases to be affected by significant timing noise. Furthermore, for sufficiently long observation times, the spin-down evolution model that we use here, including only the first spin-down order, may not be adequate to describe the actual signal model (see also the discussion in Sec. III D). However, since the data set used in the S5R3 run ends just about a week before the S5R5 data set, it is reasonable to assume that any putative signals should be present in both data sets. Moreover, the average noise floor level in the detectors turns out to be approximately stable between S5R3 and S5R5. Thus, we might expect that a detectable signal should be visible in both searches.

The next follow-up step for each candidate then consists of a hierarchical search carried out on the same WU as 
TABLE II. S5R5 candidates followed up using the S5R3 data set. The different columns represent (I) the candidate frequency (shown also in Fig. 7), (II) its significance (CR), (III) the S5R5 number count $\left(N_{c}^{\mathrm{S} 5 \mathrm{R} 5}\right)$, (V) the expected, and (VI) observed number count values after following the candidate up $\left(E\left[N_{c}^{\mathrm{S} 5 \mathrm{R} 3}\right]\right.$ and $N_{c}^{\mathrm{S} 5 \mathrm{R} 3}$, respectively).

\begin{tabular}{lrrcc}
\hline \hline Frequency $(\mathrm{Hz})$ & \multicolumn{1}{c}{$\mathrm{CR}$} & $N_{c}^{\mathrm{S} 5 \mathrm{R} 5}$ & $E\left[N_{c}^{\mathrm{S} 5 \mathrm{R} 3}\right]$ & $N_{c}^{\mathrm{S} 5 \mathrm{R} 5}$ \\
\hline 52.808297682 & 7.7 & 70 & 49 & 37 \\
80.891816186 & 10.1 & 82 & 57 & 34 \\
96.581099597 & 8.1 & 72 & 50 & 37 \\
108.85716501 & 13.9 & 101 & 70 & 55 \\
144.74321811 & 7.9 & 71 & 49 & 42 \\
434.09886421 & 7.7 & 70 & 49 & 46 \\
575.16357663 & 11.6 & 89 & 62 & 53 \\
677.47882796 & 8.0 & 71 & 49 & 46 \\
932.36948703 & 8.5 & 74 & 51 & 46 \\
984.44286823 & 8.2 & 73 & 51 & 47 \\
1030.1650892 & 9.1 & 77 & 53 & 53 \\
1141.9926498 & 9.4 & 78 & 54 & 48 \\
\hline \hline
\end{tabular}

done for S5R5 (i.e., over the same parameter space), but using the S5R3 data set. The closest ${ }^{6}$ candidate to the original one from such a search was identified and the value of its detection statistic was compared with what one would expect if the S5R5 candidate were due to a signal. In particular, the expected number counts in S5R3 and S5R5 should be related according to

$$
E\left[N_{c}^{\mathrm{S} 5 \mathrm{R} 3}\right]=N_{c}^{\mathrm{S} 5 \mathrm{R} 5} \times 84 / 121,
$$

where 84 is the number of data segments used in S5R3, as we shall see in Appendix B. Possible reasons for this not to be a good approximation would be if the detector noise floor were to vary significantly between S5R3 and S5R5 or the fact that the relative geometry between the detector and source varies in time. We have already remarked that the noise floor is, on the average, stable between S5R3 and S5R5. Furthermore, albeit in each segment the expected $2 \mathcal{F}$ values for a given signal might be different, if we average this expected value over many nonoverlapping segments, we expect this to converge within a few tens of segments; recall here that each segment spans a duration of 25 hours, while the antenna pattern function of the detectors has a periodicity of 24 hours. Thus, it is reasonable to assume that Eq. (42) is valid.

Candidates for which the measured value $N_{c}^{\mathrm{S} 5 \mathrm{R} 3}$ was more than $3 \sigma$ less significant than the expected $E\left[N_{c}^{\mathrm{S} 5 \mathrm{R} 3}\right]$ were discarded as not being consistent with a $\mathrm{CW}$ signal, where $\sigma$ was computed using Eq. (22). As shown in Table II, two candidates were discarded by this follow-up test, at $\sim 80.9 \mathrm{~Hz}$ and $\sim 108.9 \mathrm{~Hz}$. However, the second of

\footnotetext{
${ }^{6}$ The distance used to judge closeness between candidates is a Euclidean distance expressed in bins in the four dimensions $(f, \dot{f}, \alpha, \delta)$.
}

these, as well as the candidates at $\sim 52.8 \mathrm{~Hz}$ and $\sim 575.2 \mathrm{~Hz}$, represent three simulated signals injected only part of the time during S5, as discussed in Sec. VI.

As we can see from Fig. 7, the bulk of the candidates arise from the strong hardware-injected pulsar 2 and 3 signals (see Sec. VI). In particular, 84 and 88 candidates, respectively, are clustered near the frequencies of these two injected signals.

\section{F. Fully coherent follow-up}

Excluding the hardware-injected simulated signals, the postprocessing up to this point has left us with eight surviving candidates. These have been significant enough to pass our thresholds and have not been clearly identified as instrumental artifacts, or eliminated by inconsistency between the $\mathrm{H}$ and $\mathrm{L}$ detectors, or by the follow-up with the S5R3 data set. We therefore need to consider other more sensitive methods. If these candidates are real signals, then their SNRs and significance should increase if the parameter space grids are made finer, or as the coherent integration time becomes larger.

We use a three-step procedure consisting of a grid-based semicoherent Hough search, followed by a semicoherent and a final fully coherent $\mathcal{F}$-statistic search, using the Mesh Adaptive Direct Search (MADS) algorithm for constrained optimization. The reference implementation of the MADS algorithm is publicly available through the NOMAD library [48,49]. Hence, in the following, we refer to such searches simply as NOMAD searches. Contrary to the traditional grid-based methods, a mesh adaptive search constructs the trial points as the search evolves aiming to find the maximum of the statistic.

The three steps of the follow-up procedure are the following:

(1) Re-run the Hough search around a given candidate, but with a finer grid to reduce the mismatch with a putative signal. The search region includes five frequency bins on either side around the candidate and 16 neighboring coarse sky-grid points. The fine Hough sky grid is refined by a factor of 2 in each direction by using $\wp=1$ (see Eq. (13)) instead of 0.5 as in the original search. Furthermore, we refine the coarse $\dot{f}$ grid spacing of Eq. (10) by a factor $N_{\text {seg }}=121$ [19].

(2) The loudest candidate from the first step is used as a starting point for the semicoherent $\mathcal{F}$-statistic NOMAD optimization. The detection statistic in this step is the sum of the $\mathcal{F}$-statistic values from each segment. This search has been performed in a fixed parameter space box around the starting point. The dimensions of the box are $\Delta f=10^{-4} \mathrm{~Hz}$, $\Delta \alpha=0.10 \mathrm{rad}, \quad \Delta \delta=0.24 \mathrm{rad}$ and $\Delta \dot{f}=$ $10^{-10} \mathrm{~Hz} \mathrm{~s}^{-1}$. The loudest candidate found in this semicoherent $\mathcal{F}$-statistic NOMAD search is passed on to the next step. 
(3) In the third step, the loudest candidate from the previous step is used as a starting point for the fully coherent $\mathcal{F}$-statistic NOMAD search. This search spans the entire duration of the S5R5 data set and has been carried out in a parameter space box defined by using the diagonal elements of the inverse Fisher matrix in each dimension around the starting point. These elements are described by Eq. (16) in Ref. [50] and have been computed from the inverse of the semicoherent parameter space metric computed at the candidate point, rescaled by the measured SNR at the same point; for more details we refer the reader to Ref. [50].

In both the semicoherent and fully coherent NOMAD searches, we ran multiple instances of the algorithm iterating over the mesh coarsening exponent using both deterministic [48] and stochastic [51] methods for the choice of search directions. Based on Monte-Carlo studies, the falsedismissal probability of the follow-up procedure is found to be less than $10 \%$.

As said earlier, in the presence of a real signal we expect the significance of a candidate to increase as the template grid becomes finer because there will be a template with a smaller mismatch with respect to the real signal. At that template the signature of the signal should be more evident and all the consistency tests should continue to hold. If the candidate signal detected on the finer grid does not pass a consistency test, this indicates that it is not behaving as we would expect from the signals that we are targeting. The candidates at $\sim 96.6 \mathrm{~Hz}, \sim 144.7 \mathrm{~Hz}, \sim 932.4 \mathrm{~Hz}, \sim 1030.2 \mathrm{~Hz}$ and $\sim 1142 \mathrm{~Hz}$ fail a multidetector versus single-detector $\mathcal{F}$-statistic consistency test (see Sec. IV C) after performing the semicoherent NOMAD search; therefore, they cannot be considered defensible CW signals. Moreover, line artifacts appear in the average power spectrum of $\mathrm{S} 5 \mathrm{H}$ data at $\sim 932.4 \mathrm{~Hz}, \sim 1030.2 \mathrm{~Hz}$ and $\sim 1142 \mathrm{~Hz}$.

The remaining candidates, namely at $\sim 434.1 \mathrm{~Hz}$, $\sim 677.5 \mathrm{~Hz}$ and $\sim 984.4 \mathrm{~Hz}$, survive the $\mathcal{F}$-statistic consistency test on the finer grid and are followed up with the fully coherent $\mathcal{F}$-statistic NOMAD search. However, for each of them, the maximum value of the detection statistic over the parameter space searched is much lower than would be expected based on the original candidate parameters, and in fact is consistent with the expectation for Gaussian noise. Hence, also these three candidates do not survive a more sensitive inspection and cannot be considered viable detection candidates. Thus, we see that all the candidates listed in Table II are inconsistent with the properties of a true $\mathrm{CW}$ signal.

\section{UPPER LIMIT ESTIMATION}

The analysis of the Einstein@Home searches presented here has not identified any convincing CW signal. Hence, we proceed to set upper limits on the maximum intrinsic gravitational wave strain $h_{0}$ that is consistent with our observations for a population of $\mathrm{CW}$ signals described by Eq. (8), from random positions in the sky, in the gravitational wave frequency range $[50.5,1190] \mathrm{Hz}$, and with spin-down values in the range of $\sim[-20,1.1] \times$ $10^{-10} \mathrm{~Hz} \mathrm{~s}^{-1}$. The nuisance parameters $\cos \iota, \phi_{0}$, and $\psi$ are assumed to be uniformly distributed. As commonly done in all-sky, all-frequency searches, the upper limits are given in different frequency sub-bands and here we have chosen these to be $0.5 \mathrm{~Hz}$ wide. Each upper limit is based on the most significant event from the S5R5 search in its $0.5 \mathrm{~Hz}$ band.

\section{A. Monte-Carlo upper-limit estimates}

Our procedure for setting upper limits uses Monte-Carlo signal injection studies using the same search and postprocessing pipeline (except for the S5R3 and fully coherent follow-ups) that we have described above. In every $0.5 \mathrm{~Hz}$ band, our goal is to find the value of $h_{0}$ (denoted $h_{0}^{90 \%}$ ) such that $90 \%$ of the signal injections at this amplitude would be recovered by our search and are more significant than the most significant candidate from our actual search in that band. We can thus exclude, with $90 \%$ confidence, the existence of sources (from our specific population) that have an amplitude $h_{0}>h_{0}^{90 \%}$.

In order to estimate $h_{0}^{90 \%}$, for each injection at a randomly chosen parameter space point, a hierarchical search is performed over a small parameter space region, which consists of

(i) a $0.8 \mathrm{mHz}$ frequency band centered at the S5R5 frequency grid point closest to the randomly chosen source frequency;

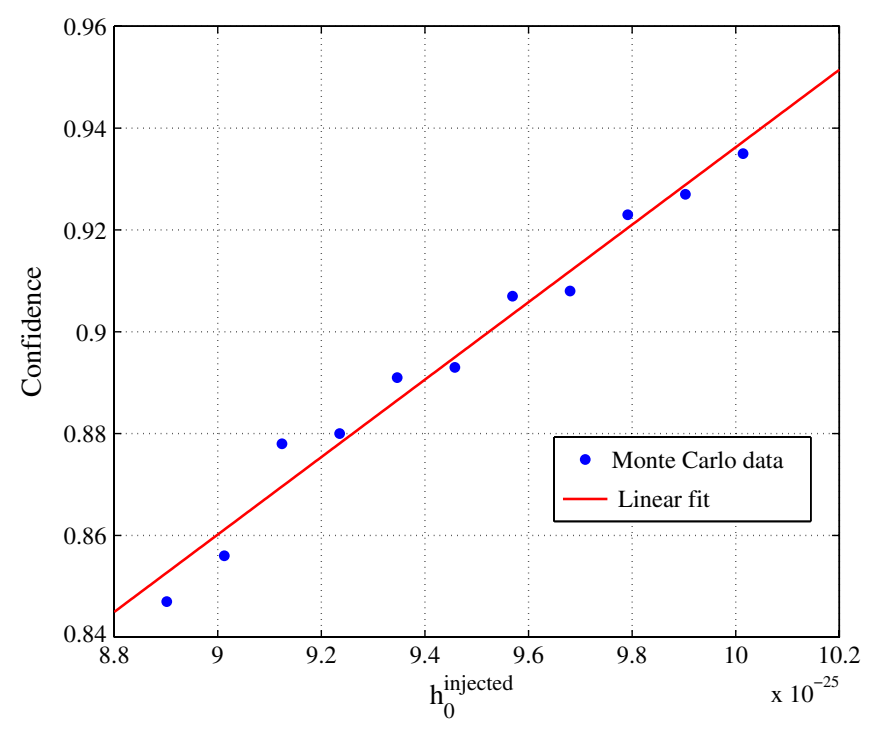

FIG. 8 (color online). Confidence versus the injected $h_{0}$ values for sets of 1000 injections in the band [216, 216.5] Hz. This plot illustrates how the uncertainty on the confidence level affects the uncertainty on the value of $h_{0}^{90 \%}$. 
(ii) four spin-down values around the S5R5 frequency derivative grid point closest to the randomly chosen spin down;

(iii) a sky patch consisting of 10 S5R5 coarse sky-grid points closest to the randomly chosen sky location.

At the end of each hierarchical search, the most significant candidate is selected and postprocessed as described in the previous section. The vetoes for excluding known instrumental lines are not required because we have already excluded them from the upper-limit analysis. We first check if this candidate is significant enough to be part of the 100 Einstein@Home loudest candidates originally selected in its corresponding $0.5 \mathrm{~Hz}$ band. Then we perform the $\mathcal{F}$-statistic consistency check and finally we compare the computed average multiinterferometer $2 \mathcal{F}$ value $\left(\left\langle 2 \mathcal{F}_{\mathrm{HL}}^{\text {Cand }}\right\rangle\right)$ with the maximum $\left\langle 2 \mathcal{F}_{\mathrm{HL}}\right\rangle$ value we have in the corresponding $0.5 \mathrm{~Hz}$ band. If $\left\langle 2 \mathcal{F}_{\mathrm{HL}}^{\text {Cand }}\right\rangle$ is greater than the maximum $\left\langle 2 \mathcal{F}_{\mathrm{HL}}\right\rangle$, then the simulated source is considered to be recovered and more significant than the most significant candidate of the search. The confidence level is defined as $C=n_{\text {rec }} / n_{\text {tot }}$, where $n_{\text {rec }}$ is the number of recovered candidates, and $n_{\text {tot }}=100$ is the total number of injections performed.

After some preliminary tuning to determine a range of $h_{0}$ values close to the $90 \%$ confidence level, we use an iterative procedure to determine the confidence as a function of the injected population $h_{0}$ until we hit a confidence value close to $90 \%$, within the expected $1 \sigma$ fluctuations. Since we use 100 injections, from a binomial statistic we estimate the $1 \sigma$ fluctuation to be $3 \%$ and hence we associate the $h_{0}^{90 \%}$ value to any measured confidence in the range $87 \%-93 \%$. The $3 \%$ uncertainty in confidence translates to an uncertainty in $h_{0}^{90 \%}$ smaller than $5 \%$, as can be seen from Fig. 8, which shows a typical confidence versus injected $h_{0}$ behavior. Each point in Fig. 8 was derived with

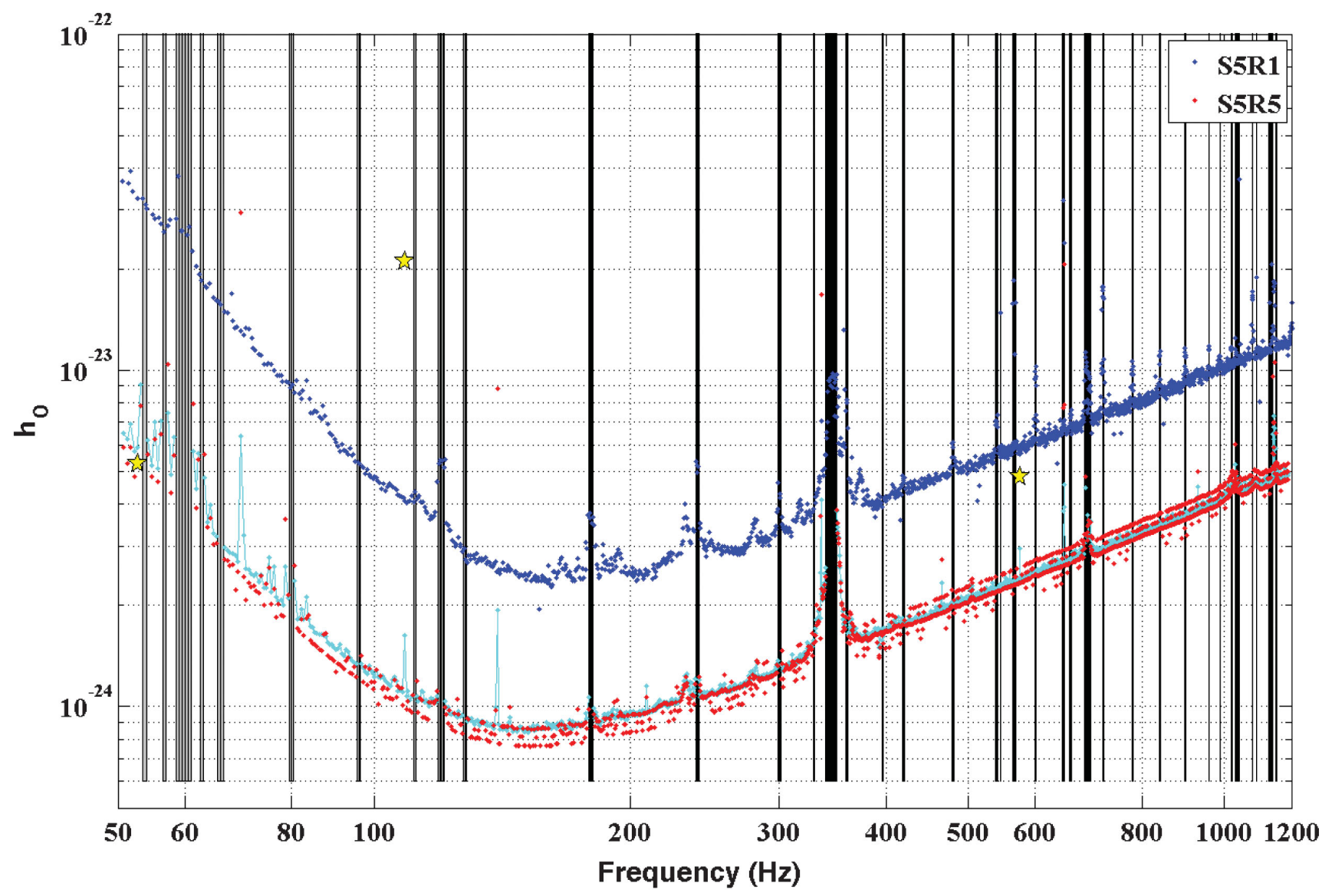

FIG. 9 (color). Upper limits for the S5R5 Einstein@Home search (red dots) as well as the previous Einstein@ Home search, called S5R1, which used early S5 data (blue dots) [24]. The three stars correspond to hardware-injected simulated pulsars which were recovered in the S5R5 search. The curves represent the source strain amplitude $h_{0}$ at which $90 \%$ of simulated signals would be detected. The vertical bars represent 156 half-Hz frequency bands contaminated by instrumental disturbances for which no upper limits are provided. The upper limits for the $0.5 \mathrm{~Hz}$-wide bands starting at $69.5,139.5,335.5$ and $648 \mathrm{~Hz}$ are fairly high due to significant partial contamination in these bands by lines listed in Tables VI and VII. Note that the broadness of the red curve is due to the 5\% steps used to vary the injected population $h_{0}$ values in the Monte-Carlo signal software-injections until a confidence value close to $90 \%$ is reached. In addition, less than 1/4 of the spectral range shown was excluded in many narrow bands because of known instrumental artifacts, as described in Sec. IV B. The cyan curve shows the predicted $h_{0}^{90 \%}$ upper limits according to Eq. (43). 
1000 injections and hence is affected by fluctuations smaller than $1 \%$.

The lower (red) curve in Fig. 9 shows the resulting upper limits as a function of the frequency. The upper (blue) curve shows the upper limit values from the previous Einstein@Home search in early S5 data [24]. The current upper limit values are about a factor 3 more constraining than the previous Einstein@Home ones. In particular, the most constraining upper limit falls in the $0.5 \mathrm{~Hz}$-wide band at $152.5 \mathrm{~Hz}$, where we can exclude the presence of signals with $h_{0}$ greater than $7.6 \times 10^{-25}$. The three stars shown in Fig. 9 correspond to the simulated pulsars 2, 3, and 5, i.e., the hardware injections recovered in the S5R5 search (discussed in Sec. VI).

The numerical data for the plot in Fig. 9 can be obtained separately [52]. A conservative estimate of the overall uncertainty on the $h_{0}^{90 \%}$ values shown in Fig. 9 is $15 \%$, having added to the $1 \sigma$ statistical upper limit estimation procedure uncertainty the $10 \%$ amplitude calibration uncertainties for the data used in this Einstein@Home run [53].

As we have excluded from the search those frequency bands hosting spectral artifacts (Tables VI and VII) and the cleaned noise bands (Table I), we therefore also exclude these frequency intervals from the upper limit statements. Vertical bars in Fig. 9 represent 156 half-Hz frequency bands for which no upper limits are provided because the entire half-Hz band has been excluded.

As shown in Fig. 9, the upper limits on $h_{0}$ provided in the $0.5 \mathrm{~Hz}$-wide bands starting at 69.5, 139.5, 335.5, and $648 \mathrm{~Hz}$ are fairly high, roughly equal to $3 \times 10^{-23}$, $8.8 \times 10^{-24}, 1.7 \times 10^{-23}$ and $2 \times 10^{-23}$, respectively. This is due to significant partial contamination in these bands by lines listed in Tables VI and VII; the upper limit is given for the remaining, clean part of the band, but loud candidates from the disturbed part make up the loudest 100 candidates selected in the processing, so a simulated signal must be especially loud to surpass those. Note that, for the same reason, if we had set upper limits in the 156 half- $\mathrm{Hz}$ bands shown in Fig. 9 we would have obtained similarly high upper limits on $h_{0}$.

\section{B. Analytic sensitivity estimates}

The $h_{0}^{90 \%}$ upper limits can be independently predicted using the method in Ref. [54], adapted to the Houghon- $\mathcal{F}$-statistic search method (see Ref. [55] for details). The upper limit procedure described above is modeled by a simple threshold on the number count, where the thresholds are the largest number counts observed in each $0.5 \mathrm{~Hz}$ upper limit band. The probability that, in the neighborhood of an injected signal, the number count $n_{\mathrm{c}}$ will exceed a threshold $n_{\mathrm{c}, \text { th }}$ is denoted by $P\left[n_{\mathrm{c}}>\right.$ $\left.n_{\mathrm{c}, \text { th }} \mid \rho\left(h_{0}, \cos \iota, \phi_{0}, \psi, m\right)\right]$; this probability can be calculated analytically from the known distribution of $n_{\mathrm{c}}$. The recovered $\mathrm{SNR}, \rho$, is a function of the nuisance parameters and of the mismatch $m$ between the injected signal and the nearest template. In addition, note that in the presence of a signal, $2 \mathcal{F}$ follows a non-central $\chi^{2}$ distribution with 4 degrees of freedom; $\rho^{2}$ is the noncentrality parameter of this distribution. In the presence of a signal, averaging $P$ over the parameters of $\rho$ (except $\left.h_{0}\right)$ gives $\left\langle P\left(n_{\mathrm{c}}\right\rangle\right.$ $\left.\left.n_{\mathrm{c}, \mathrm{th}} \mid \rho\left(h_{0}\right)\right)\right\rangle$, which equals the confidence of recovering a population of injections with amplitude $h_{0}$. In each $0.5 \mathrm{~Hz}$ band, we determine the value of $h_{0}$ such that $\left\langle P\left(n_{\mathrm{c}}\right\rangle\right.$ $\left.\left.n_{\mathrm{c}, \text { th }} \mid \rho\left(h_{0}\right)\right)\right\rangle=90 \%$; this value is then the predicted value of $h_{0}^{90 \%}$, given by

$$
h_{0}^{90 \%}=H \sqrt{\frac{S_{h}}{T_{\text {data }}}}
$$

as a function of the detector noise $S_{h}$ and the total data volume $T_{\text {data }}=N_{\text {seg }} T_{\text {seg }}$. The factor $H$ varies from $\sim 141$ to
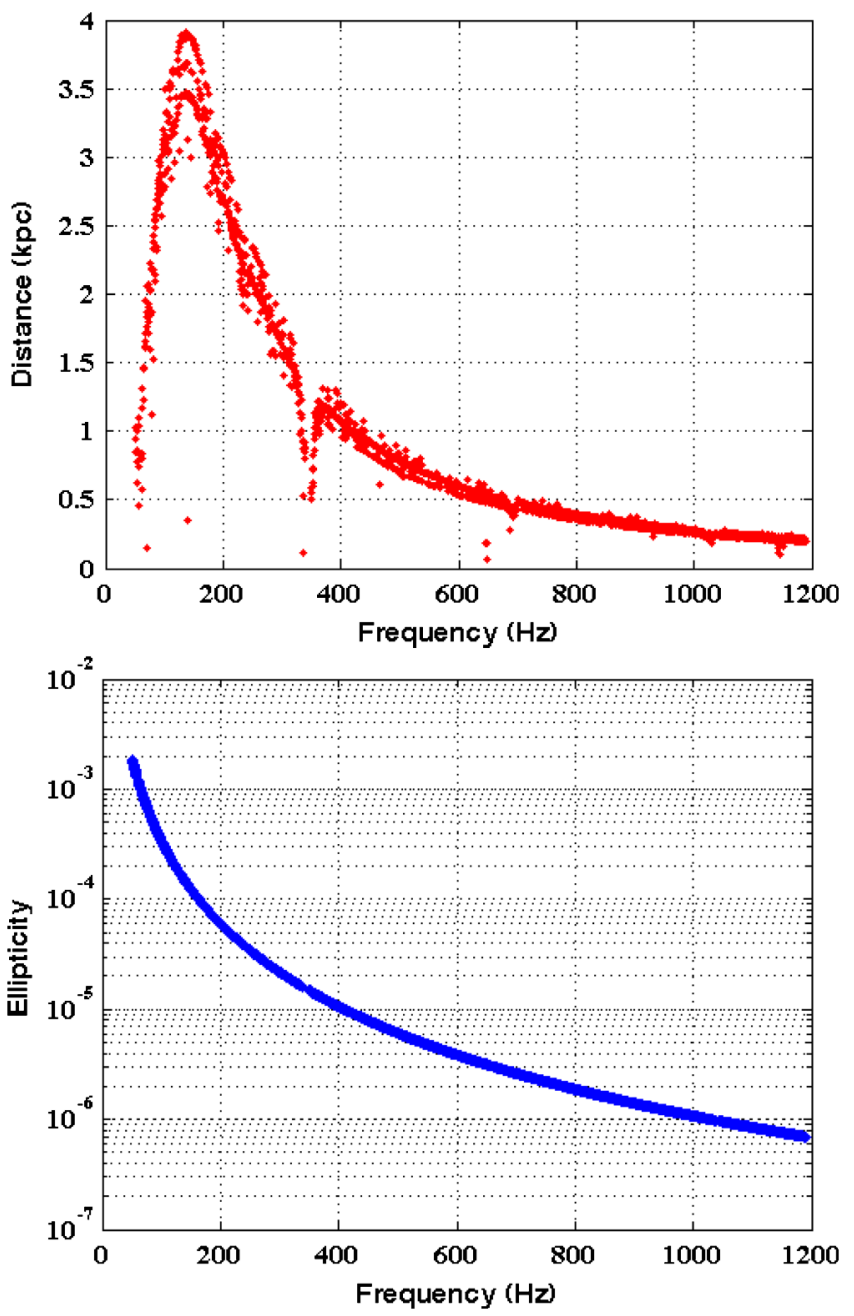

FIG. 10 (color online). Panel (a) and (b) represent the distance range (in $\mathrm{kpc}$ ) and the maximum ellipticity, respectively, as a function of the frequency. Both the panels are valid for neutron stars spinning down solely due to gravitational radiation and assuming a spin-down value of $\sim-2 \mathrm{nHz} \mathrm{s}^{-1}$. In these plots, the 156 half-Hz frequency bands for which no upper limits are provided have not been considered. 
$\sim 150$ over the range of search frequencies, and is plotted in Ref. [55]. It is given by $H=2.5 \hat{\rho}^{90 \%} \sqrt{N_{\text {seg }}}$, where $\hat{\rho}^{90 \%}$ is the mean injected SNR per segment of a population of signals as described above, and is itself a function of the false alarm and false dismissal probabilities, and $N_{\text {seg }}$ [54,55]. The variation of $H$ as a function of frequency arises from the variation of $\hat{\rho}^{90 \%}$ as a function of the false alarm probability in each upper limit band, which are calculated from the largest number counts $n_{\mathrm{c}}$ plotted in Fig. 3.

The predicted values are shown by the cyan curve in Fig. 9. The root-mean-square error between the MonteCarlo estimated and predicted $h_{0}^{90 \%}$ values $(\sim 7 \%$ over all frequencies) is comparable with the uncertainties due to calibration $(10 \%)$ and the finite stepping of the Monte Carlo procedure (5\%). This demonstrates that the sensitivity of the Hough-on- $\mathcal{F}$-statistic search method, as a function of the search parameters, is well understood.

\section{Astrophysical reach}

Figure 10 shows the maximum reach of our search. The top panel shows the maximum distance at which we could have detected a source emitting a $\mathrm{CW}$ signal with strain amplitude $h_{0}^{90 \%}$. The source is assumed to be spinning down at the maximum rate considered in the search, $\sim-2 \mathrm{nHz} \mathrm{s}^{-1}$, and emitting at the spin-down limit, i.e., with all of the lost rotational energy going into gravitational waves. The intrinsic gravitational wave strain from a source at a distance $d$, with frequency $f$, frequency derivative $\dot{f}$, and emitting at the spin-down limit is

$$
h_{0}^{\mathrm{sd}}=4.54 \times 10^{-24}\left(\frac{1 \mathrm{kpc}}{d}\right)\left(\frac{250 \mathrm{yr}}{-f /(4 \dot{f})}\right)^{1 / 2},
$$

where the canonical value of $10^{38} \mathrm{~kg} \mathrm{~m}^{2}$ is assumed for $I_{z z}$ in Eq. (4). The bottom panel of the figure does not depend on any result from the search. It shows the spin-down ellipticity values as a function of the frequency for sources emitting in gravitational waves all the energy lost while spinning down at a rate of $\sim-2 \mathrm{nHz} \mathrm{s}^{-1}$. This is obtained by setting $\dot{f}=-2 \mathrm{nHz} \mathrm{s}^{-1}$ in the following equation:

$\varepsilon^{\mathrm{sd}}=7.63 \times 10^{-5}\left(\frac{-\dot{f}}{10^{-10} \mathrm{~Hz} \mathrm{~s}^{-1}}\right)^{1 / 2}\left(\frac{100 \mathrm{~Hz}}{f}\right)^{5 / 2}$.

Around the frequency of greatest sensitivity, $152.5 \mathrm{~Hz}$, we are sensitive to objects as far as $3.8 \mathrm{kpc}$ and with an ellipticity $\varepsilon \sim 10^{-4}$. Normal neutron stars are expected to have $\varepsilon$ less than a few times $10^{-6}$ based on theoretical predictions [31]. A plausible value of $\varepsilon \sim 3.5 \times 10^{-6}$ could be detectable by a search like this if the object were emitting at $625 \mathrm{~Hz}$ and at a distance no further than $500 \mathrm{pc}$.

\section{STUDY OF HARDWARE-INJECTED SIGNALS}

As part of the testing and validation of search pipelines and analysis codes, simulated signals are added into the interferometer length control system to produce mirror motions similar to what would be generated if a gravitational wave signal were present. Table III shows the parameters of the set of simulated $\mathrm{CW}$ signals injected into the LIGO detectors; we shall often refer to these injections also as "fake pulsars." These injections were active from the GPS epoch 829412600 s until 875301345 s. Of these ten hardware-injected CW signals, eight had frequencies covered by the S5R5 search frequency band: the fake pulsars 4 and 7 have frequencies outside this band and thus have not been taken into account during this analysis. The fake pulsars 6 and 8 have spin-down values outside the S5R5 search frequency derivative range.

As a minor complication, the hardware injections were not active all the time. In the S5R5 data set, their duty cycle was $\sim 63 \%$ and $\sim 60 \%$ in $\mathrm{L}$ and $\mathrm{H}$, respectively. The hardware injections were active in 76 of the 121 S5R5

TABLE III. Simulated ("fake") pulsar hardware injections during the S5 LIGO run, created with the JPL DE405 Sun and Earth ephemeris files. The pulsar parameters are defined at the GPS reference time of $751680013 \mathrm{~s}$ in the SSB frame. These are the frequency $f_{P}$, the spin down $\dot{f}$, the sky position $(\alpha, \delta)$, the polarization angle $\psi$, the initial phase $\phi_{0}$, the inclination parameter cos $\iota$ and the dimensionless strain amplitude $h_{0}$. These parameters correspond to the only set of hardware injections, injected into the S5 LIGO data, that fall within the GPS times of the S5R5 data.

\begin{tabular}{lcccrrrrr}
\hline \hline Name & $f_{P}(\mathrm{~Hz})$ & $\dot{f}\left(\mathrm{~Hz} \mathrm{~s}^{-1}\right)$ & $\alpha(\mathrm{rad})$ & \multicolumn{1}{c}{$\delta(\mathrm{rad})$} & \multicolumn{1}{c}{$\psi(\mathrm{rad})$} & \multicolumn{1}{c}{$\phi_{0}(\mathrm{rad})$} & \multicolumn{1}{c}{$\cos \iota(\mathrm{rad})$} & $h_{0}$ \\
\hline Fake pulsar 0 & 265.5771052 & $-4.15 \times 10^{-12}$ & 1.248817 & -0.981180 & 0.770087 & 2.66 & 0.794905 & $2.47 \times 10^{-25}$ \\
Fake pulsar 1 & 849.0832962 & $-3.00 \times 10^{-10}$ & 0.652646 & -0.514042 & 0.356036 & 1.28 & 0.463822 & $1.06 \times 10^{-24}$ \\
Fake pulsar 2 & 575.163573 & $-1.37 \times 10^{-13}$ & 3.756929 & 0.060109 & -0.221788 & 4.03 & -0.928576 & $4.02 \times 10^{-24}$ \\
Fake pulsar 3 & 108.8571594 & $-1.46 \times 10^{-17}$ & 3.113189 & -0.583579 & 0.444280 & 5.53 & -0.080666 & $1.63 \times 10^{-23}$ \\
Fake pulsar 4 & 1403.163331 & $-2.54 \times 10^{-8}$ & 4.886707 & -0.217584 & -0.647939 & 4.83 & 0.277321 & $4.56 \times 10^{-23}$ \\
Fake pulsar 5 & 52.80832436 & $-4.03 \times 10^{-18}$ & 5.281831 & -1.463269 & -0.363953 & 2.23 & 0.462967 & $4.85 \times 10^{-24}$ \\
Fake pulsar 6 & 148.7190257 & $-6.73 \times 10^{-9}$ & 6.261385 & -1.141840 & 0.470985 & 0.97 & -0.153733 & $6.92 \times 10^{-25}$ \\
Fake pulsar 7 & 1220.979581 & $-1.12 \times 10^{-9}$ & 3.899513 & -0.356931 & 0.512323 & 5.25 & 0.756814 & $2.20 \times 10^{-24}$ \\
Fake pulsar 8 & 194.3083185 & $-8.65 \times 10^{-9}$ & 6.132905 & -0.583263 & 0.170471 & 5.89 & 0.073903 & $1.59 \times 10^{-23}$ \\
Fake pulsar 9 & 763.8473165 & $-1.45 \times 10^{-17}$ & 3.471208 & 1.321033 & -0.008560 & 1.01 & -0.619187 & $8.13 \times 10^{-25}$ \\
\hline \hline
\end{tabular}



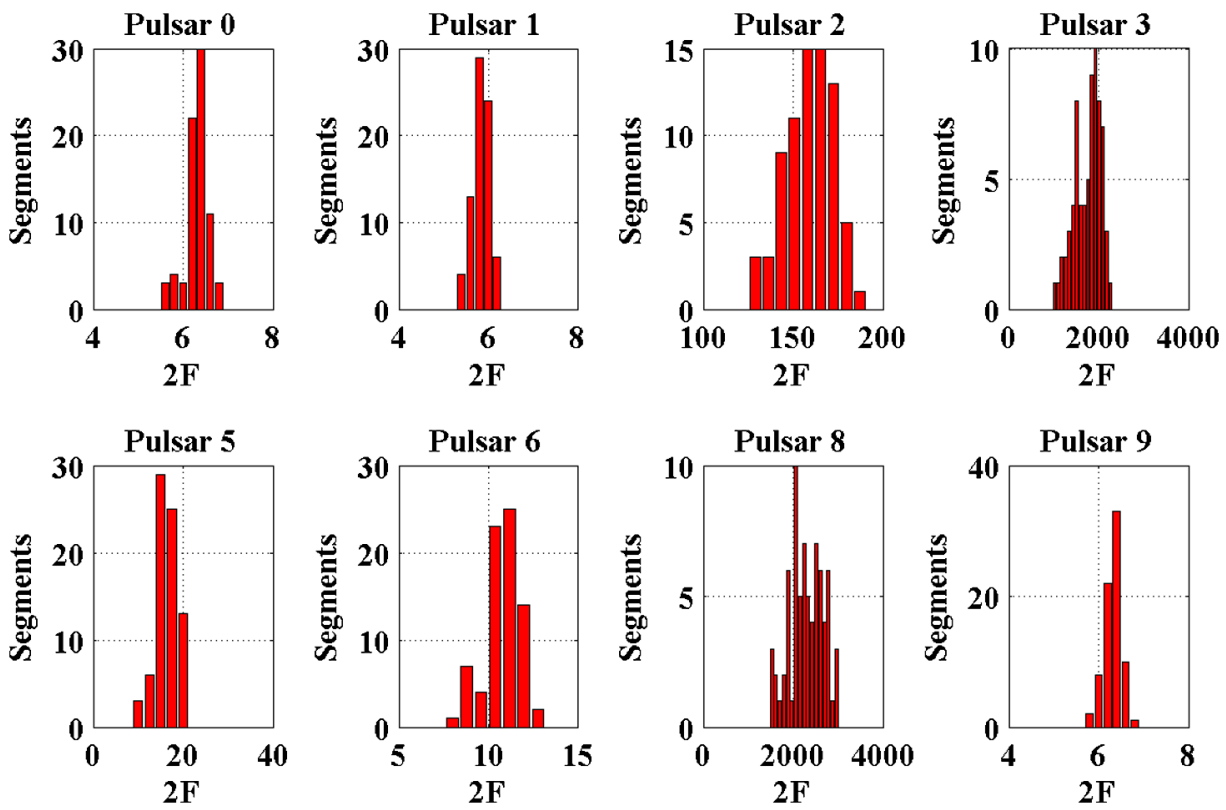

FIG. 11 (color online). Histograms of the $2 \mathcal{F}$ values estimated, for every data segment, for all the S5 hardware injections covered by the investigated frequency range. The largest $2 \mathcal{F}$ values are from the fake pulsars $2,3,5$, and 8 . In all the plots, the total number of data segments where the hardware injections were active is 76 .

data segments, and they were completely absent in the remaining 45 segments. Their expected value of the Hough number count is thus

$$
E\left[n_{\mathrm{c}}^{\mathrm{HI}}\right]=\sum_{j} \eta_{j} w_{j}+p \sum_{l} w_{l}
$$

where the superscript "HI" refers to Hardware Injection, $j$ runs over the number of data segments where the hardware injections were active and $l$ runs over the remaining segments, and the $w_{j}$ are the Hough weights given by Eq. (18); $\eta_{j}$ and $p$ are the probabilities that the estimated value of $2 \mathcal{F}$ (for a given data segment) crosses the threshold $2 \mathcal{F}_{\text {th }}=5.2$ in the presence and absence of a signal, respectively (expressions for $\eta_{j}$ and $p$ can be found in Refs. [12,19]).

Figure 11 shows, for each pulsar hardware injection, the histograms of the expected $2 \mathcal{F}$ values, computed for all the segments where a particular hardware injection was active. From this figure one can infer that the fake pulsars expected to be the loudest in terms of $2 \mathcal{F}$ values are pulsars 2 , 3,5 , and 8 .

The pulsar hardware injections go through the normal search and postprocessing pipelines in the usual way as described in the previous sections. As discussed in the following, the fake pulsars recovered in the S5R5 search are pulsars 2, 3, and 5. On the other hand, fake pulsars 6 and 8 are missed because their spin-down values are outside our search range. As expected, fake pulsars 0,1 , and 9 are not recovered because their amplitudes are too weak and they do not pass the $\left\langle 2 \mathcal{F}_{\mathrm{HL}}\right\rangle>6.5$ cut, as shown in Table IV. In this table, the observed number counts of 69 72 are consistent with noise fluctuations in those half- $\mathrm{Hz}$ bands (see Fig. 3) superseding the weak injected signals. Note that the expected $2 \mathcal{F}$ values shown in Fig. 11 were computed assuming a search using exactly the correct signal parameters provided in Table III, while the observed $\left\langle 2 \mathcal{F}_{\mathrm{HL}}\right\rangle$ values in Table IV were obtained from our search which uses a grid of templates, so significant mismatch is to be expected. Table IV compares the expected and observed values of the number counts associated with the S5 hardware injections and the surviving S5R5 candidate events closest to them. As in Sec. IVE, the measure of

TABLE IV. Comparison between the expected and observed number counts ( $E\left[n_{\mathrm{c}}^{\mathrm{HI}}\right]$ and $N_{\mathrm{c}}^{\mathrm{S} 5 \mathrm{R} 5}$, respectively) associated with the hardware injections and the recovered S5R5 candidates closest to these injections. The $\left\langle 2 \mathcal{F}_{\mathrm{HL}}\right\rangle$ values for each of the candidates are also listed. The fake signals labeled pulsar 4 and 7 were not taken into account in this analysis since they have frequencies outside the S5R5 search frequency range. The "expected" values marked by asterisks are not actually expected to be obtained because the spin-down rates for those signals lie outside the range of this search.

\begin{tabular}{lccr}
\hline \hline Name & $E\left[n_{\mathrm{c}}^{\mathrm{HI}}\right]$ & $N_{\mathrm{c}}^{\mathrm{S} 5 \mathrm{R} 5}$ & $\left\langle 2 \mathcal{F}_{\mathrm{HL}}\right\rangle$ \\
\hline Fake pulsar 0 & 53 & 69 & 5.6 \\
Fake pulsar 1 & 49 & 71 & 5.7 \\
Fake pulsar 2 & 88 & 80 & 22.3 \\
Fake pulsar 3 & 89 & 96 & 197.1 \\
Fake pulsar 5 & 85 & 70 & 6.6 \\
Fake pulsar 6 & $76^{*}$ & 71 & 5.3 \\
Fake pulsar 8 & $87^{*}$ & 72 & 5.5 \\
Fake pulsar 9 & 53 & 72 & 5.8 \\
\hline \hline
\end{tabular}


TABLE V. Study of hardware injections in the S5R5 search. Values of $(f, \alpha, \delta, \dot{f})$ for the fake pulsar 2, 3, 5 and for the closest recovered candidates (denoted as Cand $2,3,5)$ are listed. The $n_{c}$ value in the last column represents the expectation value $E\left[n_{\mathrm{c}}^{\mathrm{HI}}\right]$ for the fake pulsars and the observed number count $N_{\mathrm{c}}^{\mathrm{S} 5 \mathrm{R} 5}$ for the corresponding recovered candidate.

\begin{tabular}{lccccc}
\hline \hline Name & $f(\mathrm{~Hz})$ & $\alpha(\mathrm{rad})$ & $\delta(\mathrm{rad})$ & $\dot{f}\left(\mathrm{~Hz} \mathrm{~s}^{-1}\right)$ & $n_{\mathrm{c}}$ \\
\hline Fake pulsar 2 & 575.163573 & 3.756929 & 0.060109 & $-1.37 \times 10^{-13}$ & 88 \\
Cand 2 & 575.163556 & 3.757514 & 0.065354 & $-1.64 \times 10^{-11}$ & 80 \\
Fake pulsar 3 & 108.857159 & 3.113189 & -0.583579 & $-1.46 \times 10^{-17}$ & 89 \\
Cand 3 & 108.857158 & 3.09806 & -0.5839483 & $-1.64 \times 10^{-11}$ & 96 \\
Fake pulsar 5 & 52.8083243 & 5.281831 & -1.463269 & $-4.03 \times 10^{-18}$ & 85 \\
Cand 5 & 52.8082977 & 5.58845 & -1.470972 & $-1.64 \times 10^{-11}$ & 70 \\
\hline \hline
\end{tabular}

distance used here is a Euclidean distance, expressed in bins, in the four dimensions $(f, \dot{f}, \alpha, \delta)$.

Table $\mathrm{V}$ lists the parameters of pulsars 2,3 , and 5 and the parameters of the corresponding recovered candidates. We successfully find candidates near the correct signal parameters. The mismatch in spin down might seem large, but in fact the injections were found at the nearest spindown template. The number count values show consistency within the $3 \sigma$ range.

\section{CONCLUSIONS}

No evidence for continuous gravitational waves has been observed in the search presented here. Upper limits on the intrinsic gravitational wave strain have been derived using standard population-based methods and are shown in Fig. 9. These results are about a factor of 3 more constraining than those from the previous Einstein@ Home search in early S5 data [24]. This improvement comes from using more data (a year versus two months), from using a multidetector coherent statistic (versus a single-detector statistic), from a lower effective threshold in the coherent detection stage, and from a more sensitive incoherent method to combine the information from the coherently analyzed segments. The largest effect comes from lowering the effective threshold. Indeed much of the improvement in sensitivity is attributable to improved data analysis methods (as opposed to improved detector sensitivity). If we had used the much higher threshold of 25 on $2 \mathcal{F}$, as in Ref. [24], our sensitivity would have been a factor of $\sim 2.5$ worse than our final upper limits, ${ }^{7}$ thereby undoing almost all of the factor of 3 improvement mentioned above.

We have not included second time derivatives of the frequency in our search. This could be astrophysically significant in some regions of parameter space, as discussed in Sec. III D. It is important to keep this caveat in mind while interpreting our results.

This is the most sensitive wide-frequency-range, all-sky search for $\mathrm{CW}$ signals performed to date. The upper limit

\footnotetext{
${ }^{7}$ Note that this does not mean that simply lowering the threshold to 5.2 in the S5R1 search would increase the S5R1 sensitivity by a factor 2.5 .
}

values are comparable to those obtained recently using the PowerFlux method [22,26] on the entire S5 data set (S5R3 + S5R5). Reference [26] searched for CW signals over the whole sky, in a smaller frequency band (up to $800 \mathrm{~Hz}$ versus $1190 \mathrm{~Hz}$ here), but a broader spin-down range up to $-6 \mathrm{nHz} / \mathrm{s}$. Strain upper limits were set at the $95 \%$ confidence in $0.25-\mathrm{Hz}$ wide sub-bands. In particular, near $152 \mathrm{~Hz}$, the PowerFlux strict, all-sky upper limit on worst-case linearly (best-case circularly) polarized strain amplitude $h_{0}$ is $\sim 1 \times 10^{-24}\left(3.5 \times 10^{-25}\right)$. As a comparison, at the same frequency, this search constrains the strain to $h_{0} \leqslant 7.6 \times 10^{-25}$ (as shown in Fig. 9), $9.2 \times$ $10^{-25}$ and $3.2 \times 10^{-25}$ for the case of average, linear and circular polarization, respectively, with a $90 \%$ confidence level in a $0.5-\mathrm{Hz}$ wide band.

The most constraining upper limit obtained by this search is $h_{0}^{90 \%} \sim 7.6 \times 10^{-25}$ at $152 \mathrm{~Hz}$; the corresponding maximum reach is roughly $4 \mathrm{kpc}$, assuming $\varepsilon \sim 10^{-4}$.

It has long been expected that searching a large parameter space for $\mathrm{CW}$ signals will require hierarchical semicoherent searches. This analysis is a milestone towards that goal, and we expect that future analyses will build on the tools developed here.

There are a number of areas where further improvements are possible. In the latest round of analysis (an Einstein@Home processing run that began in March 2012), some of the postprocessing techniques developed for this analysis have been "moved upstream" to the hosts. One example is the generalized $\mathcal{F}$-statistic consistency test [56]. This continues the pattern of moving analyses formerly carried out in the postprocessing stage (for example, the incoherent combination step) onto host machines. Another step forward is in the semi-coherent algorithm that combines the coherent analyses from the different segments. The Hough algorithm described here turned out to be rather cumbersome, and does not combine the coarse and fine grids in an optimal way. The latest round of Einstein@Home processing makes use of a simpler optimal semicoherent method, which allows longer coherent time baselines to be used. This method, based on a detailed analysis of correlations in parameter space [57], is described in Ref. [58]. Looking farther forward, we expect to use higher-order spin-down parameters both to search 
for a broader class of signals as well as to be able to employ longer coherent time baselines in the analysis.

The Advanced LIGO and Advanced Virgo detectors are currently under construction, and should begin operations around 2016. In comparison with the current generation, these instruments will provide an order-of-magnitude improvement in strain sensitivity, increasing the volume of space observed by a factor of a thousand.

These and other improvements in data analysis methods and instrumentation make us optimistic that we will eventually be able to make direct detections of CW signals. Such detection will provide new insights into the internal structure, formation history and population statistics of neutron stars.

\section{ACKNOWLEDGMENTS}

The authors gratefully acknowledge the support of the United States National Science Foundation for the construction and operation of the LIGO Laboratory, the Science and Technology Facilities Council of the United Kingdom, the Max Planck Society, and the State of Niedersachsen/Germany for support of the construction and operation of the GEO600 detector, and the Italian Istituto Nazionale di Fisica Nucleare and the French Centre National de la Recherche Scientifique for the construction and operation of the Virgo detector. The authors also gratefully acknowledge the support of the research by these agencies and by the Einstein@Home volunteers, by the Australian Research Council, the International Science Linkages program of the Commonwealth of Australia, the Council of Scientific and Industrial Research of India, the Istituto Nazionale di Fisica Nucleare of Italy, the Spanish Ministerio de Economía y Competitividad, the Conselleria d'Economia Hisenda i Innovació of the Govern de les Illes Balears, the Foundation for Fundamental Research on Matter supported by the Netherlands Organisation for Scientific Research, the Polish Ministry of Science and Higher Education, the FOCUS Programme of Foundation for Polish Science, the Royal Society, the Scottish Funding Council, the Scottish Universities Physics Alliance, The National Aeronautics and Space Administration, the Carnegie Trust, the Leverhulme Trust, the David and Lucile Packard Foundation, the Research Corporation, and the Alfred P. Sloan Foundation. This document has been assigned LIGO Laboratory Document No. LIGOP1200026.

\section{APPENDIX A: PROBLEMS IN CALCULATING THE WEIGHTS}

In this section we describe two software errors that affected the main hierarchical search code. While these errors did not invalidate the search results and were dealt with adequately, we document them here for completeness.
The first issue is connected with the choice of weights used to construct the number count defined in Eqs. (17) and (18). The weights for each segment are computed following Eq. (19) which uses the harmonic mean of the noise spectra for each SFT. However, the original method for computing the weights used the arithmetic mean of the contributions instead of the harmonic mean, which turned out to have the perverse effect that segments with a few noisy SFTs got a disproportionately large weight. This led to a much larger value for the variance given in Eq. (22) and a correspondingly anomalously small value of the significance defined in Eq. (21). All WUs were originally run using the arithmetic mean which led to anomalously low values of the significance CR due to non-stationary noise for a small number of frequency bands: [50, 202], [328.5, 329], [995.5, 1010], and [1069, 1075] Hz. The WUs for these bands were re-computed with the weights given by Eq. (19). All other frequencies are unaffected by this problem.

A second issue, which interacts with the problematic calculation of the weights described above, is floating point inaccuracy in our implementation of the Hough-transform algorithm. A single threshold crossing for the $\mathcal{F}$-statistic leads to $\mathrm{a}+1$ in number count for possibly a large number of points in parameter space [18], and it is not necessary to step through parameter space point-by-point to calculate the final number count. For the vast majority of cases, our implementation of the Hough transform agrees with the brute force approach for calculating the number count, but the two can occasionally differ. If we were not using weights, these differences would have a minor effect on the number count. Occasionally, however, these floating point errors coincide with the cases when we assign excessively large weights to particular segments as discussed above. In these cases, the discrepancies in the number count can be large and in some cases may even exceed the number of segments, which is in principle a strict upper bound on the number count. Note that our upper limits remain valid because they consistently use the same search code, and any candidates are followed up by independent codes, thereby avoiding spurious false alarms. Using the modified weights based on Eq. (19) fixes this problem as well.

\section{APPENDIX B: S5R3 POSTPROCESSING}

The S5R3 run was launched on September 23, 2007, and ended on September 25, 2008. Like S5R5, it was an all-sky search. It used 7237 S5 LIGO SFTs, collected between the GPS times of 818845553 s (Sat Dec 17 09:05:40 GMT 2005) and 851765191 s (Tue Jan 0209:26:17 GMT 2007). The data analyzed consisted of 3781 SFTs from $\mathrm{H}$ and 3456 SFTs from $L$. The number of data segments used for the S5R3 run was 84, with duration each $T_{\text {seg }}=25$ hours. The search frequency range was $[50,1200] \mathrm{Hz}$, with a frequency resolution $\delta f \sim 6.7 \times 10^{-6} \mathrm{~Hz}$ and a mismatch 


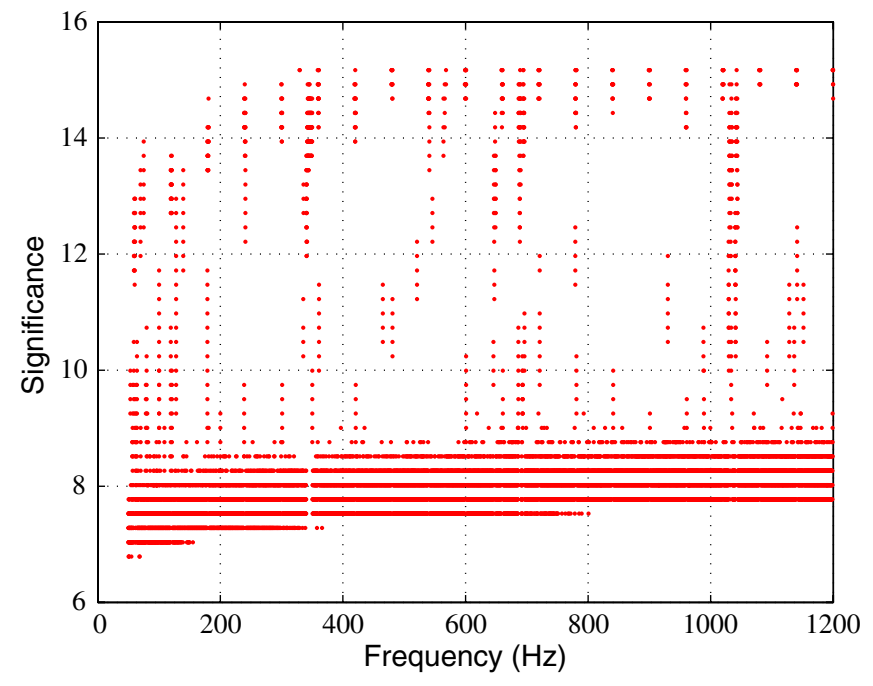

FIG. 12 (color online). Significance of 230000 loudest candidates selected in $0.5 \mathrm{~Hz}$-wide frequency bands as a function of the frequency.

value $m=0.3$, leading to the spin-down resolution given by Eq. (16). In the S5R3 run, each WU analyzed a constant frequency bandwidth $B \simeq 16 \mathrm{mH} z$, the full spin-down interval, ranging roughly from $-1.6 \mathrm{nHz} \mathrm{s}^{-1}$ to $-3.1 \times$ $10^{-11} \mathrm{~Hz} \mathrm{~s}^{-1}$ in steps of $3.8 \times 10^{-10} \mathrm{~Hz} \mathrm{~s}^{-1}$, and a certain region of the sky, as already discussed in Sec. III C. The sky grids and output file formats are identical to those used in S5R5. A major difference with S5R5 are the weights: no weighting scheme was used in S5R3, and all the weights $w_{i}$ appearing in Eq. (17) were set to unity. In total, $\sim 7 \times 10^{10}$ S5R3 candidates were sent back to the Einstein@Home server. The S5R3 postprocessing was performed before that for S5R5, and it was used as a "test-bed" for the latter. It consists of the same series of steps described previously in Sec. IV. The significance values of the 100 loudest candidates in $0.5 \mathrm{~Hz}$-wide frequency bands are plotted in Fig. 12 as a function of the frequency. These represent a total of 230000 candidates. This set is reduced by $\sim 27 \%$ via the removal of instrumental noise artifacts (listed in Tables VI and VII) and of the candidates from search frequency bands close to the fake noise (according to Table I). For all the surviving 167779 candidates, the $\mathcal{F}$-statistic consistency veto described in Sec. IVC removes an additional 3.6\%. Figure 13 shows the values of $\left\langle 2 \mathcal{F}_{\mathrm{H}}\right\rangle,\left\langle 2 \mathcal{F}_{\mathrm{L}}\right\rangle$ and $\left\langle 2 \mathcal{F}_{\mathrm{HL}}\right\rangle$ versus $\left\langle 2 \mathcal{F}_{\mathrm{HL}}\right\rangle$ for 161785 candidate events that survived (top plot) and 5994 that were excluded (bottom plot).

Candidates whose $\left\langle 2 \mathcal{F}_{\mathrm{HL}}\right\rangle$-value is greater than 6.5 , i.e., 1465 out of 161785 , are followed up by performing a hierarchical search using the S5R5 data set. This run, whose details are described in Sec. IV, consists of $\sim 46 \%$ more data than was used for S5R3 and is thus more sensitive than S5R5. The followed-up candidates are plotted in Fig. 14 as a function of the frequency: 87
TABLE VI. Known S5 H spectral artifacts. The different columns represent (I) the central frequency of the instrumental line; (II) the number of harmonics including the fundamental; (III) low-frequency (LF) bound of the knockout band; (IV) high-frequency (HF) bound of the knockout band; (V) the cause of the line (see key below). When there were higher harmonics, the third and fourth columns were multiplied by the harmonic number to yield the proper LF and HF bounds.

\begin{tabular}{|c|c|c|c|c|}
\hline$f_{\mathrm{L}}(\mathrm{Hz})$ & Harmonics & $\mathrm{LF}$ & $\mathrm{HF}$ & Cause \\
\hline 1.0 & 1000 & 0.9999194 & 1.0000806 & 6 Electronics \\
\hline 46.70 & 1 & 46.6932 & 46.7068 & Calibration \\
\hline 48 & 1 & 47.96 & 48.04 & Pulsed-heating \\
\hline 51 & 1 & 50.96 & 51.04 & Pulsed-heating \\
\hline 54 & 1 & 53.96 & 54.04 & Pulsed-heating \\
\hline 57 & 1 & 56.96 & 57.04 & Pulsed-heating \\
\hline 60 & 121 & 59.96 & 60.04 & Mains \\
\hline 63 & 1 & 62.96 & 63.04 & Pulsed-heating \\
\hline 66 & 1 & 65.96 & 66.04 & Pulsed-heating \\
\hline 69 & 1 & 68.96 & 69.04 & Pulsed-heating \\
\hline 72 & 1 & 71.96 & 72.04 & Pulsed-heating \\
\hline 85.80 & 1 & 85.79 & 85.81 & Electronics \\
\hline 89.9 & 1 & 89.84 & 89.96 & Electronics \\
\hline 93.05 & 1 & 93.04 & 93.06 & Unknown \\
\hline 93.25 & 1 & 93.24 & 93.26 & Unknown \\
\hline 139.95 & 1 & 139.94 & 139.96 & Electronics \\
\hline 164.52 & 1 & 164.51 & 164.53 & Electronics \\
\hline 329.51 & 2 & 329.49 & 329.53 & Wire \\
\hline 329.58 & 1 & 329.56 & 329.59 & Electronics \\
\hline 329.59 & 2 & 329.57 & 329.61 & Wire \\
\hline 329.70 & 2 & 329.67 & 329.72 & Wire \\
\hline 329.78 & 2 & 329.75 & 329.8 & Wire \\
\hline 329.86 & 1 & 329.85 & 329.87 & Electronics \\
\hline 335.695 & 1 & 335.67 & 335.72 & Wire \\
\hline 335.7230 & 1 & 335.698 & 335.748 & Wire \\
\hline 335.7410 & 1 & 335.716 & 335.766 & Wire \\
\hline 335.8200 & 1 & 335.795 & 335.845 & Wire \\
\hline 343.2879 & 1 & 343.261 & 343.315 & Wire \\
\hline 343.4145 & 1 & 343.394 & 343.435 & Wire \\
\hline 343.9272 & 1 & 343.907 & 343.948 & Wire \\
\hline 344.0584 & 1 & 344.038 & 344.079 & Wire \\
\hline 344.5247 & 1 & 344.499 & 344.55 & Wire \\
\hline 344.6685 & 1 & 344.647 & 344.69 & Wire \\
\hline 344.7186 & 1 & 344.692 & 344.745 & Wire \\
\hline 344.8280 & 1 & 344.810 & 344.847 & Wire \\
\hline 347.1824 & 1 & 347.16 & 347.204 & Wire \\
\hline 347.3107 & 1 & 347.29 & 347.331 & Wire \\
\hline 347.3635 & 1 & 347.34 & 347.387 & Wire \\
\hline 347.5099 & 1 & 347.489 & 347.531 & Wire \\
\hline 347.5818 & 1 & 347.557 & 347.606 & Wire \\
\hline 347.6860 & 1 & 347.664 & 347.708 & Wire \\
\hline 347.7230 & 1 & 347.703 & 347.743 & Wire \\
\hline 393.1000 & 1 & 393.093 & 393.107 & Calibration \\
\hline 539.43 & 1 & 539.42 & 539.44 & Electronics \\
\hline 546.06 & 3 & 545.89 & 546.21 & Wire \\
\hline 548.36 & 1 & 548.37 & 548.37 & Electronics \\
\hline
\end{tabular}


TABLE VI. (Continued)

\begin{tabular}{|c|c|c|c|c|}
\hline$f_{\mathrm{L}}(\mathrm{Hz})$ & Harmonics & $\mathrm{LF}$ & $\mathrm{HF}$ & Cause \\
\hline 564.07 & 3 & 563.9 & 564.22 & Wire \\
\hline 566.10 & 3 & 565.93 & 566.25 & Wire \\
\hline 568.10 & 3 & 567.93 & 568.25 & Wire \\
\hline 646.385 & 3 & 646.22 & 646.535 & Wire \\
\hline 648.835 & 3 & 648.67 & 648.985 & Wire \\
\hline 649.87 & 1 & 649.86 & 649.88 & Unknown \\
\hline 659.31 & 1 & 659.30 & 659.32 & Electronics \\
\hline 686.6615 & 1 & 686.634 & 686.689 & Wire \\
\hline 686.9176 & 1 & 686.896 & 686.939 & Wire \\
\hline 688.0224 & 1 & 688 & 688.044 & Wire \\
\hline 688.2825 & 1 & 688.26 & 688.305 & Wire \\
\hline 689.1301 & 1 & 689.108 & 689.152 & Wire \\
\hline 689.4262 & 1 & 689.404 & 689.449 & Wire \\
\hline 689.5036 & 1 & 689.482 & 689.525 & Wire \\
\hline 689.7361 & 1 & 689.715 & 689.758 & Wire \\
\hline 694.4720 & 1 & 694.447 & 694.497 & Wire \\
\hline 694.7292 & 1 & 694.703 & 694.755 & Wire \\
\hline 695.0220 & 1 & 694.999 & 695.045 & Wire \\
\hline 695.2091 & 1 & 695.185 & 695.233 & Wire \\
\hline 695.4274 & 1 & 695.404 & 695.451 & Wire \\
\hline 695.4814 & 1 & 695.457 & 695.506 & Wire \\
\hline 915.80 & 1 & 915.79 & 915.81 & Electronics \\
\hline 960 & 1 & 959.99 & 960.01 & Timing \\
\hline 961 & 1 & 960.99 & 961.01 & Timing \\
\hline 995.50 & 1 & 995.49 & 995.51 & Electronics \\
\hline 1009.70 & 1 & 1009.69 & 1009.71 & Electronics \\
\hline 1030.55 & 1 & 1030.48 & 1030.63 & Wire \\
\hline 1032.19 & 1 & 1032.16 & 1032.23 & Wire \\
\hline 1032.58 & 1 & 1032.56 & 1032.61 & Wire \\
\hline 1033.78 & 1 & 1033.77 & 1033.79 & Electronics \\
\hline 1033.8766 & 1 & 1033.84 & 1033.92 & Wire \\
\hline 1034.3294 & 1 & 1034.3 & 1034.36 & Wire \\
\hline 1034.4549 & 1 & 1034.42 & 1034.49 & Wire \\
\hline 1034.821 & 1 & 1034.78 & 1034.86 & Wire \\
\hline 1042.25 & 1 & 1042.18 & 1042.32 & Wire \\
\hline 1042.3785 & 1 & 1042.35 & 1042.41 & Wire \\
\hline 1042.8179 & 1 & 1042.8 & 1042.84 & Wire \\
\hline 1043.0272 & 1 & 1042.99 & 1043.06 & Wire \\
\hline 1043.3351 & 1 & 1043.31 & 1043.36 & Wire \\
\hline 1043.455 & 1 & 1043.38 & 1043.53 & Wire \\
\hline 1144.3 & 1 & 1144.29 & 1144.31 & Calibration \\
\hline 1374.4509 & 1 & 1374.43 & 1374.47 & Wire \\
\hline 1376.6139 & 1 & 1376.59 & 1376.64 & Wire \\
\hline 1377.1423 & 1 & 1377.12 & 1377.17 & Wire \\
\hline 1378.7493 & 1 & 1378.72 & 1378.78 & Wire \\
\hline 1379.3999 & 1 & 1379.37 & 1379.43 & Wire \\
\hline 1379.5062 & 1 & 1379.48 & 1379.53 & Wire \\
\hline 1380.0283 & 1 & 1380 & 1380.05 & Wire \\
\hline 1390.0061 & 1 & 1389.98 & 1390.03 & Wire \\
\hline 1390.6821 & 1 & 1390.66 & 1390.71 & Wire \\
\hline 1391.4240 & 1 & 1391.4 & 1391.45 & Wire \\
\hline 1391.5967 & 1 & 1391.57 & 1391.62 & Wire \\
\hline
\end{tabular}

TABLE VI. (Continued)

\begin{tabular}{llllll}
\hline \hline$f_{\mathrm{L}}(\mathrm{Hz})$ & Harmonics & LF & \multicolumn{1}{c}{ HF } & Cause \\
\hline 1718.5697 & 1 & 1718.54 & 1718.6 & Wire \\
1721.9155 & 1 & 1721.89 & 1721.94 & Wire \\
1724.0104 & 1 & 1723.94 & 1724.08 & Wire \\
1724.9704 & 1 & 1724.95 & 1725 & Wire \\
1725.6181 & 1 & 1725.59 & 1725.64 & Wire \\
1737.9391 & 1 & 1737.92 & 1737.96 & Wire \\
1738.9907 & 1 & 1738.97 & 1739.01 & Wire \\
1739.8250 & 1 & 1739.8 & 1739.85 & Wire \\
1740.0280 & 1 & 1740 & 1740.05 & Wire \\
\hline \hline
\end{tabular}

TABLE VII. Known S5 L spectral artifacts. The columns are the same as in Table VI.

\begin{tabular}{|c|c|c|c|c|}
\hline$\underline{f_{\mathrm{L}}(\mathrm{Hz})}$ & Harmonics & $\mathrm{LF}$ & $\mathrm{HF}$ & Cause \\
\hline 1.0 & 1000 & 0.9999194 & 1.0000806 & Electronics \\
\hline 54.7000 & 1 & 54.6932 & 54.7068 & Calibration \\
\hline 59.0683 & 1 & 58.9749 & 59.1617 & Pulsed-heating \\
\hline 59.3918 & 1 & 59.3146 & 59.469 & Pulsed-heating \\
\hline 59.7382 & 1 & 59.5942 & 59.8822 & Pulsed-heating \\
\hline 60 & 121 & 59.96 & 60.04 & Mains \\
\hline 60.2731 & 1 & 60.1556 & 60.3906 & Pulsed-heating \\
\hline 60.5918 & 1 & 60.5284 & 60.6552 & Pulsed-heating \\
\hline 60.9497 & 1 & 60.8609 & 61.0385 & Pulsed-heating \\
\hline 93.2903 & 1 & 93.2758 & 93.3048 & Electronics \\
\hline 96.7082 & 1 & 96.6959 & 96.7205 & Electronics \\
\hline 139.9387 & 1 & 139.92 & 139.958 & Electronics \\
\hline 145.0622 & 1 & 145.047 & 145.078 & Electronics \\
\hline 186.5874 & 1 & 186.565 & 186.61 & Electronics \\
\hline 193.4164 & 1 & 193.395 & 193.437 & Electronics \\
\hline 233.2314 & 1 & 233.185 & 233.277 & Electronics \\
\hline 241.7774 & 1 & 241.713 & 241.842 & Electronics \\
\hline 329.2339 & 2 & 329.216 & 329.252 & Wire \\
\hline 329.3409 & 2 & 329.323 & 329.359 & Wire \\
\hline 329.4025 & 2 & 329.379 & 329.426 & Wire \\
\hline 335.276 & 1 & 335.256 & 335.296 & Wire \\
\hline 335.4100 & 1 & 335.386 & 335.434 & Wire \\
\hline 335.5950 & 1 & 335.57 & 335.62 & Wire \\
\hline 335.7770 & 1 & 335.752 & 335.802 & Wire \\
\hline 342.9424 & 1 & 342.915 & 342.97 & Wire \\
\hline 343.0980 & 1 & 343.075 & 343.121 & Wire \\
\hline 343.355 & 1 & 343.335 & 343.375 & Wire \\
\hline 343.4726 & 1 & 343.451 & 343.494 & Wire \\
\hline 343.6231 & 1 & 343.6 & 343.647 & Wire \\
\hline 344.266 & 1 & 344.246 & 344.286 & Wire \\
\hline 344.4132 & 1 & 344.392 & 344.434 & Wire \\
\hline 346.6349 & 1 & 346.603 & 346.667 & Wire \\
\hline 346.8060 & 1 & 346.784 & 346.828 & Wire \\
\hline 346.8727 & 1 & 346.85 & 346.896 & Wire \\
\hline 346.9151 & 1 & 346.895 & 346.935 & Wire \\
\hline
\end{tabular}


TABLE VII. (Continued)

\begin{tabular}{|c|c|c|c|c|}
\hline$\underline{f_{\mathrm{L}}(\mathrm{Hz})}$ & Harmonics & $\mathrm{LF}$ & $\mathrm{HF}$ & Cause \\
\hline 346.9650 & 1 & 346.945 & 346.985 & Wire \\
\hline 347.0370 & 1 & 347.017 & 347.057 & Wire \\
\hline 396.7 & 1 & 396.693 & 396.707 & Calibration \\
\hline 685.9147 & 1 & 685.893 & 685.937 & Wire \\
\hline 686.2051 & 1 & 686.172 & 686.238 & Wire \\
\hline 686.8158 & 1 & 686.792 & 686.84 & Wire \\
\hline 687.0511 & 1 & 687.021 & 687.081 & Wire \\
\hline 687.3246 & 1 & 687.301 & 687.348 & Wire \\
\hline 688.8577 & 1 & 688.832 & 688.883 & Wire \\
\hline 693.4187 & 1 & 693.392 & 693.445 & Wire \\
\hline 693.6827 & 1 & 693.652 & 693.713 & Wire \\
\hline 693.7638 & 1 & 693.74 & 693.788 & Wire \\
\hline 693.9111 & 1 & 693.888 & 693.934 & Wire \\
\hline 693.9834 & 1 & 693.958 & 694.008 & Wire \\
\hline 694.0889 & 1 & 694.058 & 694.12 & Wire \\
\hline 960 & 1 & 959.99 & 960.01 & Timing \\
\hline 961 & 1 & 960.99 & 961.01 & Timing \\
\hline 1029.5578 & 1 & 1029.53 & 1029.58 & Wire \\
\hline 1030.7536 & 1 & 1030.73 & 1030.78 & Wire \\
\hline 1031.1536 & 1 & 1031.13 & 1031.18 & Wire \\
\hline 1033.5104 & 1 & 1033.49 & 1033.53 & Wire \\
\hline 1040.3507 & 1 & 1040.33 & 1040.37 & Wire \\
\hline 1040.6940 & 1 & 1040.67 & 1040.72 & Wire \\
\hline 1040.7343 & 1 & 1040.71 & 1040.76 & Wire \\
\hline 1040.7859 & 1 & 1040.76 & 1040.81 & Wire \\
\hline 1041.0204 & 1 & 1041 & 1041.04 & Wire \\
\hline 1041.1701 & 1 & 1041.15 & 1041.19 & Wire \\
\hline 1041.2731 & 1 & 1041.25 & 1041.29 & Wire \\
\hline 1150.0661 & 1 & 1149.15 & 1150.98 & Calibration \\
\hline 1151.9118 & 1 & 1151.56 & 1152.26 & Calibration \\
\hline 1372.9742 & 1 & 1372.95 & 1373 & Wire \\
\hline 1374.6601 & 1 & 1374.64 & 1374.68 & Wire \\
\hline 1375.2021 & 1 & 1375.18 & 1375.23 & Wire \\
\hline 1378.3695 & 1 & 1378.34 & 1378.39 & Wire \\
\hline 1387.3946 & 1 & 1387.37 & 1387.42 & Wire \\
\hline 1387.9327 & 1 & 1387.9 & 1387.96 & Wire \\
\hline 1387.9660 & 1 & 1387.92 & 1388.01 & Wire \\
\hline 1388.0561 & 1 & 1388.03 & 1388.08 & Wire \\
\hline 1388.3850 & 1 & 1388.35 & 1388.42 & Wire \\
\hline 1388.5530 & 1 & 1388.53 & 1388.58 & Wire \\
\hline 1388.7127 & 1 & 1388.69 & 1388.74 & Wire \\
\hline 1716.8006 & 1 & 1716.77 & 1716.83 & Wire \\
\hline 1718.8679 & 1 & 1718.84 & 1718.89 & Wire \\
\hline 1719.5480 & 1 & 1719.52 & 1719.57 & Wire \\
\hline 1723.4861 & 1 & 1723.46 & 1723.51 & Wire \\
\hline 1734.7999 & 1 & 1734.78 & 1734.82 & Wire \\
\hline 1735.9610 & 1 & 1735.94 & 1735.99 & Wire \\
\hline 1736.1977 & 1 & 1736.16 & 1736.23 & Wire \\
\hline 1736.4134 & 1 & 1736.39 & 1736.44 & Wire \\
\hline 1920.0000 & 1 & 1919.99 & 1920.01 & Timing \\
\hline 1921.0000 & 1 & 1920.99 & 1921.01 & Timing \\
\hline 1922.0009 & 1 & 1921.98 & 1922.02 & Timing \\
\hline
\end{tabular}
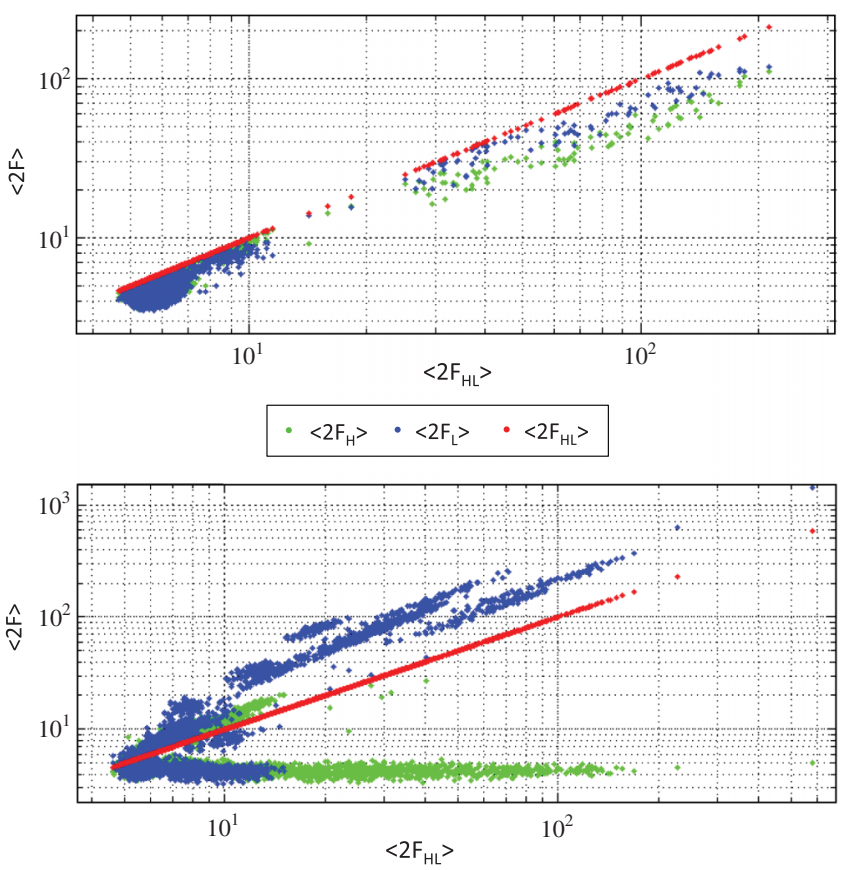

FIG. 13 (color). Values of $2 \mathcal{F}$ averaged over 84 data segments for the single-detector case, $\left\langle 2 \mathcal{F}_{\mathrm{H}}\right\rangle$ (green dots), $\left\langle 2 \mathcal{F}_{\mathrm{L}}\right\rangle$ (blue dots), the multidetector case $\left(\left\langle 2 \mathcal{F}_{\mathrm{HL}}\right\rangle\right.$, red dots) against those for the combined multidetector statistic $\left(\left\langle 2 \mathcal{F}_{\mathrm{HL}}\right\rangle\right)$. The top (bottom) plot shows such values for 161785 (5994) surviving (vetoed) candidates such that $\left\langle 2 \mathcal{F}_{\mathrm{H}}\right\rangle$ and (or) $\left\langle 2 \mathcal{F}_{\mathrm{L}}\right\rangle$ is less (greater) than $\left\langle 2 \mathcal{F}_{\mathrm{HL}}\right\rangle$.

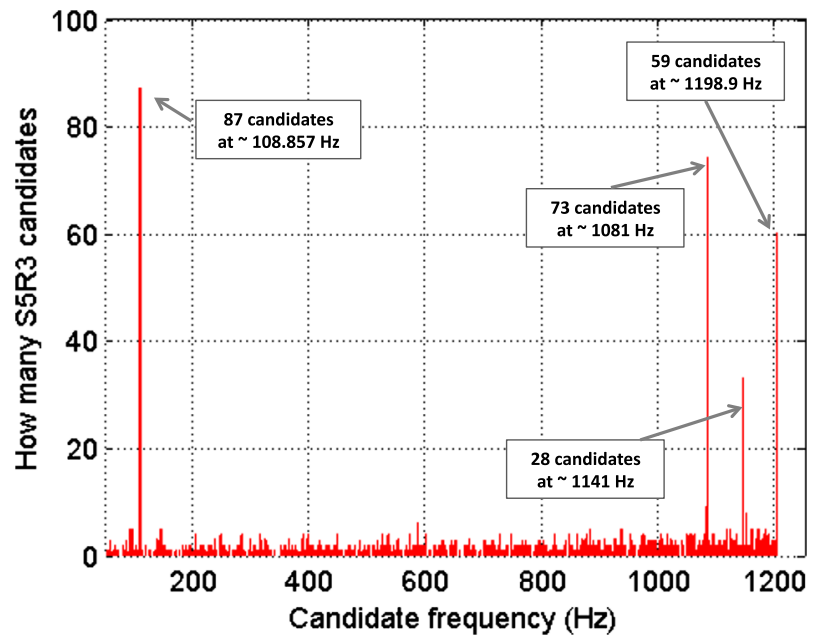

FIG. 14 (color online). Histogram of 1465 S5R3 candidates that have been further investigated through follow-up study using the S5R5 data set.

candidates are clustered at $\sim 108.857 \mathrm{~Hz}$ and represent the contribution of the hardware injection pulsar 3 [59]; 73 candidates have frequencies peaked around $\sim 1081 \mathrm{~Hz}$, 59 candidates at $\sim 1198.9 \mathrm{~Hz}$ and 28 at $\sim 1141 \mathrm{~Hz}$. Except for the candidates that represent the contribution from the hardware injected signal, none of the S5R5 
candidates is consistent with a signal at a level consistent with the observed S5R3 excess. We then conclude that no gravitational wave signal is observed in the S5R3 data.

\section{APPENDIX C: INSTRUMENTAL NOISE ARTIFACTS}

This appendix contains lists of the main known spectral lines of instrumental origin in the LIGO detectors during the S5 run. They were individually identified with a particular source, or were members of identified combs of lines found in many channels (e.g., $60 \mathrm{~Hz}$ combs), or the source was unknown, but they were found in frequency coincidence between the gravitational wave channel and auxiliary channels. In the latter case, to ensure that actual gravitational wave signals were not rejected, the SNR in the auxiliary channel had to be at least five times larger than in the gravitational wave channel and the density of lines in the auxiliary channel had to be low enough that accidental coincidence with a line in the gravitational wave channel was highly unlikely. The spectral lines and harmonics detected in $\mathrm{H}$ and $\mathrm{L}$ are listed in Tables VI and VII, respectively. As mentioned earlier, on the basis of these tables, about 22 and $25 \%$ of candidates have been excluded from the analysis in the S5R5 and S5R3 postprocessing, respectively.

For each candidate with frequency $f_{\mathrm{c}}$ and derivative $\dot{f}_{\mathrm{c}}$, the candidate was rejected if a band $\Delta f_{\mathrm{c}} \simeq f_{\mathrm{c}} \times 10^{-4}+$ $\left|\dot{f}_{\mathrm{c}}\right| \times 10^{7} \mathrm{~s}$ on either side of the signal had any overlap with an instrumental line band. This was done in order to take into account the maximum possible Doppler shift due to the Earth's orbital velocity, which is roughly $10^{-4}$ in units of the speed of light, and the maximum frequency shift due to the spin down of the source over the $\sim \pm 10^{7} \mathrm{~s}$ time span relative to the reference time during the Einstein@Home run.

A short explanation of the key to the line sources listed in the fifth column of Tables VI and VII follows.

Mains lines at multiples of the $60 \mathrm{~Hz}$ electrical power system frequency; the dominant coupling mechanism at $60 \mathrm{~Hz}$ was from magnetic fields generated by electric currents coupling to the permanent magnets mounted on the test masses.

Electronics produced by either electronic circuit oscillations or by slight data corruption associated with repetitive processes in the data acquisition computers. The line was identified in power supply voltage variation, magnetic fields from electronics or by direct measurements.

Calibration produced for calibration purposes by moving a test mass with the actuation system.

Timing introduced by the timing verification system.

Wire a vibrational resonance of a mirror suspension wire.

Pulsed-heating produced by cyclically pulsed mains heating circuits, coupling to the test mass magnets via magnetic fields.

Unknown a line of unknown source that appeared in auxiliary channels and met the rejection criteria noted in the text.
[1] L. Bildsten, Astrophys. J. 501, L89 (1998).

[2] G. Ushomirsky, C. Cutler, and L. Bildsten, Mon. Not. R. Astron. Soc. 319, 902 (2000).

[3] C. Cutler, Phys. Rev. D 66, 084025 (2002).

[4] A. Melatos and D. Payne, Astrophys. J. 623, 1044 (2005).

[5] B. J. Owen, Phys. Rev. Lett. 95, 211101 (2005).

[6] A. Abramovici, W. E. Althouse, R. W. Drever, Y. Gursel, S. Kawamura et al., Science 256, 325 (1992).

[7] B. P. Abbott et al., Rep. Prog. Phys. 72, 076901 (2009).

[8] C. Bradaschia, E. Calloni, M. Cobal, R. Del Fabbro, A. di Virgilio et al., in Proceedings of the Banff Summer Institute, Banff, Alberta, 1990, edited by R. Mann and P. Wesson (World Scientific, Singapore, 1991) p. 499.

[9] T. Accadia et al., JINST 7, P03012 (2012).

[10] K. Danzmann, in First Edoardo Amaldi Conference on Gravitational Wave Experiments, edited by E. Coccia, G. Pizzella, and F. Ronga (World Scientific, Singapore, 1995), p. 100.

[11] K. Tsubono, in First Edoardo Amaldi Conference on Gravitational Wave Experiments, edited by E. Coccia, G. Pizzella, and F. Ronga (World Scientific, Singapore, 1995) p. 100.
[12] P. Jaranowski, A. Królak, and B. F. Schutz, Phys. Rev. D 58, 063001 (1998).

[13] C. Cutler and B.F. Schutz, Phys. Rev. D 72, 063006 (2005).

[14] R. Prix, Phys. Rev. D 75, 023004 (2007); 75, 069901(E), 2007.

[15] R. Prix and B. Krishnan, Classical Quantum Gravity 26, 204013 (2009).

[16] B. Abbott et al. (LIGO Scientific Collaboration), Phys. Rev. D 76, 082001 (2007).

[17] J. Abadie et al. (LIGO Scientific Collaboration), Astrophys. J. 722, 1504 (2010).

[18] P. R. Brady and T. Creighton, Phys. Rev. D 61, 082001 (2000).

[19] B. Krishnan, A. M. Sintes, M. A. Papa, B. F. Schutz, S. Frasca, and C. Palomba, Phys. Rev. D 70, 082001 (2004).

[20] C. Cutler, I. Gholami, and B. Krishnan, Phys. Rev. D 72, 042004 (2005).

[21] B. Abbott et al. (LIGO Scientific Collaboration), Phys. Rev. D 72, 102004 (2005).

[22] B. Abbott et al. (LIGO Scientific Collaboration), Phys. Rev. D 77, 022001 (2008). 
[23] B. Abbott et al. (LIGO Scientific Collaboration), Phys. Rev. D 79, 022001 (2009).

[24] B. Abbott et al. (LIGO Scientific Collaboration), Phys. Rev. D 80, 042003 (2009).

[25] B. Abbott et al. (LIGO Scientific Collaboration), Phys. Rev. Lett. 102, 111102 (2009).

[26] J. Abadie et al. (LIGO Scientific Collaboration and Virgo Collaboration), Phys. Rev. D 85, 022001 (2012).

[27] B. Krishnan (LIGO Scientific Collaboration), Classical Quantum Gravity 22, S1265 (2005).

[28] Einstein@Home website, http://einstein.phys.uwm.edu.

[29] R. Smoluchowski, Phys. Rev. Lett. 24, 923 (1970).

[30] M. Ruderman, Astrophys. J. 382, 587 (1991).

[31] C. Horowitz and K. Kadau, Phys. Rev. Lett. 102, 191102 (2009).

[32] J. H. Taylor and J. Weisberg, Astrophys. J. 345, 434 (1989).

[33] S. Bonazzola and E. Gourgoulhon, Astron. Astrophys. 312, 675 (1996).

[34] R. Balasubramanian, B. Sathyaprakash, and S. Dhurandhar, Phys. Rev. D 53, 3033 (1996).

[35] B. J. Owen, Phys. Rev. D 53, 6749 (1996).

[36] D. M. Whitbeck, Ph.D. thesis, The Pennsylvania State University.

[37] H. J. Pletsch, Phys. Rev. D 82, 042002 (2010).

[38] P. R. Brady, T. Creighton, C. Cutler, and B.F. Schutz, Phys. Rev. D 57, 2101 (1998).

[39] C. Palomba, P. Astone, and S. Frasca, Classical Quantum Gravity 22, S1255 (2005).

[40] Boinc website, http://boinc.berkeley.edu/.

[41] D. P. Anderson, in Proceedings of the Fifth IEEE/ACM International Workshop on Grid Computing (GRID04) (IEEE Computer Society, Washington, DC, 2004), p. 4.
[42] D. P. Anderson, C. Christensen, and B. Allen, in Proceedings of the 2006 ACM/IEEE Conference on Supercomputing (IEEE Computer Society, Tampa, 2006), p. 126.

[43] J. Ostriker and J. Gunn, Astrophys. J. 157, 1395 (1969).

[44] A. G. Lyne, R. S. Pritchard, F. Graham-Smith, and F. Camilo, Nature (London) 381, 497 (1996).

[45] A. G. Lyne et al., Mon. Not. R. Astron. Soc. 265, 1003 (1993).

[46] C. Aulbert and H. Fehrmann, Max-Planck-Gesellschaft (2008), http://www.mpg.de/308429/forschungsSchwerpunkt.

[47] P. Leaci, M. A. Papa, and H. Pletsch (to be published).

[48] C. Audet and J. E. Dennis, Jr., SIAM J. Optim. 17, 188 (2006).

[49] S. Le Digabel, ACM Trans. Math. Softw. 37, 44:1 (2011).

[50] M. Shaltev, (http://arxiv.org/abs/1201.4656).

[51] M. A. Abramson, C. Audet, J.E. Dennis, Jr., and S. Le Digabel, SIAM J. Optim. 20, 948 (2009).

[52] See Supplemental Material at http://link.aps.org/ supplemental/10.1103/PhysRevD.87.042001 for numerical values of upper limits.

[53] J. Abadie et al. (LIGO Scientific Collaboration), Nucl. Instrum. Methods Phys. Res., Sect. A 624, 223 (2010).

[54] K. Wette, Phys. Rev. D 85, 042003 (2012).

[55] R. Prix and K. Wette, LIGO Report No. T1200272, 2012.

[56] R. Prix, D. Keitel, M. A. Papa, P. Leaci, and M. Siddiqi (unpublished).

[57] H. J. Pletsch, Phys. Rev. D 78, 102005 (2008).

[58] H. J. Pletsch and B. Allen, Phys. Rev. Lett. 103, 181102 (2009).

[59] P. Leaci (LIGO Scientific Collaboration and Virgo Collaboration), LSC and Virgo Report No. T1200278, 2012, https://dcc.ligo.org/cgi-bin/DocDB/ShowDocument ?docid $=92307$. 